NISTIR 7252

\title{
Particle Image Velocimetry Measurements of Buoyancy Induced Flow Through a Doorway
}

Rodney A. Bryant 
NISTIR 7252

\section{Particle Image Velocimetry Measurements of Buoyancy Induced Flow Through a Doorway}

Rodney A. Bryant Building and Fire Research Laboratory Fire Research Division

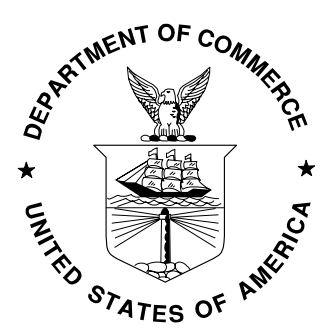

U.S. DEPARTMENT OF COMMERCE Carlos M. Gutierrez, Secretary TECHNOLOGY ADMINISTRATION Michelle O'Neill, Acting Under Secretary of Commerce for Technology NATIONAL INSTITUTE OF STANDARDS AND TECHNOLOGY William A. Jeffrey, Director 


\section{DISCLAIMER}

Certain commercial entities, equipment, or materials may be identified in this document in order to describe an experimental procedure or concept adequately. Such identification is not intended to imply recommendation or endorsement by the National Institute of Standards and Technology, nor is it intended to imply that the entities, materials, or equipment are necessarily the best available for the purpose. 


\begin{abstract}
Quantifying the ventilation available to an enclosure fire is an important step to understanding fire behavior. Accurate measurements of mass flow rate across an enclosure opening require a complete mapping of the velocity and density fields due to the three dimensional nature of vent flows. Conventional flow measurement methods in fire research consist of vertical arrays of thermocouples and differential pressure probes at the vent plane which are physically obtrusive and offer limited spatial sampling. A reduced-scale analog of a full-scale fire experiment was studied for the purpose of studying the potential use of Stereoscopic Particle Image Velocimetry, a laser based nonintrusive imaging technique, to measure fire induced flows through vents. The experiment was isothermal and modeled the convective transport by using a helium plume as the buoyant source. Stereoscopic PIV measurements were successfully demonstrated for a large-scale flow field with planar image regions of $0.71 \mathrm{~m} \mathrm{x} 0.62 \mathrm{~m}(1$ $\mathrm{x} h$ ). Measurements of the complete velocity vector, $v_{x}, v_{y}, v_{z}$, were performed and a full mapping of the velocity field in the region of the doorway was achieved. The vector field data displays the three dimensional structure of the flow through the doorway, revealing regions where the velocity component normal to the doorway plane may not completely dominate the velocity vector. A comparison of mass flow rate computations using the velocity component normal to the opening, $v_{x}$, and the velocity magnitude, demonstrated that mass flow rate will be over predicted by as much as $25 \%$ if computed using the velocity magnitude. Velocity magnitude is representative of bi-directional probe measurements and the over prediction is consistent with the use of doorway flow orifice coefficients to correct mass flow rates computed from bi-directional probe data. The intermediate scale of the flow field was sufficient to test the performance of a current PIV system and to identify the requirements for conducting successful PIV measurements in a full-scale fire experiment.
\end{abstract}




\section{ACKNOWLEDGEMENTS}

The success of any experimental project is due to more than just the work of the author. Therefore the author wishes to thank the following NIST staff for their valuable contributions: Marco Fernandez and Michael Selepak, NIST Fire Research Technicians, for fabrication of the reduced-scale enclosure and sampling probes, and Xiabo Yao, a University of Maryland graduate student in Fire Protection Engineering, for assisting with experimental setup. 


\section{TABLE OF CONTENTS}

page

ABSTRACT

III

ACKNOWLEDGEMENTS

IV

1 INTRODUCTION

2 FIRE INDUCED FLOW THROUGH VERTICAL VENTS

2.1 A SimPLE ILLUSTRATION

2.2 METHODS OF MEASUREMENT

2

3 PARTICLE IMAGE VELOCIMETRY

3.1 Two Dimensional PIV

3.2 STEREOSCOPIC PIV

3.3 LARGE-SCALE PIV

4 REDUCED-SCALE CONVECTIVE ANALOG EXPERIMENT

4.1 FIRE EXPERIMENT SCALING

4.2 EXPERIMENTAL SETUP

5 UNCERTAINTY ANALYSIS

5.1 TYPE A UNCERTAINTY

30

5.2 TYPE B UNCERTAINTY

30

5.3 UNCERTAINTY PROPAGATION

31

33

6 RESULTS OF REDUCED-SCALE EXPERIMENTS 35

6.1 VECTOR PROCESSING 35

6.2 FLOW VISUALIZATIONS

6.3 GAS DENSITY 49

6.4 MASS Flow Rate 51

7 CONCLUDING REMARKS

REFERENCES

$\begin{array}{ll}\text { APPENDIX } & 60\end{array}$

$.1 \quad$ TABULATED VALUES OF THE VELOCITY AND DENSITY FIELD AT THE DOORWAY PLANE

.2 NUMERICAL INTEGRATION 64 


\section{Introduction}

Fires release large amounts of energy. This energy, mostly in the form of heat, raises the temperature of the surrounding gases, causing them to expand. In the case of a fire within an enclosure, the hot gas will fill the top of an enclosure and increase the pressure within the enclosure, therefore causing gas to move out of the enclosure through the available openings. At the same time, the buoyant hot gases that rise to the top of the enclosure induce a low pressure region at the floor of an enclosure into which ambient gases will flow. The fire acts as a pump, pulling ambient air in while pushing combustion products mixed with air out. The openings which the gases flow through are referred to as vents and typically are doorways and windows. Quantifying the ventilation available to an enclosure fire is an important step to understanding fire behavior. The fresh air that moves into an enclosure provides the necessary oxygen to "feed" the fire while also serving to moderate the temperature of the compartment, depending on the stage of the fire growth. The hot fire gases that move out of an enclosure transfer heat and combustion products from the localized point of the fire to remote locations within a built structure. Ventilation therefore can play two important roles in fire spread, 1) to reduce the hazard by moderating the enclosure temperature and 2) to increase the hazard by transferring the heat, smoke and toxic gases to other locations of a built structure.

Measuring the velocity field across an opening together with the density field is necessary to quantify the mass flow rate of gas into and out of a burning room. Ideally this requires a full mapping of the velocity and density fields due to the three dimensional nature of vent flows. Early treatments quantifying fire induced flow through vents relied on Bernoulli's equation and a hydraulics-orifice approach $[1,2,3]$, therefore only a few well placed pressure and temperature measurements were required. Later treatments were improved by adding vertical arrays of pressure and temperature measurements and scanning them across the vents in order to address the three dimensional nature of the flows. $[4,5]$

Ventilation measurements have typically consisted of vertically spaced differentialpressure and temperature measurements between the enclosure and the ambient or within the vent plane itself. Emmons [6] presents a good review of the different levels of sophistication for these typical measurements. Velocity and gas density, both required to compute mass flow rate, are inferred from the pressure and temperature measurements. Velocity is coupled to gas density through the gas temperature measurement. These measurements are performed with physical probes which inherently alter the flow and are subject to the extreme conditions of a fire.

The typical instrument for velocity measurement in a fire test is the bi-directional probe, developed by McCaffrey and Heskestad. [7] The probe was designed to measure localized differential pressure in areas were the bulk flow direction can change. It is a robust device capable of withstanding the harsh conditions produced by fires. Some laser based techniques offer a non-intrusive and independent measurement of gas velocity. Single Point LDV (Laser Doppler Velocimetry) measurements of gas velocity in the 
doorway have been demonstrated in a fluid flow analog of an enclosure fire. [8] Single point measurements are insufficient for fire testing due to the need to map a vertical profile at minimum. A planar field laser based measurement such as Particle Image Velocimetry is a promising technique due to its potential ability to map the entire area of a vent opening without the need to translate any probes.

Basic PIV delivers a planar image of two components of the velocity vector, however more advanced systems offer all three components of the velocity vector. PIV has been employed with great success in small-scale flow fields. Applying the technique to flow fields typically encountered in fire systems presents a host of challenges, the minimum of which includes meeting the illumination and seeding requirements to ensure the accuracy of the measured velocity vectors. PIV systems have evolved into turnkey measurement devices but experimental setup and interpretation of the results is not entirely routine.

In order to create a better understanding of the challenges to be encountered when performing PIV measurements for full-scale fire tests, a reduced-scale model of a fire experiment was constructed. The reduced-scale experiment employed a helium plume as the buoyant source to produce an isothermal fluid-flow analog of the fire experiment. The experiment is a model of experiments by Steckler, Quintiere, and Rinkinen [9] which are possibly the most comprehensive doorway flow measurements to date. PIV and gas concentration measurements were conducted to measure the mass flow rate into and out of the enclosure doorway. The scale of the flows and the size of the measurement regions were intermediate, therefore they were not quite equivalent to what would be experienced for a full-scale fire test but large enough to push the performance envelope for current PIV systems. A major result of this study is the identification of the requirements for conducting successful PIV measurements in full-scale fire tests as well as laying the foundation for a measurement methodology.

\section{Fire Induced Flow Through Vertical Vents}

\subsection{A Simple Illustration}

Consider an enclosure with a single vertical opening, such as a doorway, and a fire source located on the floor and well within the enclosure. The fire produces a plume of hot gas that rises upward into the top of the enclosure due to buoyancy. The plume consists of combustion products and air that has mixed with the plume due to the entrainment along the edges. The hot gas fills the top of the enclosure, developing a layer that grows in depth and ultimately spills out of the top of the doorway, creating an outward flow of gas due to the increase in pressure within the enclosure. At the same time a flow of fresh air into the enclosure occurs in the bottom layer of the doorway. This flow of air is driven by a low pressure region which is induced by the lighter gas in the upper layer and the entrainment of air into the buoyant plume. An illustration of this model is shown in Figure 1 . The fire acts as a pump by pulling ambient air in while simultaneously pushing combustion products mixed with air out. 


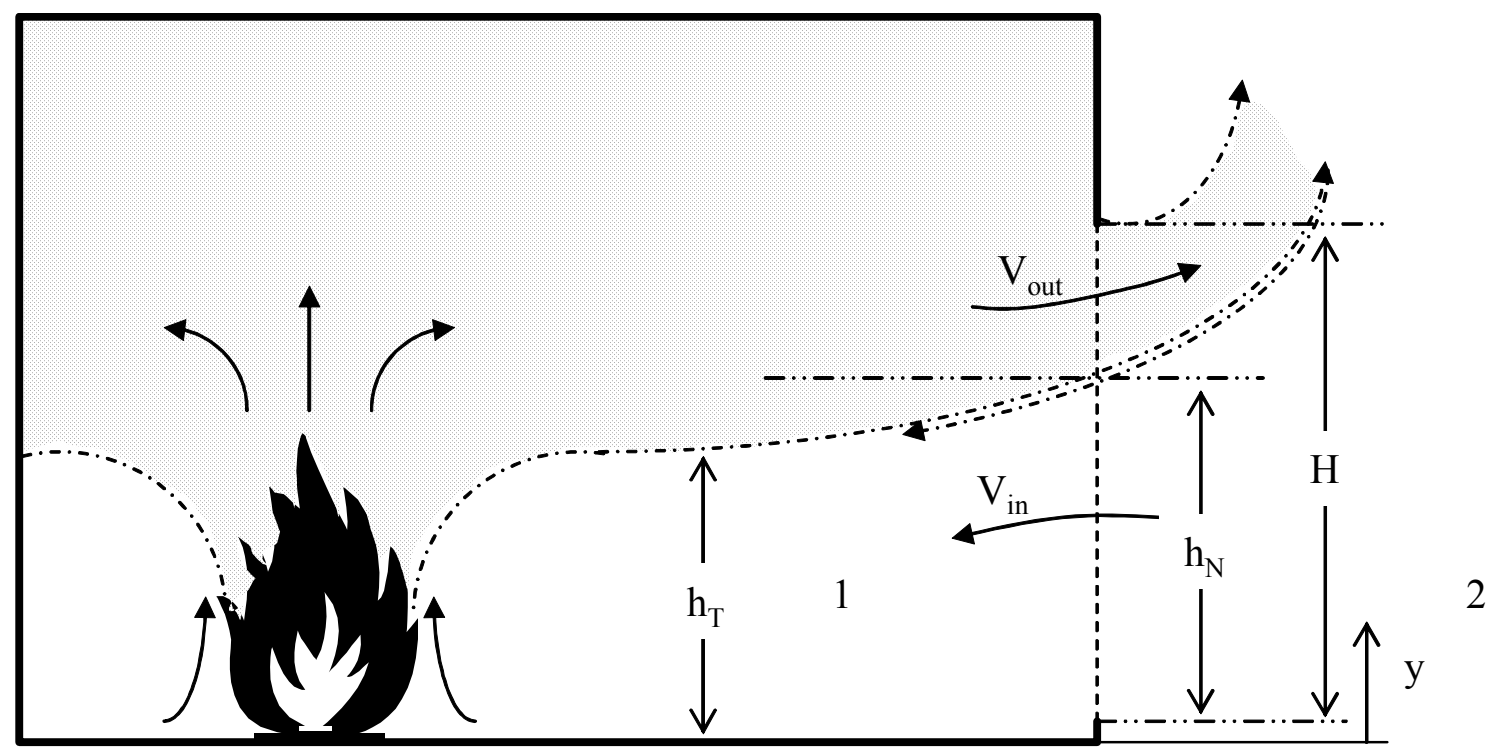

Figure 1 Fire induced flow for an enclosure with a single vertical vent opening.

Both the inflow and outflow occur within a single vent, creating a countercurrent flow. At the interface of the two flows the horizontal velocity component (parallel to the bulk flow direction) is zero. This interface is defined as the neutral plane with height, $h_{N}$, above the door sill. The local velocity in the doorway will depend upon the height above or below the neutral plane.

Within the enclosure two layers of gas exist, an upper layer of hot combustion products and air and a lower layer of cold air. The interface of these two layers is defined by the maximum vertical temperature gradient and has height, $h_{T}$. Similar to velocity there is a vertical distribution of temperature within the enclosure.

Time averaged mass flow rates of gas entering and exiting a vent are the typical parameters used to quantify ventilation in an enclosure fire. Theoretically the mass flow rate can be determined by integrating the product of local gas density, $\rho$, and velocity normal to the vent plane, $v_{x}$, across the inflow and outflow areas of the doorway. Equations 1 and 2 show that the gas density and velocity can vary with both vertical and horizontal location across the doorway. The height, $H$, and width, $W$, of the doorway along with the location of the neutral plane, $h_{N}$, establish the limits of integration. The $z$ coordinate is the horizontal direction normal to the plane of Figure 1, or parallel to the width of the door.

Theoretical mass flow rate in:

$$
\dot{m}_{i n, \text { thrtcl }}=\int_{-W / 2}^{W / 2} \int_{y_{o}}^{h_{N}} \rho(y, z) v_{x}(y, z) d y d z
$$

Theoretical mass flow rate out: 


$$
\dot{m}_{\text {out }, \text { thrtcl }}=\int_{-W / 2}^{W / 2} \int_{h_{N}}^{H} \rho(y, z) v_{x}(y, z) d y d z
$$

The general flow behavior indicated in Figure 1 can be derived from some visual observations of enclosure fires. However a more detailed description of the physical processes and their relationship to measurable quantities is required to define an ideal mass flow rate computation. Assuming a one-dimensional flow, this can be achieved with a hydrodynamics analysis. Kawagoe [10] developed such a hydrodynamic model to describe under-ventilated fires. The model was equally applicable to over-ventilated or developing fires and Rockett [2] presents the more generalized model for such applications. Emmons [6] has summarized the model and a similar summary is presented here to provide adequate background for the reader.

The model assumes the following for an enclosure with a single vertical vent as illustrated in Figure 1:

1) the entire system is at steady state

2) the gas inside the enclosure is a stratified layer of hot gas in the upper region and a layer of cool ambient gas in the lower region

3) a steep thermal gradient serves as the boundary between the two layers

In order for flow to occur across the vent a pressure difference must exist. Due to gravity, ambient pressure decreases with increasing height. In addition, inside the room, in the plane of the doorway, and just outside the room, the gas temperature and therefore the gas density varies with respect to height. Starting at the floor of the enclosure $(y=0)$ and assigning region 1 as inside the room and region 2 as outside the room the pressure difference is

$$
\Delta p_{f}=p_{f 1}-p_{f 2}
$$

where $p_{f 1}$ is the pressure at the floor inside the enclosure and $p_{f 2}$ is the ambient pressure at the floor level outside the enclosure.

The pressure difference at any height $y$ within the plane of the doorway can be determined from the following equations.

Inside the enclosure:

$$
p_{1}=p_{f 1}-\int_{0}^{y} \rho_{1} g d y
$$

Outside the enclosure: 


$$
p_{2}=p_{f 2}-\int_{0}^{y} \rho_{2} g d y
$$

The resulting pressure difference across the vent at any height, $h$, is then:

$$
\Delta p=p_{1}-p_{2}=\Delta p_{f}-\int_{0}^{h}\left(\rho_{2}-\rho_{1}\right) g d y
$$

Flow will be out of the enclosure for a positive pressure difference and into the enclosure for a negative pressure difference (relative to the sign convention applied in this study). The above equations give evidence to the pressure differential and associated gas flow across the doorway. Flow velocity has traditionally been inferred from pressure differential measurements. A brief summary of how pressure probe measurements have been applied to quantify fire ventilation will be provided in sections to follow.

\subsection{Methods of Measurement}

If it is assumed that points 1 and 2 lie along a streamline where the flow is steady, without friction and mass is conserved, then Bernoulli's equation describes the relation between the pressure difference and the flow velocity.

$$
p_{1}-p_{2}=\frac{\rho}{2}\left(V_{2}^{2}-V_{1}^{2}\right)
$$

Consider the gas flowing from the hot upper layer, region 1 Figure 1, through the top of the doorway and out into the ambient. Region 2 for this outflow is defined as the hot plume just outside the doorway If the assumption is made that the fluid accelerates from rest inside the enclosure, then $V_{1}=0$ and the flow out of the top of the doorway can be described by the familiar relation for flow velocity and pressure, Equation 8 . The same relation describes the flow into the bottom portion of the doorway with the assumption that the flow accelerates from rest in the ambient of region 2.

$$
\Delta p=\frac{\rho V^{2}}{2}
$$

Equation 8 demonstrates that it is possible to infer flow velocity across the doorway from a differential pressure measurement. Pitot-static probes have long been used to measure local differential pressure and therefore to infer local flow velocity. Because the probe is a physical obstruction to the flow the right hand side of the relationship as described in the equation above must be multiplied by a correction constant, $K$. The constant $K$ is a function of Reynolds number, $R e=V D / v$, where $D$ is the diameter of the probe and $v$ is the gas kinematic viscosity. $K$ is usually determined by calibration and will be close to unity for a well designed probe. 
Pitot-static probes measure flow velocity in a single direction and are therefore excellent instruments for experiments where the flow direction is known or the three dimensional motion of the flow is not significant. Fire induced vent flows, as shown in Figure 1, are counter-current flows, therefore flowing in opposite directions. To properly quantify a vent flow for a developing enclosure fire, measurements must be continuously performed at multiple locations along a vertical line. The location of the flow direction shift is only moderately predictable in the case of a steady state fire and for a developing fire the location will vary. In addition, flow in the upper layer and near the boundary of the vent may be highly three dimensional. The application of pitot-static probes to measure fire induced vent flows therefore has limitations. McCafferry and Heskestad [7] developed a modified pitot-static probe to address these limitations, the bi-directional probe.

Bi-directional probes were designed specifically to measure velocity in flow fields where the direction of the flow can change by 180 degrees. Their orientation within a flow is typically with the major axis of the probe parallel to the bulk flow direction. The angular sensitivity of bi-directional probes is $10 \%$ or less in the range of $(0-40)$ deg for off-axis flow. Bi-directional probes are less sensitive to flow direction compared to pitot-static probes due to the pressure taps being located within large flow capture wells. Sensitivity to flow direction is reduced as the size of the well is increased. However spatial resolution and velocity component selectivity is reduced due to the larger probe. A bidirectional probe essentially senses the flow speed without any off-axis directional information. Even though a bi-directional probe may be aligned with the bulk flow, the resulting measurement may not accurately represent the desired flow component if threedimensional motion is significant.

It is apparent from Equation 6 that the pressure differential in the doorway is height dependent. Therefore a vertical array of pressure probes is required to measure the velocity through the doorway. Each pressure probe requires a pressure transducer to detect the induced pressure differential. Because fire induced flows are very low speed $\mathrm{O}(10 \mathrm{~m} / \mathrm{s})$, each transducer must accurately resolve pressure differentials on the order of $10 \mathrm{~Pa}$.

Equation 9 demonstrates that measurements of pressure and density are required to infer the local velocity. Density is derived from a thermocouple measurement of temperature by applying the equation of state for an ideal gas and assuming a constant molecular weight.

$$
V=C \sqrt{\frac{2 \Delta p}{\rho}}
$$

It is possible to measure the local gas velocity through other methods such as Laser Doppler Velocimetry (LDV), Particle Tracking (PT) or Particle Image Velocimetry (PIV). Each of these methods requires that the flow is seeded with a tracer that follows the flow, typically tiny particles such as smokes or fogs. Velocity information is inferred from the measured Doppler frequency shift when LDV measurements are applied. These are typically single point measurements. Particle displacement is measured by particle 
tracking and PIV. These measurements are typically image measurements, therefore offering more spatial information. In addition, each of the three techniques is nonintrusive. It is acceptable to think of the seed particles as the probes; very tiny probes that move and react to the flow. In the case that the flow reacts to the presence of the seed particles, the particles are considered intrusive, and the seed loading would be too great for either of the techniques to work.

PIV, specifically stereoscopic PIV, is explored in this study to provide an independent measurement of local flow velocity in vent openings. Stereoscopic PIV measures all three components of the velocity vector therefore providing directional selectivity and information on the three dimensional flow dynamics. PIV measurements are inherently planar measurements with good spatial resolution, therefore field measurements are now possible. This offers orders of magnitude more measurements in a single acquisition than previously available and makes the calculation of mass flow rate described in Equations 1 and 2 a closer reality.

As beneficial as PIV is presented, it is not a trivial technique to apply. It requires specialty lasers, optics, digital cameras, and complex control and analysis software. These systems are not inexpensive, but market growth has lowered the cost while at the same time the performance has improved. PIV has been applied with great success in small laboratory-scale experiments, but large-scale flows such as those encountered in fire systems push the performance limits of PIV. Fortunately, the laser and digital camera industry have improved dramatically over the current decade in order to make such measurements increasingly feasible.

\section{Particle Image Velocimetry}

PIV is an optical imaging technique for obtaining whole field velocities, therefore thousands of velocity vectors, instantaneously. The technique measures the motion of a fluid by observing the location of small tracers within the fluid at two distinct moments in time. The fundamental definition of velocity is the foundation for the technique.

$$
V=\frac{\Delta d}{\Delta t}
$$

The distance traversed by the tracer, $\Delta d$, is the quantity measured, while the time between observations, $\Delta t$, is specified. The method is applicable in gas and liquid flows. Flow tracers, often referred to as seed particles, are typically solid particles suspended in a gas or liquid or liquid droplets suspended in a gas. They may also include bubbles immersed in the fluid or solid particles with hollow cores.

Compared to typical point measurement techniques such as pitot-probes or LDV, PIV offers an instantaneous mapping of a specified flow field, increased spatial resolution, instantaneous information on velocity derivatives such as vorticity and strain, quantitative information about the spatial structure of the flow, and time averaged statistics of the velocity field. 
The standard PIV system will give two components of the velocity vector for a specified field. The method has further developed to offer all three components of the velocity vector for a specified field. The advanced feature technique, known as Stereoscopic PIV, has been applied in this study. PIV is a planar imaging technique that relies heavily on image post processing to infer the final results. Adrian [11] presents a good overview of the physical aspects of the technique, while Keane and Adrian [12] discuss the theory behind the image post processing ${ }^{1}$. In the sections to follow, a general summary of standard PIV will be presented first, followed by a brief description of the additional components and procedures necessary for Stereoscopic PIV.

\subsection{Two Dimensional PIV}

A standard PIV system, as displayed in Figure 2, consists of a double-pulsed laser to deliver two consecutive laser pulses coincident in space and separated in time $(\Delta t)$, sheet forming optics to expand the collimated laser beams into a planar sheets, a double-framed CCD (Charge Coupled Device) camera synchronized with each laser pulse and oriented perpendicular to the laser sheet, a data acquisition and control unit and a signal processing unit (frame grabber, computer and software).

Tiny seed particles which follow the flow are injected into the flow. The light sheet produced by the laser illuminates the region of interest in the flow with very short pulses of light, optically freezing the motion of the seed particles. The position of the seed particles is detected by the CCD camera which records two images, one for each pulse, of the scattered light from the seed particles. The particles appear in the images as white spots on a dark background (black and white images). The camera and the laser are synchronized so that particle positions at laser pulse 1 appear on camera frame 1 and particle positions at laser pulse 2 appear on camera frame 2. Images of the particle positions are sent to the computer for storage and signal processing.

\footnotetext{
${ }^{1}$ Details concerning the image analysis are omitted from the current discussion as it is a routine function of any PIV system and beyond the scope of this discussion.
} 


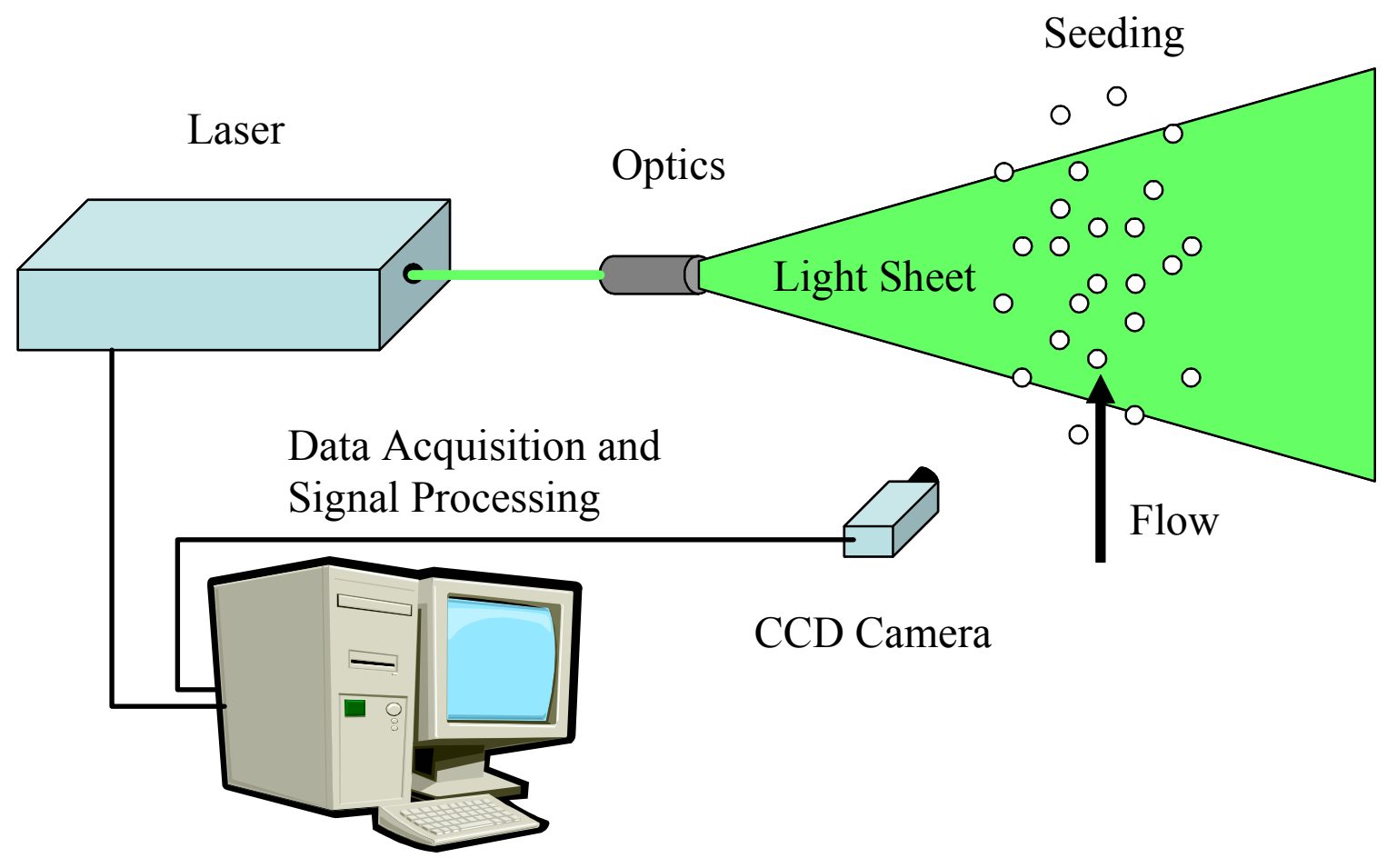

Figure 2 Basic PIV setup

Each image is divided up into square regions called interrogation areas. For each interrogation area, image 1 and image 2 are correlated to determine the average particle displacement over the interrogation area. This is repeated for all interrogation areas and a displacement vector map is created for the image space. Dividing the displacement vector map by the known $\Delta t$ between the laser pulses produces the velocity vector map. Figure 3 demonstrates this process.

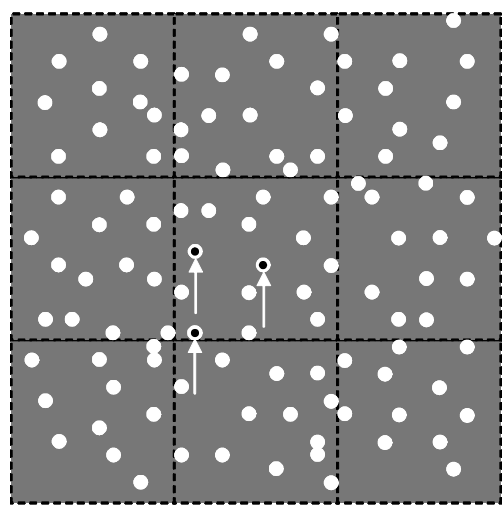

Frame 1

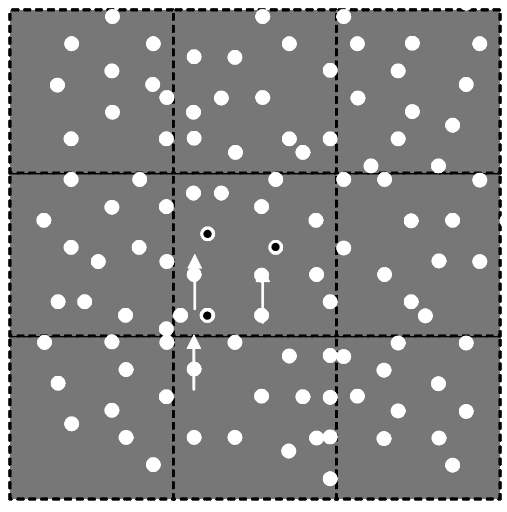

Frame 2

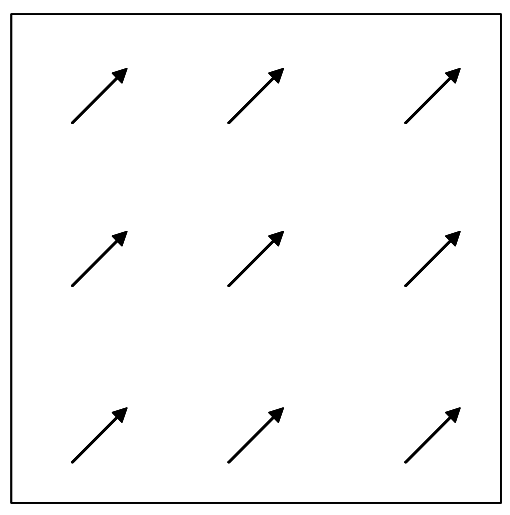

Velocity Vectors

Figure 3 Mean particle displacement is computed in each interrogation region (dashed squares) and converted to a velocity vector. The white arrows in frames 1 and 2 indicate the initial position of the selected particles (centered black dots). 


\subsection{Stereoscopic PIV}

In standard PIV the laser sheet is oriented so that the bulk motion of the flow runs parallel to the laser sheet and the optical axis of the CCD camera and collection optics is perpendicular to the laser sheet. In this configuration only the velocity vector in the plane of the laser sheet is measured; the out of plane component is unaccounted for. By introducing another camera to view the same area as the first camera but from a different angle, a setup is created which allows the out of plane velocity component to be determined. This configuration is called Stereoscopic PIV. Both cameras record different information about the same object in two-dimensions. The information can be combined to infer three-dimensional information.

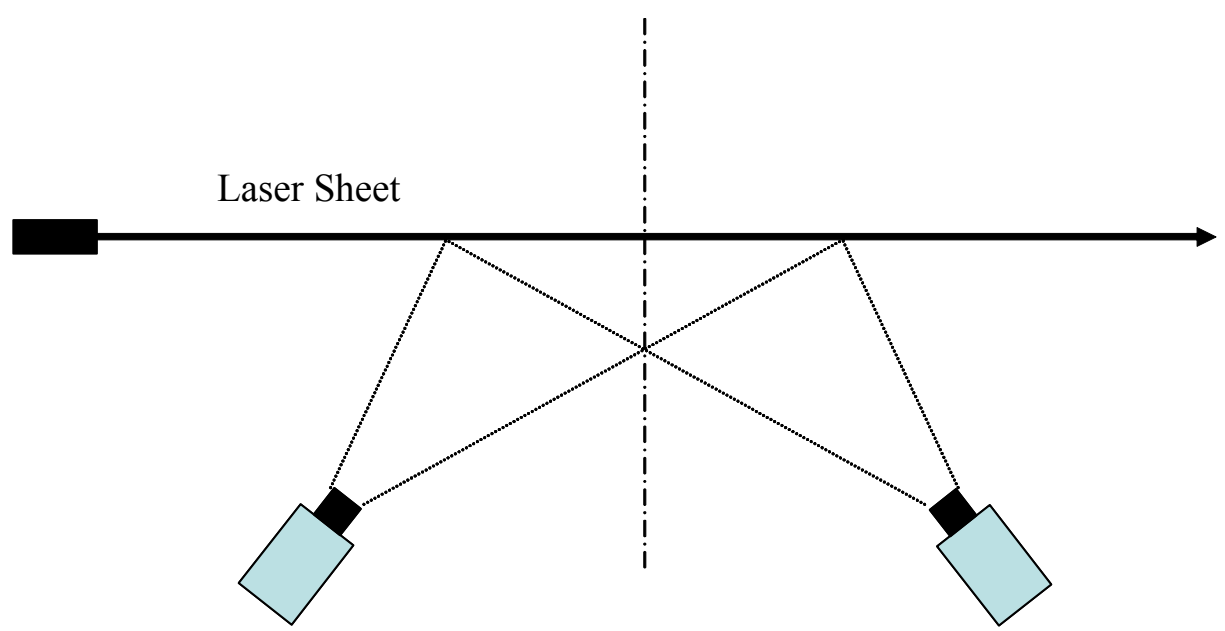

Camera 1

Camera 2

Figure 4 Typical Stereoscopic PIV configuration

A typical Stereoscopic PIV configuration, shown in Figure 4, places the cameras at 40 50 degrees off the axis perpendicular to the laser sheet and aligned to see the same field of view. ${ }^{2}$ A calibration target which consist of equally spaced black dots or crosses on a white background (or vise versa) is placed in the field of view and the cameras are focused on the target and aligned to get the maximum overlap between the two images. A sample target is shown in Figure 5.

\footnotetext{
${ }^{2}$ Other configurations are possible as described in the Stereoscopic PIV system manuals.
} 


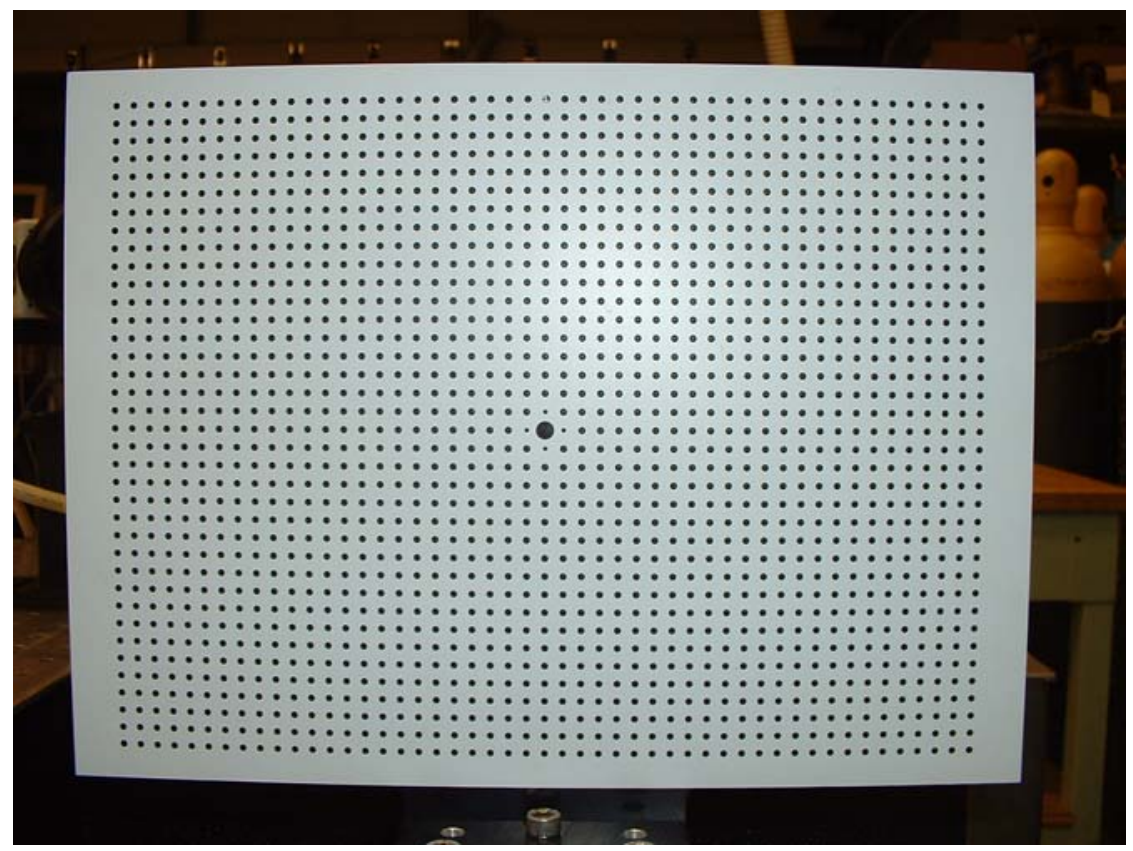

Figure 5 Target of black dots on white background used for Stereoscopic PIV calibration.

The calibration target is a well defined grid of markers used to create a set of transfer equations for the object in real space to the image space on the CCD chip. The target is aligned in the plane of the laser sheet, and as part of the calibration procedure it is traversed through the plane of the laser sheet at specified displacements in order to create the out of plane transfer equations. (Multilayered targets are also available to perform the same function without the need to traverse the target.) Additional analysis software, which is an add-on to the standard PIV analysis software, is required to process the stereo images and produce velocity vector fields with all three components of velocity. A stereo configuration adds complexity and effort, but it is an essential tool for measuring threedimensional flows.

\subsection{Large-Scale PIV}

PIV has been employed with great success in the laboratory for small to medium-scale flow fields that are well controlled. Flows inherent to full-scale fire experiments are on the scale of the size of the structure for enclosure fires. In addition the flow is buoyancy induced, and therefore is subject to the changing thermal conditions due to the fire. There is therefore limited knowledge about the flow structure of any given fire system before it has been studied. It is possible to exert control over the fire source (the source of buoyancy) and to some extent the physical boundary conditions. The physical dimensions of full-scale fire experiments push the limits for illuminating the test area, image collection, and seed particles selection. Fortunately the challenges in illumination and image collection are being met due to market forces which are credited with the great improvements in off-the-shelf laser and digital imaging technology. 


\subsubsection{Illumination}

Expanding the laser beam into a sheet across a large region reduces the intensity of incident light available for scattering by the seed particles. Therefore high pulse energy laser beams are recommended for large measurement areas. Typically $(150-200)$ $\mathrm{mJ} /$ pulse of laser energy for each beam is sufficient when illuminating a $1 \mathrm{~m} \mathrm{x} 1 \mathrm{~m}$ area. As a general rule of thumb, as much laser pulse energy as possible should be acquired within the fiscal, space, and supply power constraints of the experiment. It is possible to try to optimize laser pulse energy, particle size, and CCD chip responsivity to increase the scattering signal. The best recommendation is to maximize laser power and CCD responsivity at the laser wavelength to allow room for selecting smaller particles which are better flow tracers.

The laser for the current study is a Spectra Physics PIV-200. Laser pulse energy was measured at approximately $150 \mathrm{~mJ} /$ pulse for each laser beam. It is shown in Figure 6 with a light arm designed to allow high pulse energy laser beams to be safely delivered to the desired location.

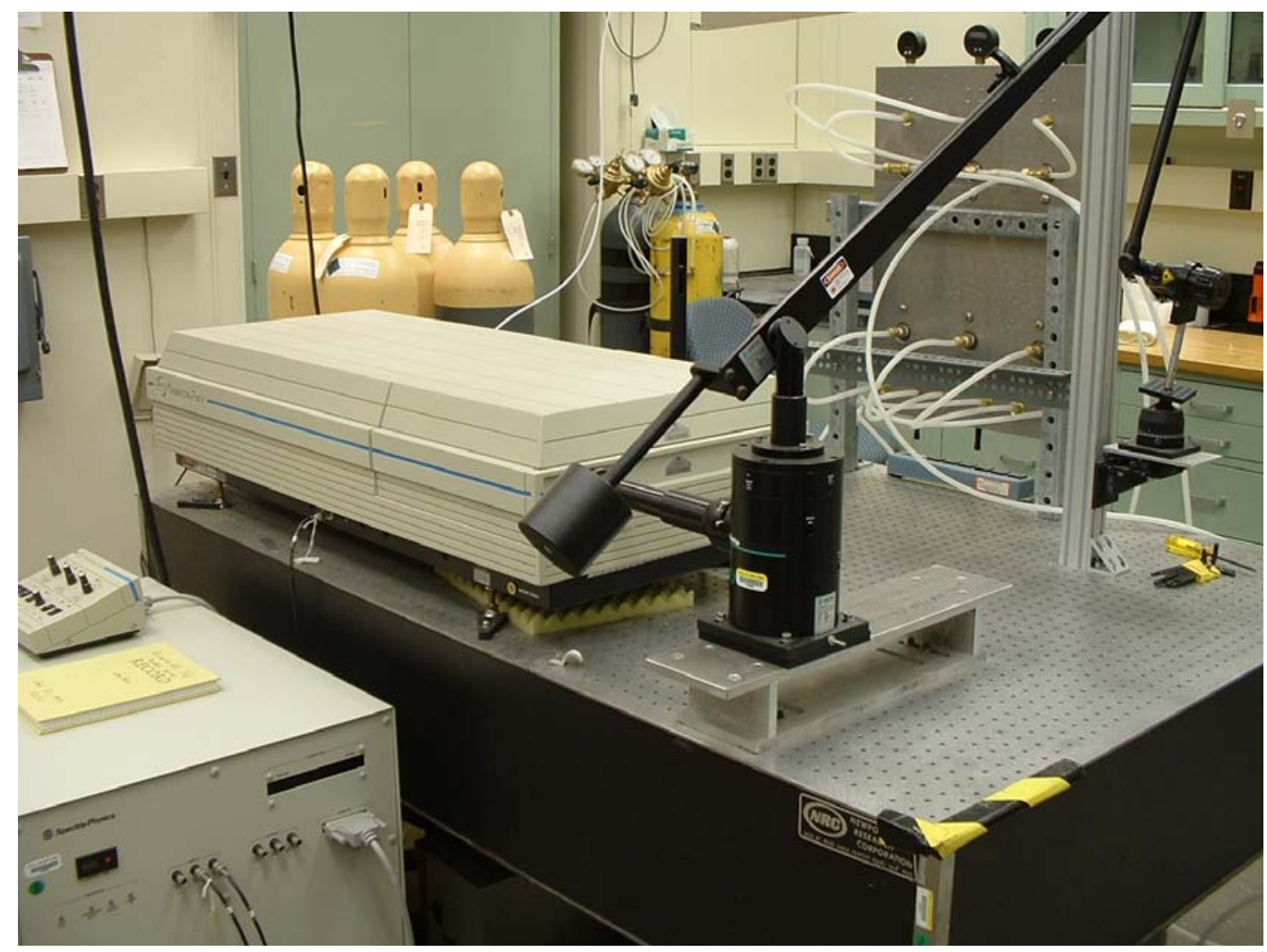

Figure 6 Double head Nd:YAG laser with light arm. 


\subsubsection{Seeding}

Seed particles are the simplest and perhaps the most important part of a PIV system. Seeding is essentially thousands of tiny probes suspended in the fluid that ride along with it. Without seeding, a measurement cannot be conducted. Therefore it is apparent why uniform seeding within a flow region is desirable. Uniformly seeding low speed flows that originate from the ambient surroundings is a challenging task.

\section{Seeding Density}

The cross correlation technique used to compute the particle displacements will produce some random noise in its computation. In order to keep the noise from dominating the results, several particle pairs have to exist within an interrogation region. Too low a number of particle pairs biases the results toward zero. Therefore the recommended minimum number of particle pairs per interrogation region is 5 for the cross correlation technique. Too high a seeding density may result in images where individual particles are not discernable. Ideal images will have particles appearing as discernable white spots on a dark background.

\section{Particle Selection}

It is a requirement that the seed particles follow the flow field. Ideally the seed particles should be neutrally buoyant for the flow conditions. If not neutrally buoyant, their terminal velocity and flow response time should be small compared to the velocity magnitudes expected and the time scales of the flow. At the same time the particle must provide sufficient light scattering intensity to allow detection by the CCD camera.

Financial limitations are often a constraining factor in experimental design. CCD cameras and lasers are the most expensive parts of a PIV system and both play a large part in seed particle selection. For large-scale PIV, image magnifications will be less than unity and the CCD cameras will be required to detect particles typically less than 1 $\mathrm{mm}$. Therefore determining if the CCD camera can detect candidate particles for a desired optical setup is a good place to start in selecting seed particles.

When the image of an individual particle is projected onto the CCD chip, it should cover at least 2 pixels. The CCD pixel pitch for the cameras employed in this study is $9 \mu \mathrm{m}$. Therefore the particle image should cover at least $18 \mu \mathrm{m}$. This allows the PIV analysis software to interpolate the position of the particle to within 0.1 pixels; a feature commonly referred to as sub-pixel interpolation. The predicted particle image diameter, $d_{\text {image }}$, is computed by the following relation which is a convolution of geometric optics and diffraction limited optics.

$$
d_{\text {image }}=\sqrt{d_{p}^{2} M^{2}+\left(2.44(1+M) f_{\#} \lambda\right)^{2}}
$$

The first term under the square root is the contribution from geometric optics where $d_{p}$ is the particle diameter and $M$ is the image magnification. The diffraction limited contribution to the image is the second term where $f_{\#}$ is the collection optics f-number and $\lambda$ is the wavelength of collected light. Figure 7 displays a few sets of curves 
generated from Equation 11, plotted with respect to image scale factor, SF, which is the inverse of the image magnification. The plot demonstrates that adjusting the collection optics f-number has a greater effect than choosing the particle size in meeting the 2 pixel coverage requirement. However, increasing the f-number reduces the lens aperture and therefore reduces the amount of light to be collected. So there are tradeoffs to be made.

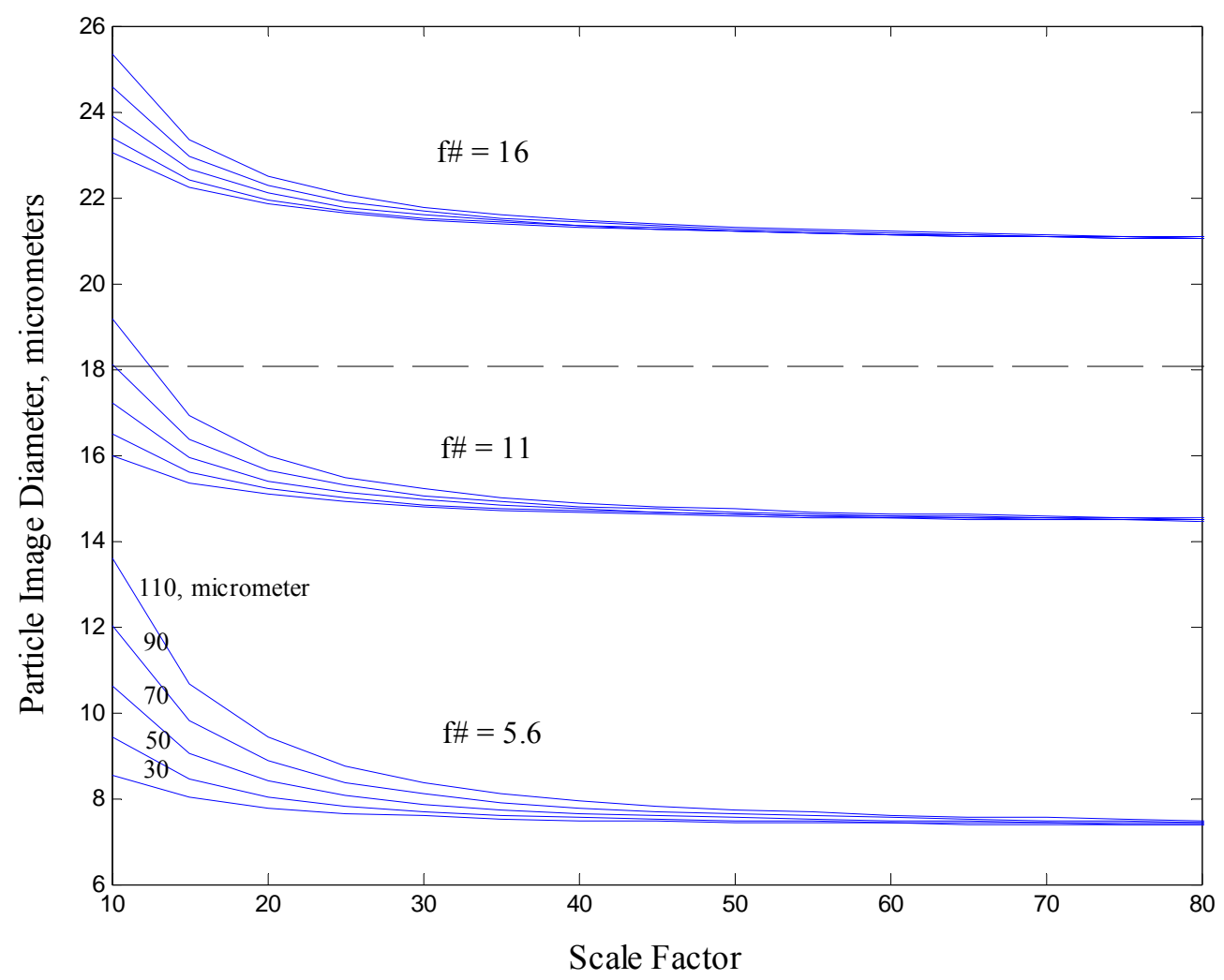

Figure 7 Particle image diameter plotted with respect to image scale factor, 1/M. Other important parameters are collection optics f-number and particle diameter.

For example in this experiment the seed particle diameters range from $(30-50) \mu \mathrm{m}$, the effective magnification is $0.014(\mathrm{SF}=70)$ and the wavelength of the scattered light is $0.532 \mu \mathrm{m}$. For an f-number of 5.6 the particle image diameter is $7.4 \mu \mathrm{m}$. Increasing the f-number to 16 reduces the collected scattering signal far too much. However, slightly defocusing the camera lens causes a blur which enlarges the particle image diameter to meet the 2 pixel coverage requirement. Note that selecting a CCD camera with a smaller pixel pitch may be a very expensive method of reaching the 2 pixel coverage requirement

In general, to reduce the cost associated with lasers and CCD cameras, larger particles are desired because they scatter more light and are more capable of being detected by the CCD camera. However, larger particles may not follow the flow as desired. If the seed particles are not neutrally buoyant they will either fall or rise in the flow. Depending on the magnitude of the flow velocity and the flow component of interest, particles that are 
not neutrally buoyant may be acceptable. Typical seed particles are solid or liquid aerosols and therefore when used in air are negatively buoyant, meaning that they are constantly falling in the flow.

When a particle falls in a surrounding fluid, the viscous drag exerts a force in the upward direction which balances the downward force of gravity on the particle. Once these forces are balanced a condition of constant velocity is created called the terminal velocity. Terminal velocity is estimated from the following equation for particle diameters greater than $1 \mu \mathrm{m}$ and particles having Reynolds number less than 1.0.

$$
V_{T}=\frac{\left(\rho_{p}-\rho_{g}\right) d_{p}^{2} g}{18 \mu}
$$

Estimates of terminal velocity were made for the $(30-50) \mu \mathrm{m}$ particles used in this study with a particle density, $\rho_{p}$, of $42 \mathrm{~kg} / \mathrm{m}^{3}$, a surrounding gas density, $\rho_{g}$, of 1.16 $\mathrm{kg} / \mathrm{m}^{3}$, and a gas viscosity, $\mu$, of $1.846 \times 10^{-5} \mathrm{~N} \mathrm{~s} / \mathrm{m}^{2}$, with $9.81 \mathrm{~m} / \mathrm{s}^{2}$ for the gravitational acceleration, $g$. Results range from $(0.0011-0.0030) \mathrm{m} / \mathrm{s}$ for the $(30-50) \mu \mathrm{m}$ particles respectively. At the low range of the horizontal velocity component measured in this study, the particle terminal velocity amounts to $1 \%$ or less of the horizontal component. A ratio of less than $1 \%$ is acceptable as a good rule of thumb for characterizing the seed particles as not being too negatively buoyant.

A particle must also sufficiently respond to abrupt changes in the flow to be considered a good flow tracer. The particle response time is another measure of a particles ability to follow the flow. Consider a particle suspended in a gas at rest and at time zero a pressure wave propagates through the gas, suddenly forcing it to move at some constant velocity. The particle must accelerate in order to catch up to the gas velocity. The time it takes for the particle velocity to reach $63 \%$ of the gas velocity is called the particle response time and is defined by the following equation.

$$
\tau=\frac{d_{p}^{2} \rho_{p}}{18 v \rho_{g}}
$$

For the case of a $50 \mu \mathrm{m}$ particle with a gas kinematic viscosity, $v$, of $1.59 \times 10^{-5} \mathrm{~m}^{2} / \mathrm{s}$, and the same particle and gas densities defined above, the particle response time is 0.3 ms. The time required for the particle velocity to equal $99 \%$ of the gas velocity is 4.6 times greater, $1.4 \mathrm{~ms}$. The characteristic time scales of the flows under consideration are on the order of seconds, therefore the particles are excellent candidates for following the flow.

Materials typically used for seeding range from liquid droplets to solid particles. Some of these materials are listed in Table 1. The particles selected for this study are dry expanded microspheres. The microspheres are filled with a gas, usually isopentane, and 
the shell is a copolymer of some monomers such as vinylidene chloride, acrylonitrile and methylmethacrylate. The particles are non-toxic and in bulk appear as a fluffy white dust. Proper respiratory protection should be worn when handling them. They are manufactured by Expancel (551 DE 40 d42) with a weight averaged diameter of (30 50) $\mu \mathrm{m}$, and a particle density of $42 \pm 4 \mathrm{~kg} / \mathrm{m}^{3}$.

Table 1 Typical seeding materials for PIV

\begin{tabular}{|c|c|c|c|}
\hline Material & $\begin{array}{c}\text { Size } \\
\text { Distribution, } \\
\mu \mathbf{m}\end{array}$ & $\begin{array}{l}\text { Recommended } \\
\text { Flow Medium }\end{array}$ & Comment \\
\hline $\mathrm{Al}_{2} \mathrm{O}_{3}$ & $<8$ & Air & $\begin{array}{l}\text { High temperature } \\
\text { applications }\end{array}$ \\
\hline Glycerin & $0.1-5$ & Air & $\begin{array}{l}\text { Generated by atomization, } \\
\text { water based, good light } \\
\text { scattering properties }\end{array}$ \\
\hline Silicone Oil & $1-3$ & Air & $\begin{array}{l}\text { High temperature } \\
\text { applications }\end{array}$ \\
\hline $\mathrm{SiO}_{2}$ & $1-5$ & Air & $\begin{array}{l}\text { High temperature } \\
\text { applications, spherical } \\
\text { particles }\end{array}$ \\
\hline Water & $1-2$ & Air & $\begin{array}{l}\text { Generated by atomization, } \\
\text { very inexpensive }\end{array}$ \\
\hline $\begin{array}{l}\text { Polymer } \\
\text { Microspheres }\end{array}$ & $30-50$ & Air & $\begin{array}{l}\text { Low density particles, good } \\
\text { light scattering properties }\end{array}$ \\
\hline $\begin{array}{l}\text { Hollow Glass } \\
\text { Microspheres }\end{array}$ & $2-20$ & Water & $\begin{array}{l}\text { Smooth spherical surface, } \\
\text { good light scattering } \\
\text { properties, high density }\end{array}$ \\
\hline Pine Pollen & $30-50$ & Water & $\begin{array}{l}\text { Oval shaped, inexpensive } \\
\text { and available in large } \\
\text { volumes }\end{array}$ \\
\hline
\end{tabular}

\subsubsection{Recommendations for Large-Scale PIV}

1) Use pulsed lasers with energy in excess of $150 \mathrm{~mJ} / \mathrm{pulse}$.

2) Select large pixel format CCD cameras with small pixel pitch (e.g. 2 mega pixels or greater, with $9 \mu \mathrm{m} \times 9 \mu \mathrm{m}$ pixels or less).

3) Optimize signal collection with wide angle collection optics; large $f_{\#}$ result in better image quality but reduced image intensity. Use aspheric optics to minimize the distortion for wide angle views.

4) Balance seed particle size with flow response; the larger the particle the greater the resulting image intensity, but the less responsive it is to the flow. 
5) Employ uniform seeding whenever possible; it is possible to achieve uniform seeding over time with a large sample of measurements.

6) Optimize seeding density to capture at least 5 particle pairs per interrogation region.

7) For large-scale fire measurements:

A remotely controlled seeding system is recommended as manual seeding will be unsafe.

Remote or triggered automatic control is also recommended since seeding is only required at the moments when measurements are conducted.

Mixing the seed particles into small air jets is an effective means of issuing seeding into the almost quiescent flow that enters the enclosure. (Small vacuum pumps that are driven by high pressure air are good candidates for mixing the solid particles and air.)

\section{Reduced-Scale Convective Analog Experiment}

The NIST Fire Research Division has established the goal of developing a methodology for PIV measurements in full-scale fire tests. This study serves as an important step in meeting this goal. PIV measurements of large-scale flows push the performance envelope of the typical off-the-shelf PIV system. Therefore, before going to full-scale fire experiments, attempting similar experiments at an intermediate scale under less harsh conditions was determined to be a necessary step. Steckler and his colleagues performed an extensive set of velocity measurements in the doorway of an enclosure with a constant-flow gas fire source which simulated the early stages of fire growth. $[9,13]$ Applying Froude number scaling, one experimental condition performed by Steckler et al. was simulated in this study with a helium plume as the source of buoyancy. The helium plume experiment was scaled to be 0.55 of the full-scale fire experiment. This reduced-scale convective analog only offered information on the flow and flow induced mixing of a similar real system. The performance of such a model is expected to be very good when heat transfer, chemical reactions, or boundary conditions are not important in the real experiment. Because the goal of this study is to demonstrate a flow measurement technique, a purely convective and isothermal analog is sufficient.

\subsection{Fire Experiment Scaling}

Fire scaling laws are typically derived from the non-dimensional Navier Stokes equations. The equations include non-dimensional coefficients such as Reynolds and Froude number which are ratios of relevant forces specific to the problem. If the nondimensional coefficients are kept invariant between the full-scale and reduced-scale experiments, both experiments are described by the same set of equations and hence their non-dimensional solutions are the same. Therefore proper scaling of geometrically similar fire experiments should have similar normalized spatial distributions of flow, temperature and composition. 
The helium plume is a low density gas relative to the ambient air. It is analogous to a high temperature buoyant fire plume. Since plume flows are buoyant the nondimensional equations describing them are governed by the Froude number, Fr.

$$
F r=\frac{\rho_{\infty} u^{2}}{\Delta \rho g l}
$$

The Froude number is the ratio of the inertia and buoyant forces, where $\rho_{\infty}$ is the ambient gas density, $\Delta \rho$ is the density difference between the plume and ambient gas, $u$ is the plume gas velocity, $l$ is the characteristic length scale (source diameter), and $g$ is the gravitational acceleration constant, $9.81 \mathrm{~m} / \mathrm{s}^{2}$. Therefore the Froude number must be preserved between the model and the real experiment.

Quintiere [14] and Steckler et al. [15] present a scaling analysis starting with the nondimensional equations describing a fire plume and an isothermal buoyant plume. By preserving the Froude number between the model and real-scale experiments the source terms, heat release rate, $\dot{Q}$, of the fire plume and the mass flow rate, $\dot{m}$, of the isothermal buoyant plume are related.

The Reynolds number of a turbulent fire plume is on the order of $10^{5}$. Steckler et al. estimate that if the Reynolds number of the reduced scale plume is on the order of $10^{4}$ or greater, the terms in the non-dimensional equations proportional to $1 / \operatorname{Re}$ may be neglected. The Reynolds number defined as $u l / v$, where $u$ is the characteristic velocity of the Froude scaling and $l$ is the height of the reduced-scale enclosure, was estimated to be on the order of $10^{4}$. Therefore the Reynolds number was ignored in the scaling analysis.

The scaling analysis presented in this report is more of a phenomenological one that picks up from the formal scaling of the non-dimensional equations describing the two systems and demonstrates how to apply the scaling to design the reduced-scale experiment.

In order to preserve the Froude number, a relation for the characteristic velocity, $u$, in terms of the experiment length scale, $l$, should be defined. Equation 14, implies that $u \sim$ $l^{1 / 2}$ and the following analysis will make use of this requirement.

The rate of heat released from a fire is equivalent to the advection of enthalpy away from the source

$$
\dot{Q}=C_{p} \Delta T \dot{m}_{\text {plume }}
$$

where, $C_{p}$ is the specific heat of the gas and $\Delta T$ is the temperature difference between the hot plume gases and the ambient air , $T-T_{\infty}$. For a highly ventilated fire plume the gases can be approximated as air, therefore $C_{p}=C_{p, a i r}$. For a perfect gas, the gas density, $\rho$, is inversely proportional to the gas temperature, $T$ and the following relation holds. 


$$
\frac{\Delta \rho}{\rho}=\frac{\Delta T}{T_{\infty}}
$$

This relation is significant because it illustrates the analogy of the temperature difference in the fire plume to the density difference in the isothermal helium plume. Recall that mass flow rate is defined as $\rho A u$, where $A$ is the cross sectional area and is proportional to $l^{2}$. A relation for the characteristic velocity can be defined from Equation 15 by substituting for the definition of mass flow rate and employing the temperature difference relation from Equation 16.

$$
u=\frac{\dot{Q}}{C_{p} T_{\infty} \Delta \rho l^{2}}
$$

The Froude number relation can now be written in terms of the heat release rate and the experiment length scale.

$$
F r=\left(\frac{\rho_{\infty}}{\Delta \rho}\right)^{3} \frac{1}{g C_{p}^{2} T_{\infty}^{2} \rho_{\infty}^{2}} \frac{\dot{Q}^{2}}{l^{5}}
$$

The density ratio was held constant for this experiment but it is a variable that may be used to maintain a constant Froude number. The second term is a constant for ambient conditions. The final term, $\dot{Q} / l^{5 / 2}$, was the control variable for this experiment and Froude number was preserved by keeping this ratio constant between the real experiment and the model. The subscripts FS and RS in Equation 19 refer to Full-Scale and Reduced-Scale respectively.

$$
\frac{\dot{Q}_{F S}}{l_{F S}^{5 / 2}}=\frac{\dot{Q}_{R S}}{l_{R S}^{5 / 2}}
$$

The equivalence of heat release rate and enthalpy advection, Equation 15, holds true for the reduced-scale plume as well. Since the reduced-scale system employs a helium plume as its source of buoyancy, it is a purely isothermal and convective analog of the full-scale system. Therefore the model should scale according to the convective heat release of the full-scale system, which is typically $0.7 \dot{Q}_{F S}$. [16] For a given heat release rate from a full-scale fire, the required mass flow rate for an analogous helium plume is computed using the following relation.

$$
\dot{m}_{H e}=\frac{0.7 \dot{Q}_{F S}}{C_{p} T_{\infty}}\left(\frac{l_{R S}}{l_{F S}}\right)^{5 / 2} \frac{\rho}{\Delta \rho}
$$


Since the goal of this study is to measure the flow through the doorway, the doorway height is used as the characteristic length scale $(l=H)$ of the experiments. The ratio of the doorway heights is the geometric scale factor $\left(l_{R S} / l_{F S}=H_{R S} / H_{F S}\right)$. All geometric dimensions are scaled according to this factor and its influence on the dynamic scaling appears in Equation 20. A doorway height of 1 meter was chosen for the reduced-scale experiment which is 0.55 times the full-scale height. This dimension is on the order of the measurement area limits of PIV and therefore exposes the challenges of applying PIV with a dynamically similar experiment. The relevant parameters for both the full-scale and reduced-scale experiments are shown in Table 2 for the conditions listed.

Table 2 Full-scale to reduced-scale parameter values.

\begin{tabular}{lcc}
\hline \multicolumn{1}{c}{ Parameter } & Full-Scale & Reduced-Scale \\
\hline Doorway Height, $H(\mathrm{~m})$ & 1.8 & 1.0 \\
Doorway Width, $W(\mathrm{~m})$ & 1.0 & 0.6 \\
Room Height, $h(\mathrm{~m})$ & 2.1 & 1.2 \\
Room Width, $w(\mathrm{~m})$ & 2.8 & 1.5 \\
Room Length, $I(\mathrm{~m})$ & 2.8 & 1.5 \\
Burner Diameter, $d(\mathrm{~m})$ & 0.31 & 0.17 \\
Heat Release Rate, $\dot{Q}(\mathrm{~kW})$ & 32 & 7.0 \\
Target Helium Mass Flow Rate, $\dot{m}_{\mathrm{He}}$ & - & 0.0026 \\
$(\mathrm{~kg} / \mathrm{s})$ & & \\
\hline$T_{\infty}=300 \mathrm{~K}, P_{a m b}=103 \mathrm{kPa}, \rho_{\text {air }}=1.1614 \mathrm{~kg} / \mathrm{m}^{3}, \rho_{\mathrm{He}}=0.1625 \mathrm{~kg} / \mathrm{m}^{3}, C_{p}=1.007 \mathrm{~kJ} / \mathrm{kgK}$
\end{tabular}

\subsection{Experimental Setup}

Conducting the experiments for this investigation required many steps. In addition many preliminary experiments such as flow meter confirmations, experiments to determine the best particle seeding, experiments to select the best optical configurations for the cameras, and experiments to estimate the optimum timing between laser pulses had to be performed. Once an optimum set of steps and conditions had been defined, the required measurements were conducted at the selected measurement planes.

The first step was to set up the stereoscopic PIV system. This required aligning the cameras so that each camera was focused on the same region. The calibration target was placed in the field of view of each camera and they were aligned so that the full target was in focus. The cameras were oriented to view the forward scatter from the particles as shown in Figure 8. The center of the target was imaged in almost the same position on each camera. This ensured that the overlap of the two images was nearly maximized. Next the calibration was performed to map the 3 dimensional object space to image space. The calibration was performed by traversing the target in the out-of-plane direction ( $z$ direction) relative to the laser sheet and recording images of the target at 5 to $9 \mathrm{z}$ positions. The target was returned to the center of the traversed distance and the laser sheet was aligned to vertically coincide with the center of the target (this was for a twosided target with both cameras viewing forward scatter, in the case that both cameras were viewing the same side of the target then the center of the laser sheet would be 
aligned to coincide with the front plane of the target). The laser sheet was spread over the imaged region with sufficient energy for scattering measurements.

As long as the cameras and laser sheet were not bumped or moved the calibration was valid. Any movement of the optical system required a new calibration to be performed. The enclosure was capable of being traversed around the optical setup. It was traversed in the $z$ direction to measure velocity vectors at 11 positions across the doorway. Because the entire height of the doorway could not be imaged, the enclosure was also traversed in the $y$ direction to completely map the velocity vectors in the full vertical height of the doorway.

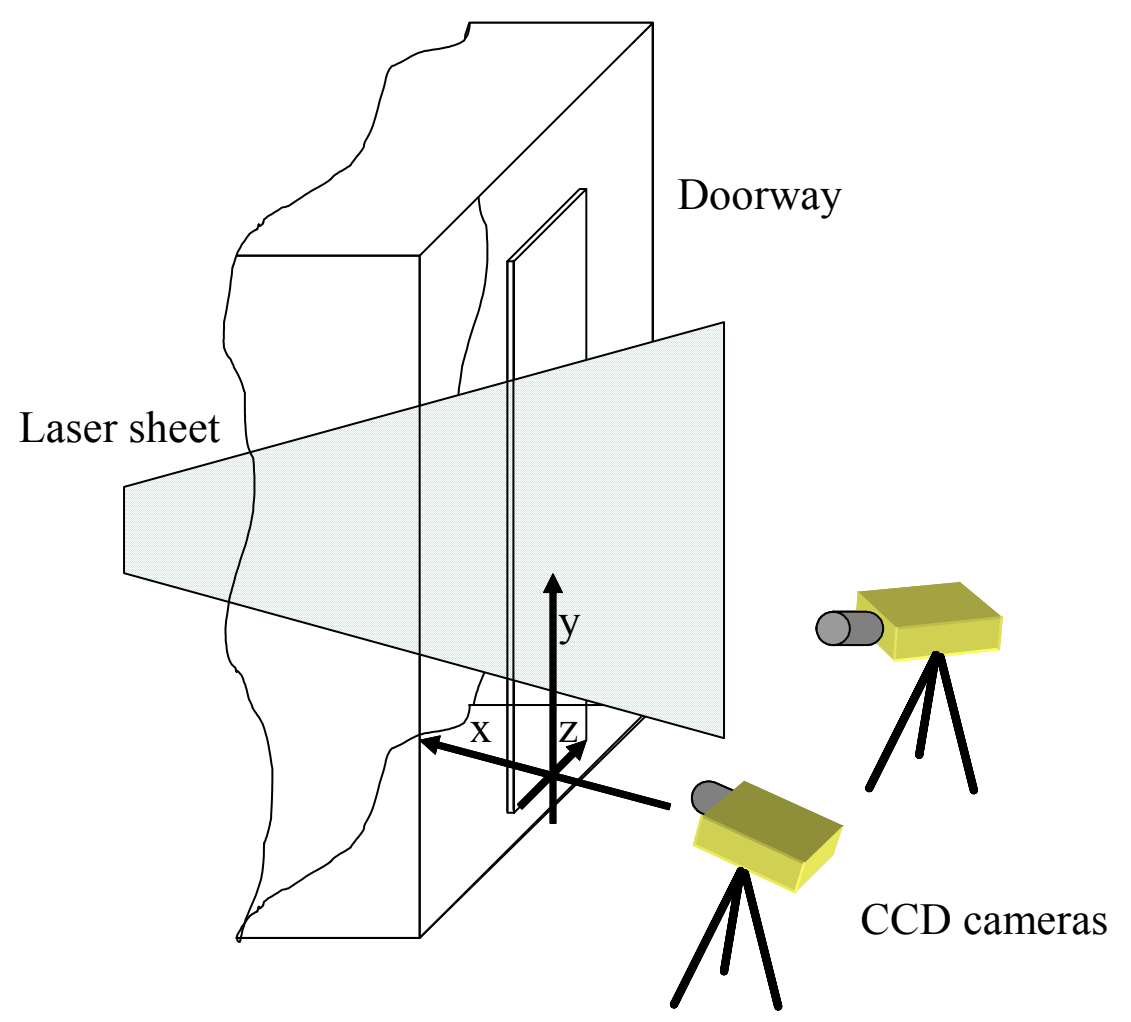

Figure 8 Optical configuration for the analog fire-enclosure experiments.

Starting at the $z=0 \mathrm{~cm}$ position, the helium flow was turned on to give a typical volume flow rate of 1133 SLM $(0.003 \mathrm{~kg} / \mathrm{s})$. Two high pressure helium bottles were run in parallel with separate regulators and flow meters. The helium flow was run for a maximum of 4 minutes and 1 minute was allowed to pass before any measurements were recorded (FDS results showed the flow at the doorway to be at steady state after about 40 seconds). ${ }^{3}$ At 40 seconds after the helium flow was started, seed particles were

\footnotetext{
${ }^{3}$ At this flow rate the experiment could ideally run continuously for approximately 10 minutes. However release of the high pressure gases causes the bottle temperature to decrease and therefore could influence the exit conditions at the helium plume. For this reason the helium flow was typically run for a maximum of 4 minutes which allowed two experiments to be conducted per pair of helium bottles.
} 
introduced to fill the region that supplied the inflow gas at the doorway. When the seeding was determined to be sufficient and the 1 minute duration had completed, the system was triggered to record images for velocity vectors. Three bursts of 20 measurements were recorded for a total of 60 velocity vector maps at each location. The three burst all occurred within the 4 minute helium flow duration. The image acquisition rate was set at $2 \mathrm{~Hz}$. Upon completion of the measurement the helium flow was shut down and the room was traversed to the next $\mathrm{z}$ location.

\subsubsection{Reduced-Scale Enclosure}

Visible light was required to pass through the walls of the enclosure in order to produce a laser sheet in the required measurement location and in order to observe the resulting scattering from the seed particles. Therefore the enclosure walls were constructed of clear Plexiglas of thickness $0.3 \mathrm{~cm}$, and the frame was constructed of aluminum angle (3.7 $\mathrm{cm}$ width on one side, $0.5 \mathrm{~cm}$ thickness). All vertical walls were formed from a single piece of Plexiglas, therefore no seams were present. The ceiling and the floor were constructed from two separate pieces with a single seam. The aluminum angle frame was located at all corners; a flat aluminum bar supported the ceiling and floor seams. The Plexiglas was attached to the frame using screws and two sided adhesive stripping (3M Scotch Brand Tape, $1.3 \mathrm{~cm}$ wide). At initial construction, the adhesive stripping provided a good seal for the enclosure, but as time passed the seals began to fail at locations away from the screw fasteners. These locations were fortified with duct tape.

The outside dimensions of the enclosure were $1.53 \mathrm{~m} \times 1.16 \mathrm{~m} \times 1.53 \mathrm{~m}(w \times h \times l)$. The doorway was located in the horizontal center of one end of the enclosure and $4 \mathrm{~cm}$ above the floor of the enclosure. The doorway dimensions were $0.54 \mathrm{~m} \times 1.0 \mathrm{~m}(w \times h)$. A hole was cut in the center of the floor of the enclosure and the helium plume inlet protruded through the hole to stand above the floor by $8.0 \mathrm{~cm}$. The outside diameter of the helium plume inlet was $19.0 \mathrm{~cm}$. A schematic of the enclosure with dimensions is shown in Figure 9 and a photograph of the enclosure is displayed in Figure 10. 


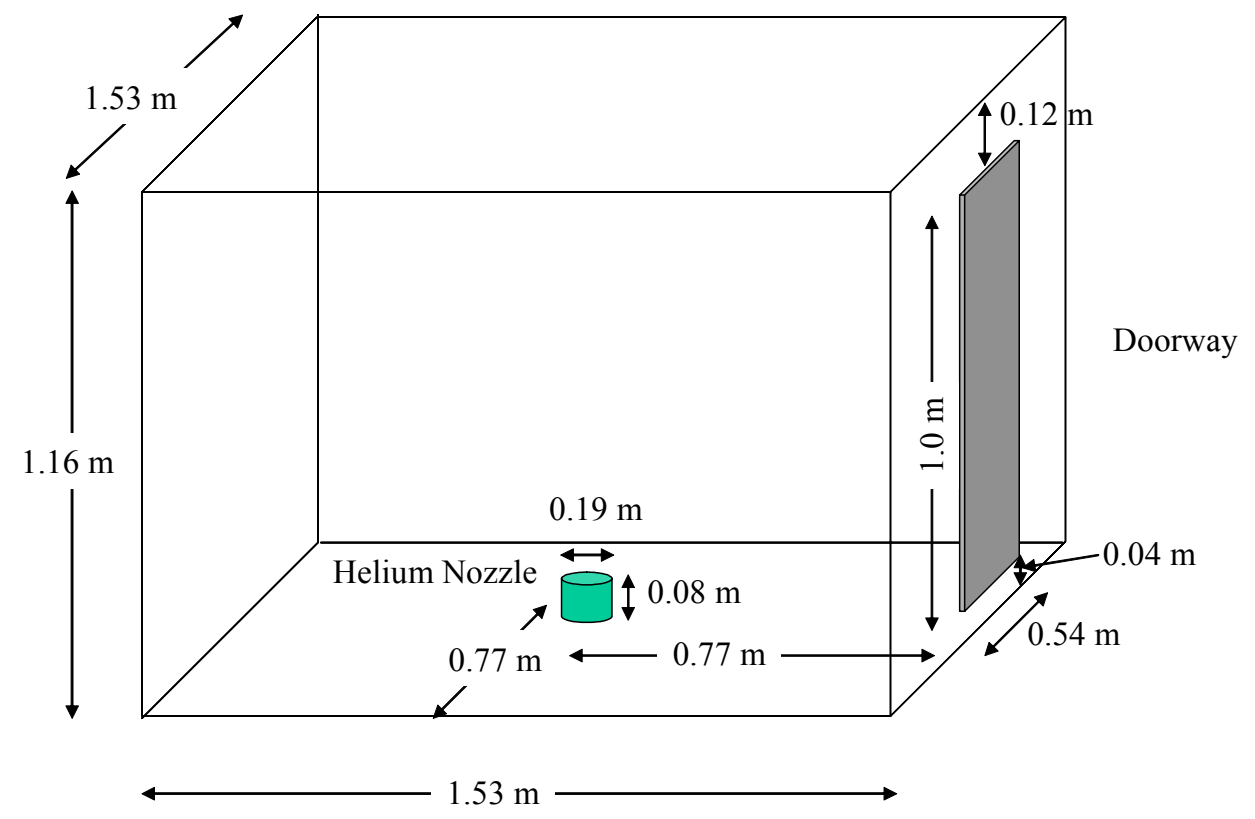

Figure 9 Schematic of reduced-scale enclosure with the helium plume inlet at the floor.

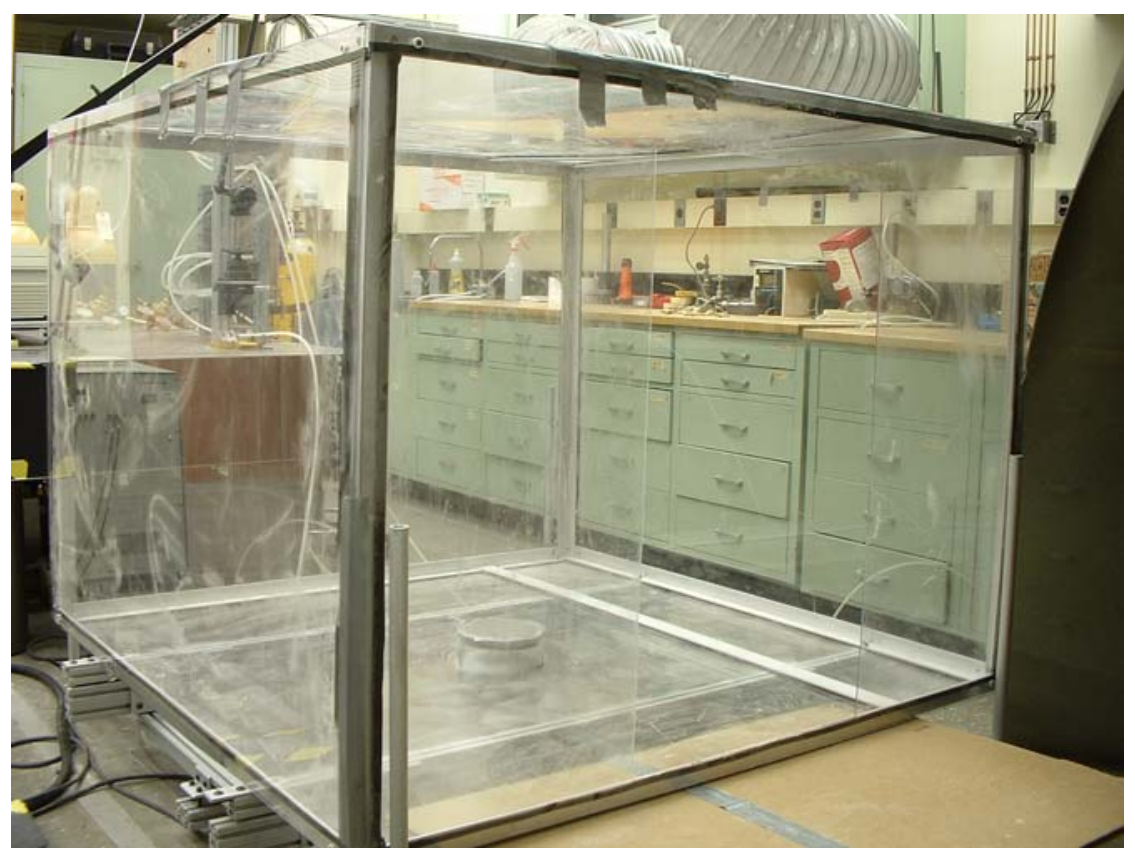

Figure 10 Photograph of reduced-scale enclosure.

Experiments with complicated optical setups are typically designed to keep the optical setup stationary and move the experiment relative to the optics. This was true for most of the experiments described in this report, however physical space limitations required the optics to be translated for a few experiments. A translating support mechanism was 
fabricated from extruded aluminum bar ( 80 / 20 Inc.) for moving the enclosure, Figure 11. The enclosure was placed on top of the support and securely attached. The support mechanism was moved horizontally by a hand crank attached to a threaded rod, which pushed and pulled the sliding supports. Vertical translation was accomplished by placing heavy duty laboratory jacks on both sides of the support mechanism to lower and raise the assembly. The sliding supports were locked in place once the desired vertical height was achieved. Because the enclosure was raised above the laboratory floor, a temporary floor was placed in front of the doorway to simulate the enclosure sitting on the floor.
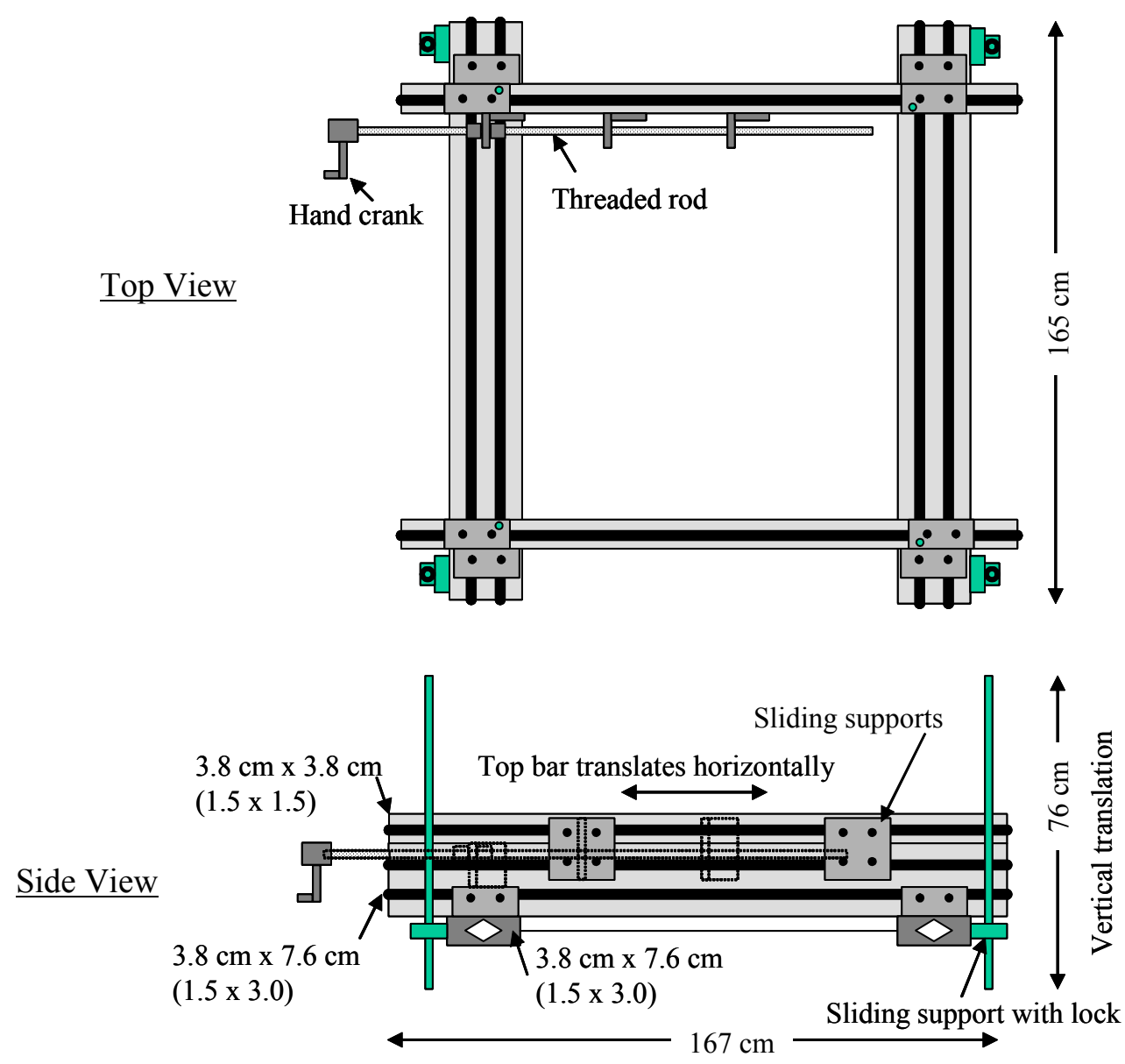

Figure 11 Schematic of translating support mechanism for the enclosure.

\subsubsection{Helium Flow System}

A flow system was fabricated to deliver helium from standard high pressure bottles (5.66 $\mathrm{m}^{3}$, at $13,790 \mathrm{kPa}$ ) to a circular exit near the floor of the enclosure. The flow was controlled by regulating the supply pressure from the bottles and next setting the volume flow rate by adjusting the variable area flow meters (Dwyer Rate Master, RMC-122-SSV, 566 SLM maximum flow rate (20 SCFM)). Pressure gauges (Ashcroft, $(0-207) \mathrm{kPa}((0$ - 30) psig)) were installed near the exit of the flow meters to provide pressure readings 
for correcting the flow meter readings. The tubing employed was $3 / 8$ " plastic tubing. A schematic of the flow system is shown in Figure 12.

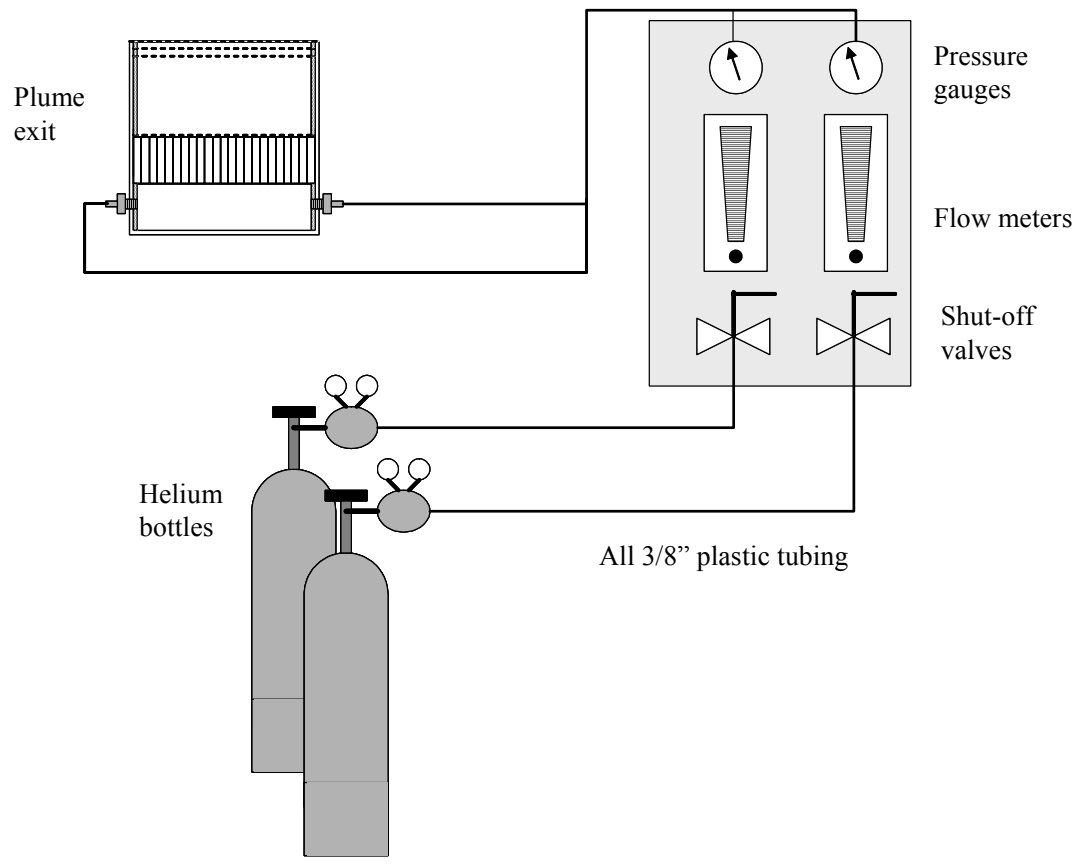

Figure 12 Helium plume flow system.

The helium plume exit assembly is shown in Figure 13. A Plexiglas tube $(19 \mathrm{~cm}$ o.d., 0.6 $\mathrm{cm}$ wall thickness) served as the outer shell of the plume assembly. A section of aluminum honeycomb $(0.35 \mathrm{~cm}$ cells $)$ located above the entry plenum provided flow straightening and 4 steel screens (200 mesh) served to provide flow uniformity. Smaller inner Plexiglas tubes $(16.7 \mathrm{~cm}$ i.d., $0.6 \mathrm{~cm}$ wall thickness) served as spacers to support the honeycomb and screens. Helium entered the flow plenum from two entry ports (total of 4 entry ports, the remaining two ports were closed). High frequency acoustic energy was produced by the helium issuing into the plume assembly. A layer of cotton balls was placed in the empty space above the honeycomb to absorb some of this acoustic energy and also to contribute to the flow uniformity. The helium plume exit assembly stands 25 $\mathrm{cm}$ high. It protruded by $8 \mathrm{~cm}$ through a hole cut in the enclosure floor. An adjustable platform below the enclosure and attached to the enclosure supported the plume exit assembly. A steel screen covered the exit of the plume assembly. The inner diameter at the exit was $16.7 \mathrm{~cm}$ due to the spacers for the screens. 


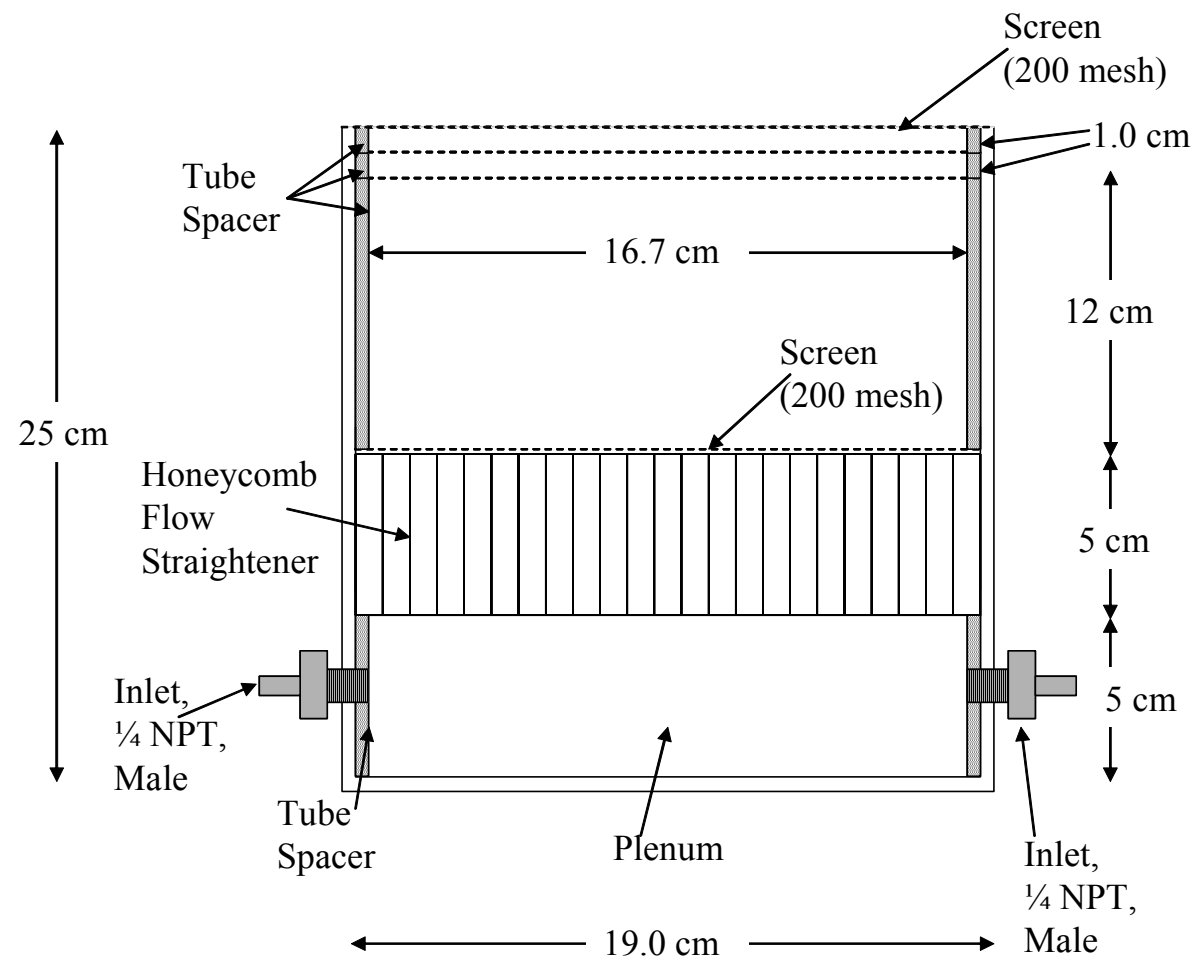

Figure 13 Helium plume exit assembly.

The variable area flow meters were calibrated for air by the manufacturer. Correction factors were applied to the scale reading, $\dot{V}_{\text {scale }}$, for the pressure within the flow meter, $P_{\text {flowmeter }}$, and for specific gravity, S.G., of the gas of interest. The actual volume flow rate of helium was determined using the following equation.

$$
\dot{V}_{H e}=\dot{V}_{\text {scale }} \sqrt{\frac{P_{\text {flowmeter }}}{P_{\text {amb }}} \frac{1}{S . G .}}
$$

The accuracy of the variable area flow meters was quoted as $\pm 2 \%$ of the full-scale reading. A dry test meter (Singer American Meter Division, DTM-200) was used to confirm the output from the flow meters. Figure 14 demonstrates the very good agreement between the measured output and the flow rate settings, therefore ensuring confidence in the operation of the flow meters. The flow meters regulate the flow for standard conditions, which are defined as $101325 \mathrm{~Pa}$ and $294 \mathrm{~K}$ by the manufacturer. The mass flow rate of helium was determined by computing the product of volume flow rate and helium gas density, $\rho_{\text {He,STP }}$, at this condition. 


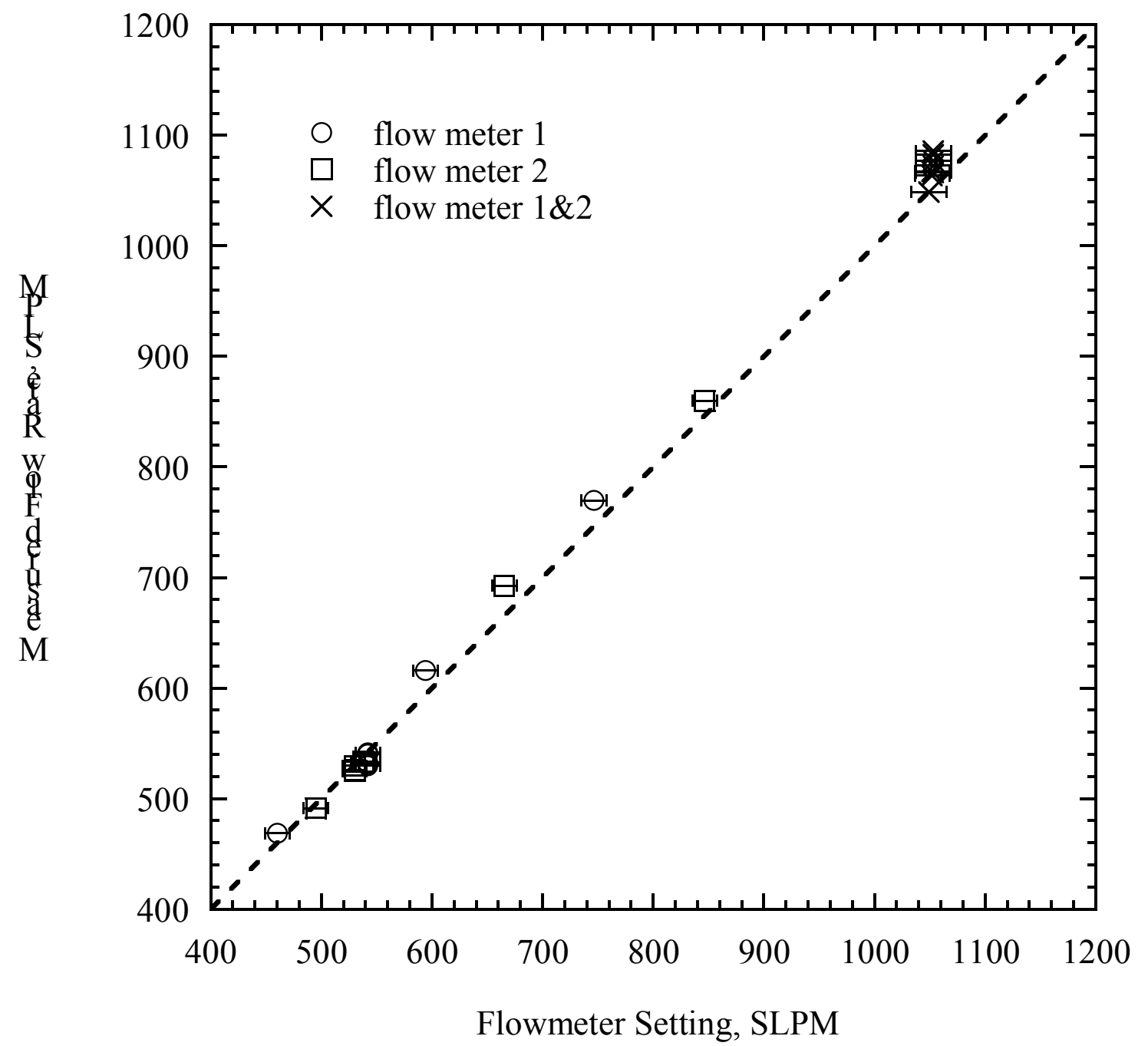

Figure 14 Helium flow confirmation.

\subsubsection{Stereoscopic PIV System}

A general description of a PIV system was presented in Section 3. A detailed description of the PIV system employed in this study shall follow. The Stereoscopic PIV system described here was supplied by Dantec Dynamics.

The laser was a pulsed Nd:YAG with two lasing heads (Spectra Physics PIV-200). The two lasing heads delivered two $532 \mathrm{~nm}$ beams that were coincident in space but separated in time. Each beam had an average pulse energy of $150 \mathrm{~mJ} /$ pulse at $532 \mathrm{~nm}$ (enough energy to rate the laser as a Class IV radiation hazard, i.e. eye protection was required at all times). The two lasing heads made possible the combining of two lasers in one package. Each laser beam pulsed at a rate of $15 \mathrm{~Hz}$ and the time between the two laser beam pulses was controllable from $5 \mu \mathrm{s}$ to $66 \mathrm{~ms}$. A light arm for high power lasers 
delivered the laser pulses from the laser exit port to the rear of the enclosure. Sheet forming optics were attached at the end of the light arm to spread the laser beams into a diverging set of light sheets which passed through the Plexiglas wall of the enclosure and illuminated the particles in the measurement region of the doorway. The laser sheets, like the laser beams, were coincident in space but separated in time.

The seed particles were dry expanded microspheres (Expancel 551 DE 40 d42). The microspheres were hollow plastic shells which enclosed a gas. They had a weight averaged diameter of $(30-50) \mu \mathrm{m}$ and a density of $42 \mathrm{~kg} / \mathrm{m}^{3}$. The seed particles were manually introduced into the flow using a shaker. The shaker was essentially a paint can with a fine mesh metal screen covering the opening. The experimenter stood about $1 \mathrm{~m}$ outside of the enclosure and was removed from the measurement location. The particles were introduced in a broad region so as to cover the incoming air with particles. The particles that entered the enclosure with the incoming air were entrained by the helium plume and transported to the upper layer to provide the outflow with seed particles. Therefore seeding from a single location provided ample seed particles for both the inflow and outflow.

Two cross-correlation CCD cameras (Kodak Megaplus ES1.0, Type 16) were employed in this PIV system. The term cross-correlation is used because the cameras were capable of recording two images, one for each laser pulse, and therefore offering a velocity measurement free of directional ambiguity. The minimum time between the two image frames was $1 \mu \mathrm{s}$. The CCD chip pixel dimensions were 1008 pixels x 1016 pixels. Each pixel had a pixel pitch of $9 \mu \mathrm{m} \times 9 \mu \mathrm{m}$, therefore resulting in CCD physical dimensions of $9.07 \mathrm{~mm} \times 9.14 \mathrm{~mm}$. The cameras were capable of frame rates up to 30 $\mathrm{Hz}$ in single frame mode and $15 \mathrm{~Hz}$ in double frame mode. The bit depth per pixel was 8 bits. This resulted in 256 grey levels. Attached to each camera was a $20 \mathrm{~mm}$ focal length Nikon AF Nikkor lens with $\mathrm{f}_{\#}$ of 5.6. The short focal length lens allowed for wide angle image views.

Stereoscopic PIV requires the cameras to be mounted at off-axis angles to the object plane as opposed to traditional PIV which has a single camera mounted on the axis of the object plane. In order to achieve the best plane of focus for this configuration, the camera image plane (CCD chip) must be rotated to simultaneously intersect with the lens plane and the object plane. This condition is known as the Scheimpflug condition and requires some distance between the lens and camera housing to allow for rotation. Special camera mounts were supplied which allowed the CCD chip plane to be rotated relative to the lens plane. The mounts also allowed the entire camera and lens to be rotated about the CCD chip vertical axis.

A calibration target was required to determine the object space to image space coordinate mapping. The calibration target was a grid of black dots on a white background. The dots were spaced $21.5 \mathrm{~mm}$ apart and had a diameter of $4.5 \mathrm{~mm}$. The center of the target was defined by a $6 \mathrm{~mm}$ dot surrounded by four $3 \mathrm{~mm}$ dots that defined the vertical and horizontal axis. The target was constructed of $90 \mathrm{~cm} \times 100 \mathrm{~cm}$ x $0.6 \mathrm{~cm}$ (height $\mathrm{x}$ width $\mathrm{x}$ thickness) Plexiglas sheet. Two identical dot patterns were adhered to both sides of the 
sheet to create a two sided target. The dot patterns covered an area of $77 \mathrm{~cm} \mathrm{x} 77 \mathrm{~cm}$. The sheet was held rigid by placing it in a square frame of $2.54 \mathrm{~cm} \times 2.54 \mathrm{~cm}$ extruded aluminum. This assembly was attached to a three axis traverse.

The data acquisition and control unit used to acquire the images from the CCD cameras and control timing between the experiment, lasers, and cameras was essentially a personal computer equipped with frame grabbers, an analog-to-digital converter, signal processing hardware, pulse synchronization hardware, and communications link to the user interface computer. The unit read images from the cameras to an input buffer. From this input buffer the images were temporarily stored in memory from which they could be sent directly to the computer hard disks for later processing or sent to the correlator unit which produced vector maps from the images in real time. The images were sent to the personal computer via an Ethernet communications board. The software (Flow Manager version 3.7) then stored the images and vector maps in a database, keeping track of all corresponding data acquisition and analysis parameters. The software was used for subsequent analysis of the vector maps, such as adaptive correlations and vector statistics.

The full detail of the Stereoscopic PIV system is presented in the "FlowMap Particle Image Velocimetry Instrumentation Installation and Users Guide" (Publication \#: 9040U3624, Date: 15 August 2000) and the "FlowMap 3D-PIV System Installation and User's Guide" (Publication no.: 9040U4112. Date: 16 August 2000) published by Dantec Dynamics. The reader is encouraged to review these documents for a complete description of the PIV system.

\subsubsection{Gas Sampling System}

Flow density was inferred from measurements of oxygen volume fraction in the flow stream. A gas sampling system, shown in Figure 15, was assembled for the oxygen volume fraction measurements. The system consisted of a stainless steel sampling probe (6.35 mm o.d., $4.57 \mathrm{~mm}$ i.d.) that was placed in the doorway plane at several different sampling locations. A volume displacement flow meter (Dry-Cal-Lite, DCLT 20k Rev1.08) was installed in the system to measure the sampling volume flow rate. The pressure at the exit of the flow meter relative to ambient pressure was monitored with a differential pressure gauge (Magnehellic, $\left.(0-981) \mathrm{Pa},\left((0-10) \mathrm{cm} \mathrm{H}_{2} \mathrm{O}\right)\right)$. A pump provided the pressure differential to pull a sample of gas from the flow and push it through the oxygen analyzer. The volume flow rate was controlled by a manual valve that was installed in the line. 


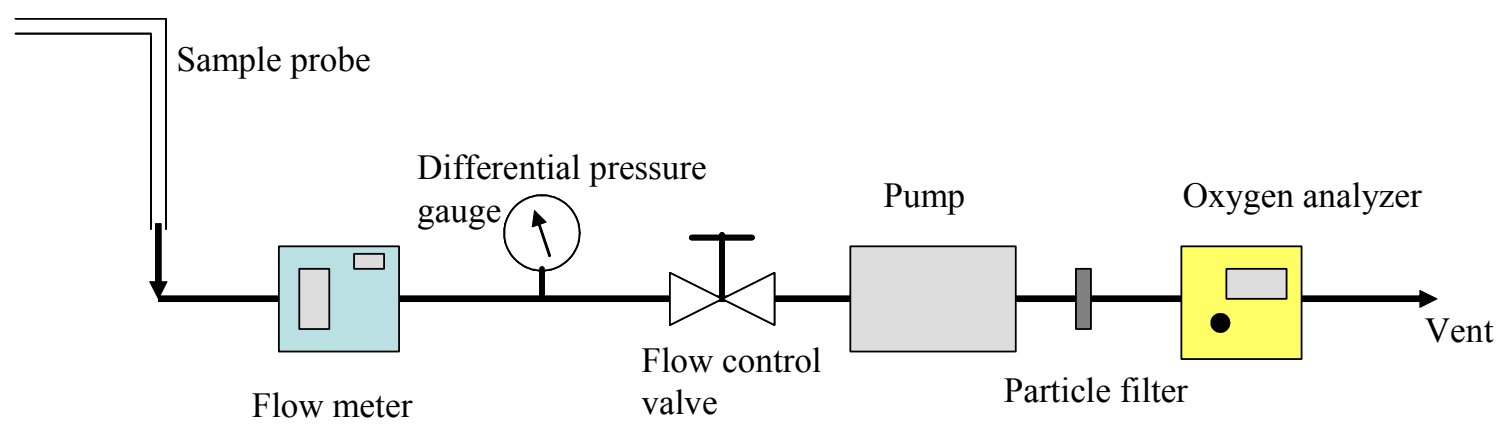

Figure 15 Gas sampling system to measure oxygen concentration.

The portable oxygen analyzer (Servomex 570A/712) was a paramagnetic device. Its voltage output ranged from 0.0 to $1.0 \mathrm{~V}$. Having a linear response to the oxygen volume fraction, an output of $1 \mathrm{~V}$ represented an oxygen volume fraction of 1.0. The output of the oxygen analyzer was monitored on a digital voltmeter and simultaneously recorded by a data acquisition system (Omega PDAQ56, OMB-DAQ-56) and stored on a personal computer. The sampling rate of the voltage signal by the data acquisition system was 1 $\mathrm{Hz}$.

\section{Uncertainty Analysis}

Measurement uncertainty is presented and follows the methodology described in the ISO guidelines and adopted by NIST. [17,18] Each component of uncertainty is presented as a standard uncertainty. So named Type A uncertainties are those that can be computed based on statistics, such as the standard deviation about the mean. Uncertainties that are determined from scientific judgment and other available information are designated as Type B uncertainties.

\subsection{Type A Uncertainty}

\section{Mean Velocity Measurement}

The velocity data presented are the mean values of at least 60 samples. The standard deviation about the mean is listed in Table 3. It is used to compute the standard uncertainty of the mean measurement, $u=\sigma / \sqrt{N}$. This value is the estimated standard uncertainty of the velocity measurement. The uncertainty estimates are divided among the two flow regions, inflow and outflow. These estimates are representative of 21 locations selected from the inflow region and 9 locations selected from the outflow region. 
Table 3 Mean velocity uncertainty estimates.

\begin{tabular}{ccccc}
\hline \hline Velocity & \multicolumn{2}{c}{ Inflow } & \multicolumn{2}{c}{ Outflow } \\
\cline { 2 - 5 } Component & $\sigma, \mathrm{m} / \mathrm{s}$ & $u\left(v_{i, \text { sample }}\right), \mathrm{m} / \mathrm{s}$ & $\sigma, \mathrm{m} / \mathrm{s}$ & $u\left(v_{i, \text { sample }}\right), \mathrm{m} / \mathrm{s}$ \\
\cline { 2 - 5 } & 0.057 & 0.007 & 0.152 & 0.020 \\
$\boldsymbol{v}_{\boldsymbol{x}}$ & 0.051 & 0.007 & 0.090 & 0.012 \\
$\boldsymbol{v}_{\boldsymbol{y}}$ & 0.060 & 0.008 & 0.151 & 0.020 \\
$\boldsymbol{v}_{\mathbf{z}}$ & &
\end{tabular}

\section{Oxygen Concentration}

The standard uncertainty is estimated using the sample standard deviation of repeat measurements. Measurements conducted near the neutral plane have a larger standard deviation due to the changing flow direction. Repeat measurements were conducted at this location in order to determine an upper bound on the standard deviation, Table 4. The standard deviation defines the standard uncertainty for a single measurement. This sample of measurements is used to estimate the uncertainty of all the measurements on centerline of the doorway. The estimate is conservative since the upper bound of the standard deviation of measurements is being applied.

Table 4 Sample oxygen concentration data near the neutral plane.

\begin{tabular}{|c|c|c|c|}
\hline $\mathbf{x}, \mathbf{m m}$ & $\mathrm{y}, \mathrm{mm}$ & $\mathrm{Z}, \mathbf{m m}$ & $X_{\mathrm{O}_{2}, \mathrm{SS}}$ \\
\hline-14 & 690 & 0 & 0.1969 \\
\hline-14 & 690 & 0 & 0.1919 \\
\hline-14 & 690 & 0 & 0.2016 \\
\hline-14 & 690 & 0 & 0.2010 \\
\hline-14 & 690 & 0 & 0.1942 \\
\hline-14 & 690 & 0 & 0.1959 \\
\hline \multirow[t]{3}{*}{-14} & 690 & 0 & 0.1999 \\
\hline & & Mean & 0.1973 \\
\hline & Standa & eviation & 0.0036 \\
\hline
\end{tabular}

\subsection{Type B Uncertainty}

\section{Temperature}

A stationary mercury thermometer with graduations of $1{ }^{\circ} \mathrm{C}$ was employed for the ambient temperature measurements, $T_{a m b}$. It is assumed that the temperature reading is described by a symmetric triangular probability distribution with upper bound $\left(T_{a m b}+1\right)$ ${ }^{\circ} \mathrm{C}$ and lower bound $\left(T_{a m b}-1\right){ }^{\circ} \mathrm{C}$. The standard uncertainty is then $u\left(T_{a m b}\right)=1 / \sqrt{6}=$ $0.48{ }^{\circ} \mathrm{C}$.

\section{Ambient Pressure}

The ambient pressure was read from a digital pressure indicator (Druck DPI 145). The expanded uncertainty of the instrument listed by the manufacturer is $\pm 0.014 \mathrm{kPa}$ over the range of $79.979 \mathrm{kPa}$ to $115.142 \mathrm{kPa}$. Assuming a coverage factor of 2, the standard uncertainty is estimated at $0.007 \mathrm{kPa}$. 


\section{Helium Volume Flow Rate}

The volume flow rate of helium, $\dot{V}_{H e}$, was controlled by variable area flow meters. The scale was divided into graduations of 0.5 SCFM (14.2 SLPM). The manufacturers reported accuracy is $\pm 2 \%$ of the full-scale reading, which results in an absolute accuracy of 0.4 SCFM (11.3 SLPM) for all readings. It is assumed that each volume flow rate reading is described by a symmetric rectangular probability distribution with upper bound $\left(\dot{V}_{\mathrm{He}}+0.4\right)$ SCFM and lower bound $\left(\dot{V}_{\mathrm{He}}-0.4\right)$ SCFM. The standard uncertainty is then $u\left(\dot{V}_{H e}\right)=0.4 / \sqrt{3}=0.24 \operatorname{SCFM}(6.8 \mathrm{SLPM})$.

\section{Dimensions}

The dimensions of the enclosure and measurement locations were measured using a flexible tape measure. The minimum graduation was $1 \mathrm{~mm}$. Considering the flexibility of the device and the skill of the operator, the standard uncertainty of all dimensions and measurement locations are estimated to be $1 \mathrm{~mm}$ unless otherwise stated.

$\underline{\text { PIV }}$

The uncertainty of a single PIV vector has contribution from a variety of sources such as the algorithm to compute the particle displacement, the particle response, particle seeding density, particle size distribution, and collection optics to name a few. Two contributions were estimated for this study, the uncertainty of the measured particle displacement and the particle response to the flow.

The quoted accuracy of the pixel displacement measurement was $1 / 10$ of a pixel. This resulted in a particle displacement uncertainty of $68 \mu \mathrm{m}$ for the CCD pixel pitch and image scale factor employed in this study, $9 \mu \mathrm{m}$ and 75.24, respectfully. The average time separation between PIV images was $7500 \mu \mathrm{s}$, which produces an estimated velocity uncertainty of $u($ algorithm $)=0.009 \mathrm{~m} / \mathrm{s}$.

The particles were estimated to respond almost instantaneously to the bulk flow relative to the characteristic time scales of the bulk flow. Therefore the uncertainty due to the temporal response of the particle is considered to be negligible. However the particles have a small terminal velocity in the vertical direction of $-0.003 \mathrm{~m} / \mathrm{s}$. This should add a bias to the measured vertical velocity component, but its contribution to the other components is unknown. Therefore the terminal velocity was considered as the uncertainty contribution for the particle response, $u($ PartResp $)=0.003 \mathrm{~m} / \mathrm{s}$.

\section{Oxygen Concentration Field Assumption}

Measurements of oxygen concentration were also conducted off centerline $(\mathrm{z} \neq 0 \mathrm{~mm})$ to estimate the dependence on horizontal location across the doorway, Table 5. The standard deviation from this sample of measurements serves as an estimate of the standard uncertainty in the assumption that the measured value on centerline at a specific elevation is representative of the value at any horizontal location across the doorway. 
Table 5 Sample oxygen concentration data at centerline $(\mathrm{z}=0 \mathrm{~mm})$ and off centerline.

\begin{tabular}{|c|c|c|c|}
\hline $\mathrm{x}, \mathrm{mm}$ & $\mathrm{y}, \mathrm{mm}$ & $\mathrm{z}, \mathrm{mm}$ & $X_{\mathrm{O}_{2}, \mathrm{SS}}$ \\
\hline-14 & 80 & 0 & 0.1735 \\
\hline-14 & 80 & 0 & 0.1730 \\
\hline-14 & 80 & 0 & 0.1759 \\
\hline-14 & 80 & 10 & 0.1746 \\
\hline-14 & 80 & 20 & 0.1708 \\
\hline-14 & 80 & 20 & 0.1728 \\
\hline \multirow[t]{3}{*}{-14} & 80 & 25 & 0.1779 \\
\hline & & Mean & 0.1741 \\
\hline & Standar & eviation & 0.0023 \\
\hline
\end{tabular}

Neutral Plane Height

The average position of the neutral plane was determined from a set of y locations meeting the criteria for the neutral plane. The standard deviation for this set of positions was $20 \mathrm{~mm}$ and serves as an estimate for the standard uncertainty of the neutral plane height.

\subsection{Uncertainty Propagation}

Suppose a general equation can be applied to describe a measurement process which has an output $y$ and the output $y$ is based on a number of input quantities, $x_{i}$.

$$
y=y\left(x_{1}, x_{2}, x_{3}, \ldots, x_{N}\right)
$$

In the case that all input quantities are uncorrelated, the combined relative expanded uncertainty is given by

$$
\frac{U_{e}(y)}{y}=k \frac{u_{c}(y)}{y}=k \sqrt{\sum_{i=1}^{N} s_{i}^{2}\left(\frac{u\left(x_{i}\right)}{x_{i}}\right)^{2}}
$$

In Eq. (23), $u\left(x_{i}\right)$ is the standard uncertainty for each input, $u_{c}(y)$ is the combined uncertainty, $k$ is the coverage factor, and $s_{i}$ is the associated dimensionless sensitivity coefficient given by

$$
s_{i}=\frac{\partial y}{\partial x_{i}} \frac{x_{i}}{y}
$$

\subsubsection{Helium Mass Flow}

Two variable area flow meters were used to set the helium volume flow rate. The mass flow rate of helium was determined by the following relation. 


$$
\dot{m}_{H e}=\left(\dot{V}_{H e, 1}+\dot{V}_{H e, 2}\right) \rho_{H e, S T P}
$$

Volume flow rate and helium density, $\rho_{H e, S T P}$, were both referenced to the standard conditions of $101325 \mathrm{~Pa}$ and $294 \mathrm{~K}$ as defined by the flow meter manufacturer. Applying the standard uncertainty estimates for each volume flow rate measurement to the general equation for uncertainty propagation, the relative combined uncertainty of the helium mass flow rate is $u\left(\dot{m}_{H e}\right) / \dot{m}_{H e}=0.01$. A coverage factor of 2.0 is applied, resulting in an expanded relative uncertainty of \pm 0.02 .

\subsubsection{Mean Velocity Field}

Since there is not one simple equation to describe the velocity measured with Stereoscopic PIV, each of the uncertainty components described previously were added in quadrature to estimate the uncertainty of the mean velocity field, $u\left(v_{i}\right)=\sqrt{u(\text { a } \lg \text { orithm })^{2}+u(\text { Part } \operatorname{Re} s p)^{2}+u\left(v_{i, \text { sample }}\right)^{2}}$.

Table 6 Mean velocity field uncertainty estimates.

\begin{tabular}{ccc}
\hline \hline Velocity & \multicolumn{2}{c}{$u\left(v_{i}\right), \mathrm{m} / \mathrm{s}$} \\
\cline { 2 - 3 } Component & Inflow & Outflow \\
\cline { 2 - 3 } $\boldsymbol{v}_{\boldsymbol{x}}$ & 0.012 & 0.022 \\
$\boldsymbol{v}_{\boldsymbol{y}}$ & 0.012 & 0.015 \\
$\boldsymbol{v}_{\boldsymbol{z}}$ & 0.012 & 0.022 \\
\hline
\end{tabular}

\subsubsection{Mean Gas Density Field}

The mean gas density field is inferred from the oxygen concentration measurements as defined by Equation 31. Uncertainty estimates for the mean oxygen concentration measurements are derived from data in Table 4 and Table 5. The relative uncertainty of the mean computed from Table 4 is 0.007 and represents the relative standard uncertainty for the mean vertical profile for the center of the doorway, $X_{\mathrm{O}_{2}}(y, z=0)$. The entire oxygen concentration field was approximated by the centerline profile ( $X_{\mathrm{O}_{2}}(y, z) \approx X_{\mathrm{O}_{2}}(y, z=0)$ ); a valid assumption as demonstrated by the relatively low standard deviation of the measurements in Table 5. The relative uncertainty of the mean was computed from this sample, 0.005 , to estimate the relative standard uncertainty of this assumption. Both relative standard uncertainties were added in quadrature and the relative combined uncertainty of the oxygen concentration field was estimated to be 0.009 . Applying the general equation for uncertainty propagation, the relative combined uncertainty of the density field is estimated to be 0.009 . A coverage factor of 2.0 is applied, resulting in an expanded relative uncertainty of \pm 0.018 . 


\subsubsection{Doorway Mass Flow Rate}

The mass flow rate of fluid into and out of the enclosure was computed using a numerical integration technique, the Trapezoid Rule (See Appendix). The technique is essentially the product of the length of the integration interval and the mean value of the integrand over the integration interval. A double integration estimated by the Trapezoid Rule is therefore the product of the area defined by the integration intervals and the mean value of the integrand over the defined area. For the purpose of estimating the uncertainty, the numerical integrals to compute mass flow rate were approximated by the following relations.

$$
\begin{gathered}
\dot{m}_{\text {in, exp }} \approx \rho_{\text {avg, }, \mathrm{n}} v_{\text {xavg,in }} W h_{N} \\
\dot{m}_{\text {out, exp }} \approx \rho_{\text {avg, out }} v_{\text {xavg,out }} W\left(H-h_{N}\right)
\end{gathered}
$$

The mean gas density and gas velocity were computed from the spatial field of measurements. The relative standard uncertainty of the gas density field was defined in Section 5.3.3. The standard uncertainty of the mean velocity of the region was computed by normalizing the standard uncertainty defined in Table 6 by the square root of the number of measurement locations within the region. Nominal values of the parameters for Equations 26 and 27 are listed in Table 7 along with estimates of their relative standard uncertainty. The general equation for propagation of uncertainty was applied to estimate the relative standard uncertainty of doorway mass flow rate. A coverage factor of 2 is applied to compute the relative expanded uncertainty.

\begin{tabular}{|c|c|c|c|c|}
\hline Parameter & $\begin{array}{l}\text { Nominal } \\
\text { Value } \\
\text { inflow }\end{array}$ & $\begin{array}{l}u\left(x_{i}\right) / x_{i} \\
\text { inflow }\end{array}$ & $\begin{array}{c}\text { Nominal } \\
\text { Value } \\
\text { outflow }\end{array}$ & $\begin{array}{l}u\left(x_{i}\right) / x_{i} \\
\text { outflow }\end{array}$ \\
\hline$\rho_{a v g}, \mathrm{~kg} / \mathrm{m}^{3}$ & 1.165 & 0.009 & 0.991 & 0.009 \\
\hline$v_{x a v g}, \mathrm{~m} / \mathrm{s}$ & 0.187 & $\begin{array}{c}0.002 \\
(N=693)\end{array}$ & -0.342 & $\begin{array}{c}0.004 \\
(N=330)\end{array}$ \\
\hline$W, \mathrm{~m}$ & 0.54 & 0.002 & 0.54 & 0.002 \\
\hline$h_{N}, \mathrm{~m}$ & 0.69 & 0.029 & - & \\
\hline$\left(H-h_{N}\right), \mathrm{m}$ & - & - & 0.31 & 0.065 \\
\hline$u_{c}\left(\dot{m}_{\exp }\right) / \dot{m}_{\exp }$ & & 0.030 & & 0.066 \\
\hline
\end{tabular}

Table 7 Doorway mass flow rate relative standard uncertainty estimates.

\section{Results of Reduced-Scale Experiments}

\subsection{Vector Processing}

\subsubsection{Instantaneous Measurements}

The steps involved in running the experiment were described in Section 4.2. Generating vector maps from the CCD image maps involved another set of steps that made use of the 
software provided by the PIV manufacturer. Figure 16 is an example of typical images of the seed particles recorded during an experiment. Both cameras recorded two images separated by a specified $\Delta t$. Approximately 60 acquisitions of such image pairs were recorded at each measurement location. The cameras viewed the same field but from different sides of the laser sheet. In the images below, the inflow (solid arrow) is more heavily seeded with particles since they are introduced outside of the enclosure. The particle laden air flowing into the enclosure mixed with the helium plume to provide seeding for the outflow. Note that even though the outflow seeding density was less than the inflow, it was still sufficient to produce good quality velocity vectors. The lower seeding density was due some dilution of incoming particle stream by the unseeded helium plume but it is mostly due to particles falling out of the flow before being entrained into the upper layer. Regions where the laser sheet passed through the walls and caused strong reflections appeared as bright saturated pixels within the images. Computation of true velocity vectors from these regions was not possible. Use of advanced computation techniques could, however, produce substituted velocity vectors.

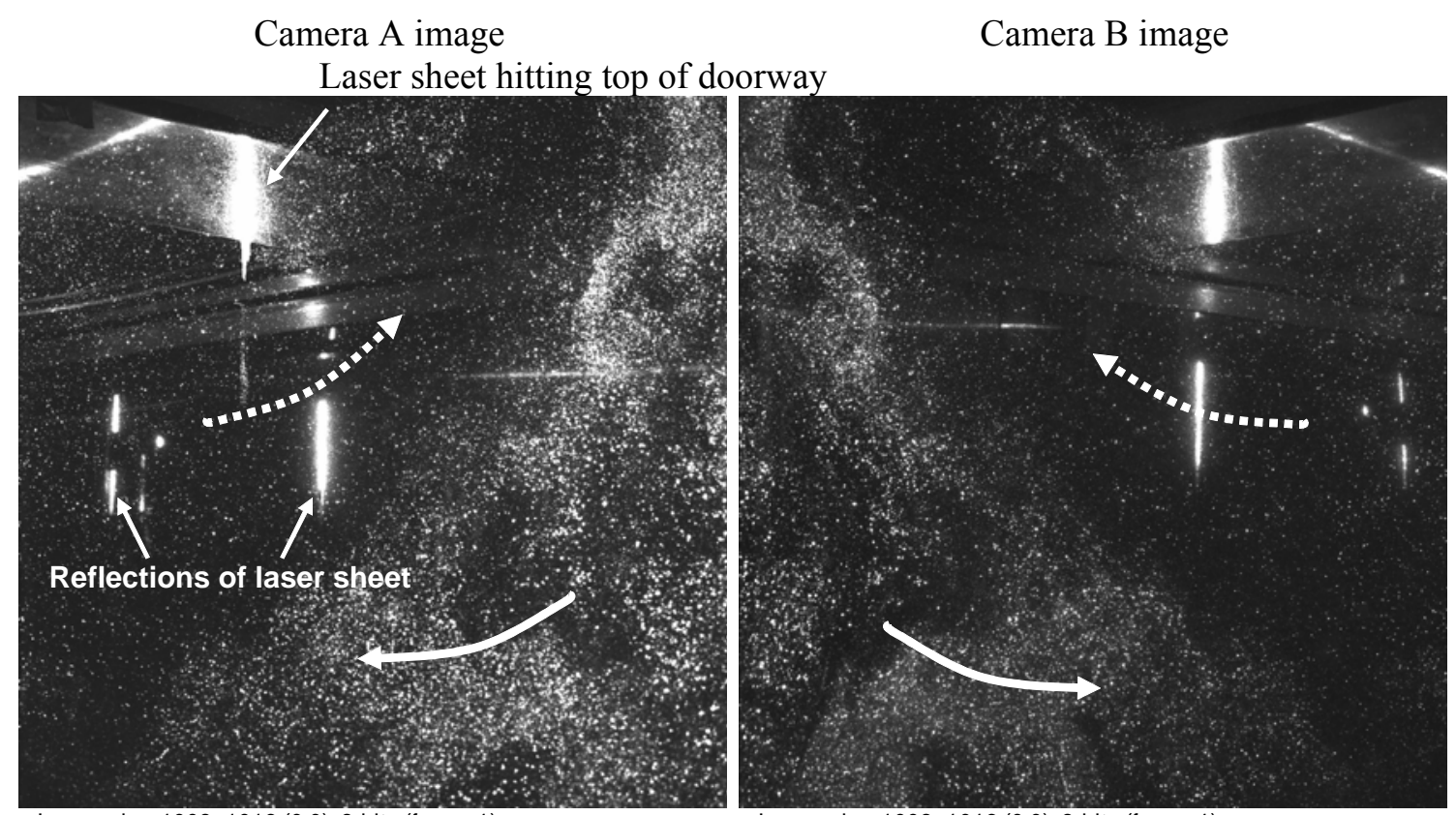

Figure 16 Particle images from cameras A and B. Solid arrow represents inflow dotted arrow represents outflow.

The PIV system was capable of displaying in real time the displacement vectors as the images were acquired. Figure 17 is an example of what the experimenter saw during a measurement. The displacement vectors were computed using the standard cross correlation of the image maps with a $50 \%$ overlap of the interrogation regions. The vector field was $64 \times 64$ vectors due to the $50 \%$ overlap; however only $32 \times 32$ vectors are shown in the figures below for the sake of clarity. Blue vectors met the validation criteria while the red vectors did not and were therefore rejected. Rejected vectors occurred in areas of the image map where the seeding density was too low or the pixels 
were saturated. The vector maps in Figure 17 were produced with a basic level of post processing in order to provide the experimenter some immediate feedback on the quality of the measurements.

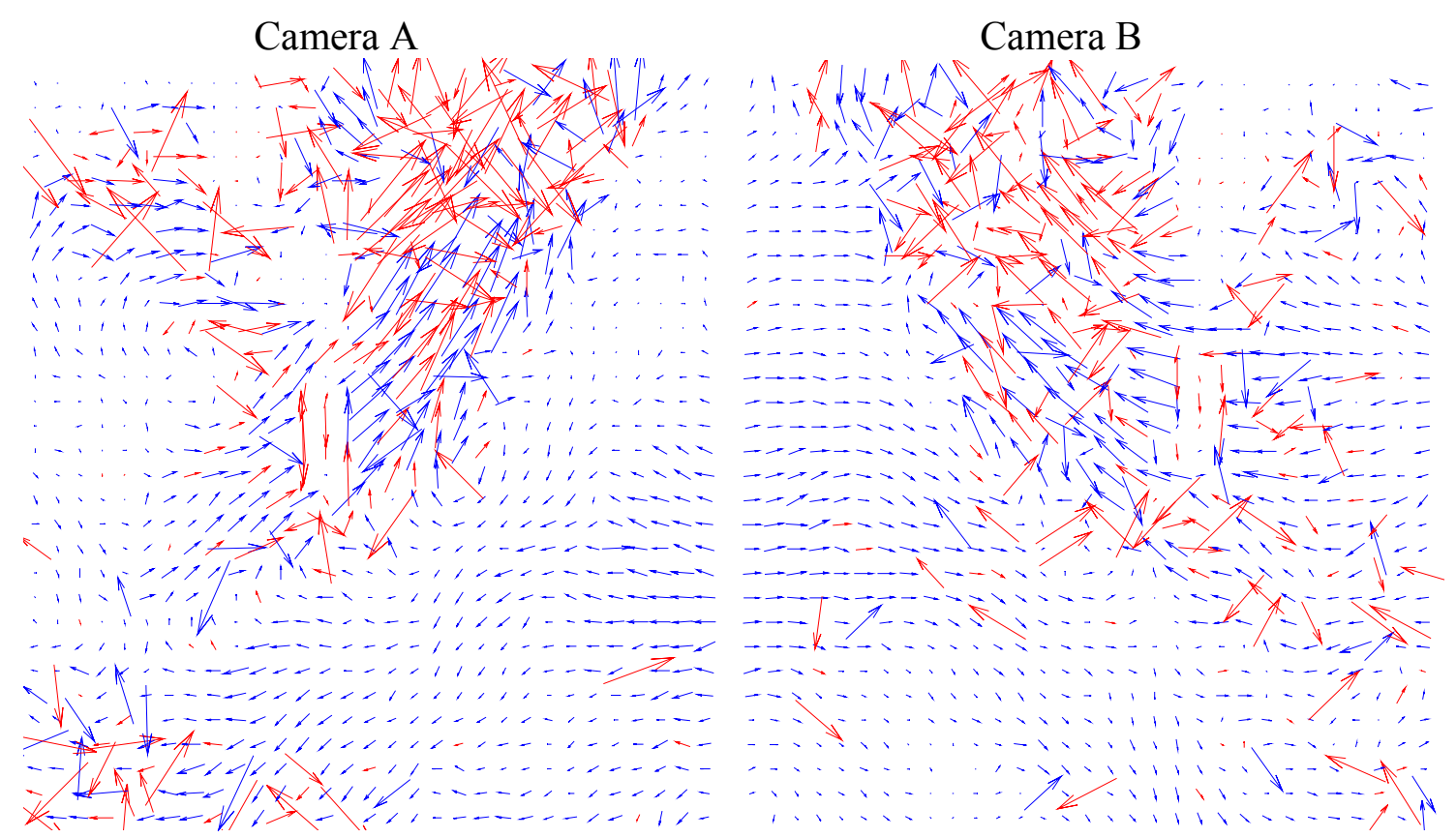

Figure 17 Displacement vectors computed from Figure 16 images A and B using the standard cross correlation technique.

An advanced method of post processing called adaptive cross correlation was performed on the image maps in order to achieve a higher quality of data. The adaptive cross correlation method began with an interrogation region three times the size of the final interrogation region. This helped to reduce the number of rejected vectors due to low seeding density and particles leaving the interrogation region due to long laser pulse separations. Multiple iterations of the cross correlation were applied and the average displacement vector computed. On the next iteration of cross correlations, the interrogation region was shifted by an amount determined from the displacement results of the previous cross correlation. Therefore, the shift in interrogation regions was not constant but adaptive to the magnitude and direction of displacements measured on a previous pass. This process of multiple passes and shifting the interrogation regions was repeated for a specified number of iterations, and in addition, the size of the interrogation regions was reduced and the steps were performed again. This continued until the final specified interrogation region was achieved. Figure 18 is an example of the displacement vectors computed with the adaptive cross correlation technique. Like with the standard cross correlation technique the blue vectors represent valid vectors. The green vectors represent vectors that have been computed by interpolation of the information from the valid vectors surrounding them. 


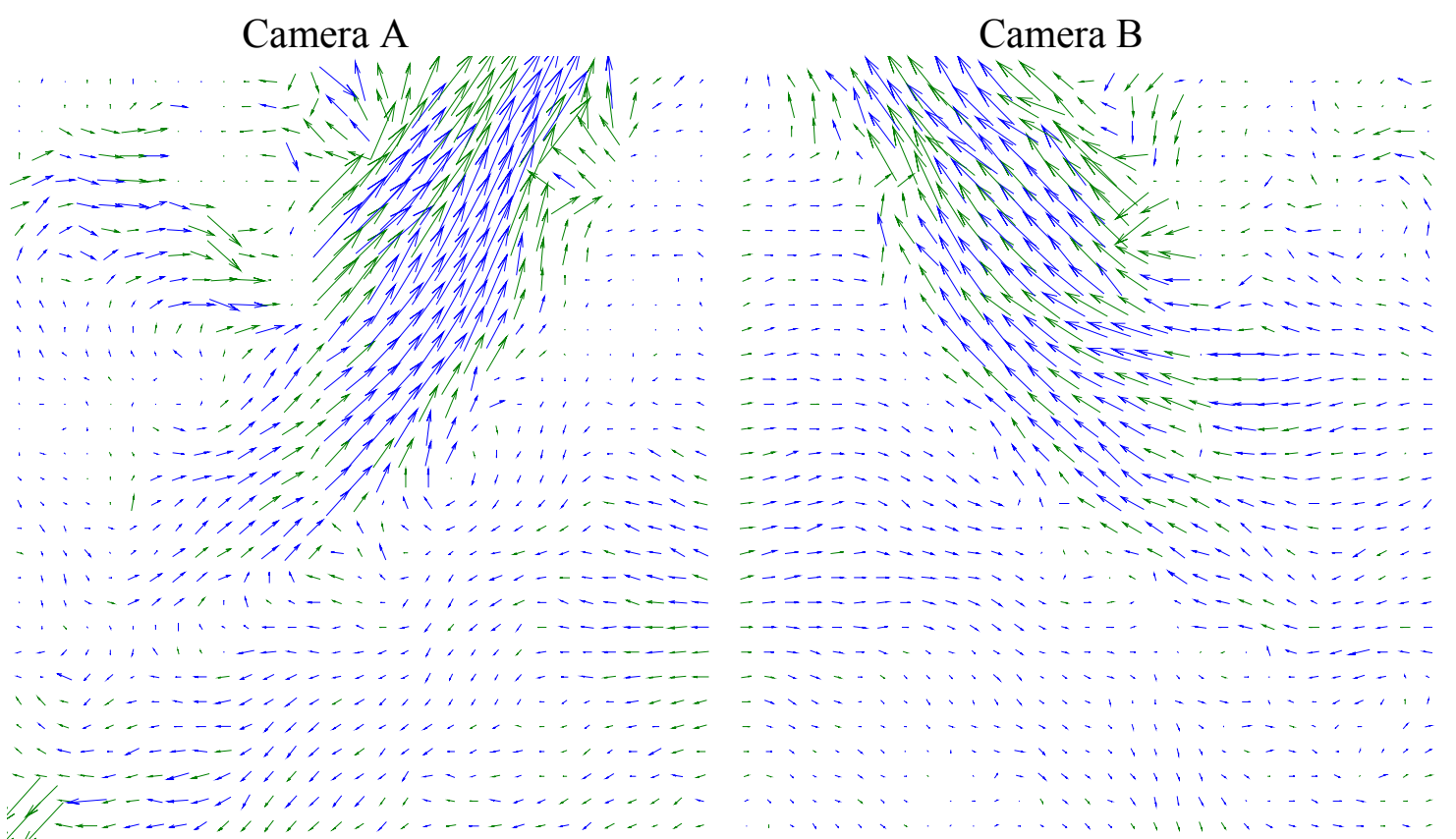

Figure 18 Displacement vectors computed from Figure 16 images A and B using the adaptive cross correlation technique.

The displacement maps, $\Delta d$, in Figure 18, which will from now on be referred to as adaptive correlations, were further processed by applying the stereoscopic calibration function to map the displacements recorded in two dimensions to represent displacement vectors in the three dimensional object space. The result of applying the stereoscopic calibration is shown in Figure 19. The displacements were simply divided by the time between the laser pulses to compute the velocity components. This $\Delta t$ is stored in the header information with each set of images. The vectors in Figure 19 represent the $v_{x}$ and $v_{y}$ velocity components while the color map represents the velocity component normal to the plane, $v_{z}$.

Validation and substitution information was retained with each vector. Adaptive correlations, Figure 18, did not contain rejected vectors, only substituted vectors. The vector results in Figure 19 retain the status of the vectors from the adaptive correlations as either valid or valid and substituted. Retaining this status flag is important for computing vector statistics. 


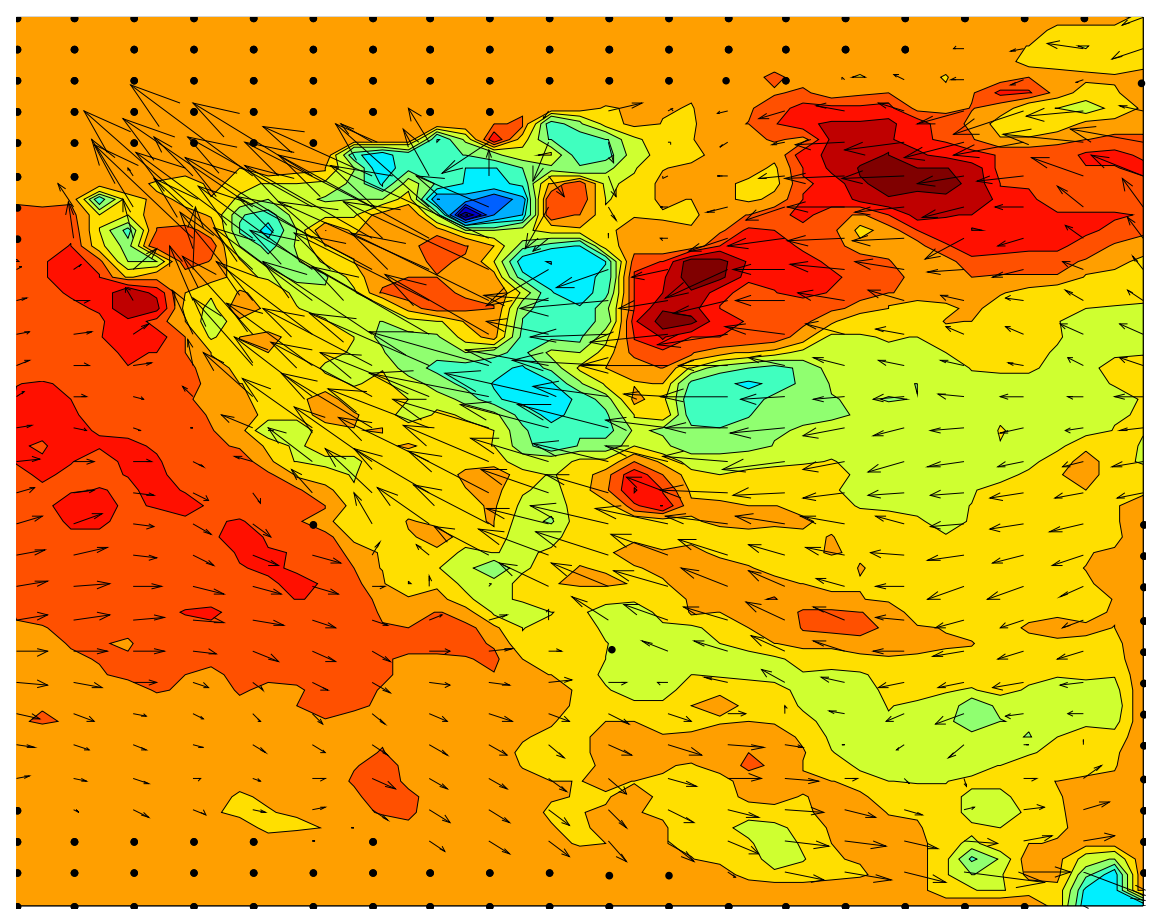

Figure 19 Velocity vector field computed from displacements. Vectors represent $v_{x}$ and $v_{y}$ components, color map represents $v_{z}$ component.

All of the vector processing tasks previously described were standard functions of the Dantec Dynamics PIV software. Computation time for standard cross correlations and stereoscopic transformations were on the order of one second or less. The time to compute a single adaptive correlation was about 6-10 seconds for the recipe applied in this study. Therefore for a single experiment which included a set of 60 measurements, requiring 120 adaptive correlations, the computation time could range from 12 minutes to 20 minutes.

\subsubsection{Mean Vector Maps}

At least 60 instantaneous vector maps were recorded at each measurement location. The stack of 60 vector maps represents a time ensemble of measurements for a single steady state experiment. The mean vector map is therefore a time average computed at each $(x$, $y$ ) coordinate. However, at each time $t$ and each coordinate position $(x, y)$ a valid vector was not necessarily computed. For example, at the $z=0 \mathrm{~cm}$ location and for coordinate position $(3.7 \mathrm{~mm}, 617.5 \mathrm{~mm})$ only 18 valid vectors were computed from the 60 measurements. Therefore the average vector is for a set of 18 vectors and not 60 . In this case the statistics were computed for valid vectors only. Statistics were also computed for valid and substituted vectors, which meant that a set of 60 vectors were available because the substituted vectors were being included. The statistics were computed with the PIV software which also included other statistical values such as standard deviation, and co-variance of the velocity components. 


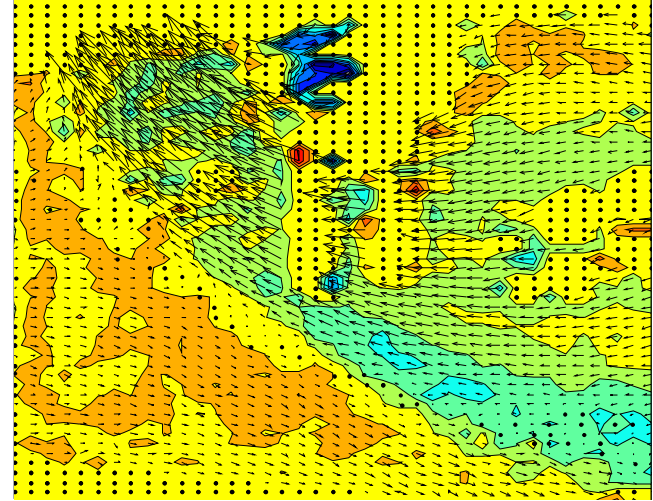

Valid vectors

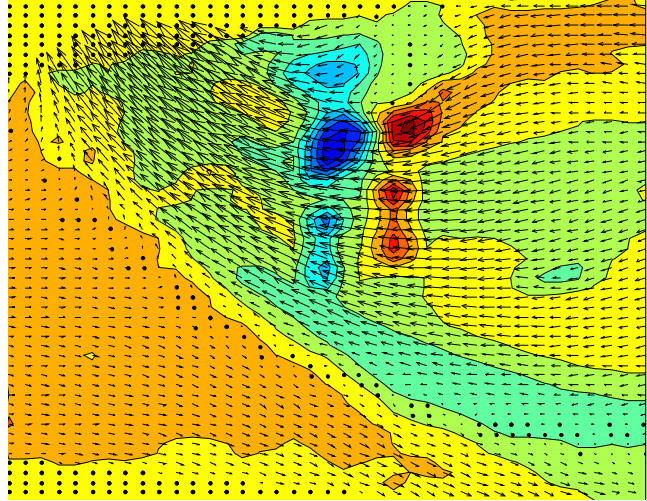

Valid and substituted vectors

Figure 20 Example of mean vector maps for two cases of vector statistics.

Examples of mean vector maps are displayed in Figure 20. The example of the vector statistics computed with only valid vectors is on the left. There were regions without valid vectors and these regions are displayed with dots overlaying the color map (note that the color map is displayed for the $v_{z}$ component in this region but this is an artifact of the graphics program). These regions correspond to saturated pixel areas in the image maps, usually due to reflections from the laser sheet on the enclosure walls. Therefore it is recommended to avoid reflections in the camera field of view which will cause a constant degradation to the available information.

The vector map on the right in Figure 20 is an example of the vector statistics computed for both valid and substituted vectors. Vectors were interpolated from surrounding valid vectors and substituted into the regions with saturated pixels in each instantaneous vector map. The result is a smooth and continuous mean vector field. The field of mean valid only vectors is spotty or lacks data in some very important regions. In order to make quantitative computations such as mass flow rate the data would need to be interpolated through these regions. The valid and substituted vector field is essentially a two dimensional interpolation therefore the mean vector field computed from valid and substituted vectors is used for both the qualitative display of information and quantitative computations.

\subsubsection{Vector Map Vertical Overlap}

The camera orientation for this set of experiments resulted in a vector field covering 0.71 $\mathrm{m} \times 0.62 \mathrm{~m}(l \times h)$. The dimensions of the enclosure doorway opening were $0.54 \mathrm{~m} \times 1.0$ $\mathrm{m}(w \times h)$. Due to space restrictions and the need for optimum scattering signals, the laser sheet passed through the doorway propagating perpendicular to the doorway plane while the cameras were oriented to record the forward scattering from the seed particles. Therefore the resulting vector field is a planar slice of the flow inside and outside the enclosure. The height of the vector field is $0.61 \mathrm{~m}$ and therefore the enclosure had to be 
translated in the vertical direction to map the entire vertical vector field. This was performed in three stages. As indicated in Figure 21, experiments were conducted in overlapping lower, middle, and upper regions of the doorway. While in a region, all $z$ location (normal to vector field plane) measurements were conducted. The enclosure was translated vertically and the experiments at the $z$ locations repeated. The vector maps in Figure 21 are mean vector maps at a $z=0 \mathrm{~cm}$. The vector maps statistics were computed for valid and substituted vectors.

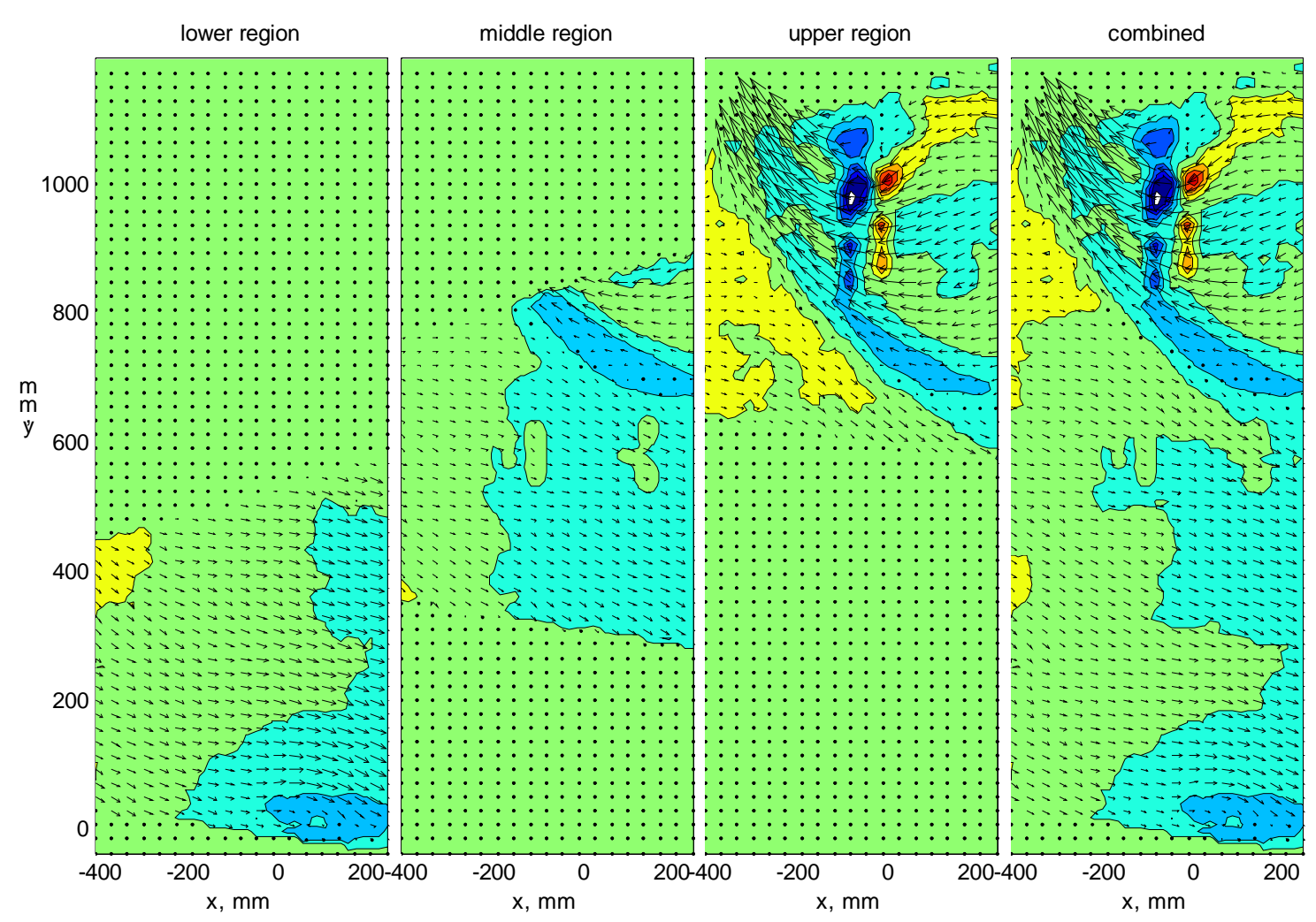

Figure 21 Overlapping vertical measurement regions.

There was intentional overlap in the vertical measurement regions in order to account for camera perspective and to provide some repeatability information. A composite mean vector map was created by layering the three mean vector maps and computing a second mean within the overlapping regions. The mean was weighted with respect to the number of valid vectors included in the statistics of the time averaged mean (set of 60 measurements). For example if two mean values, 10 and 20, were computed from sets of 50 and 10 measurements, respectively, the mean of 10 and 20 will be weighted more towards 10 because of the larger number of measurements. The vector map on the far right of Figure 21 is the resulting composite mean vector map for the entire vertical distance of the doorway. For clarity, every other vector is displayed. The doorway plane is located at $x=-14 \mathrm{~mm}$ and the vertical distance of the doorway ranges from $y=40 \mathrm{~mm}$ to $1040 \mathrm{~mm}$. 
Vertical slice measurements were taken at $5 \mathrm{~cm}$ intervals across the doorway plane, with $z=0 \mathrm{~cm}$ representing the center of the doorway. The result was 11 composite vector maps similar to the one in Figure 21. The 11 vertical z-slice locations are shown as dotted lines in Figure 22. The extent of the vector field measurement is shown as the dotted box in Figure 22. The resulting measurement field is a volume of $0.67 \mathrm{~m} \mathrm{x} 1.0 \mathrm{~m}$ x $0.5 \mathrm{~m}(l \times h \times w)$.
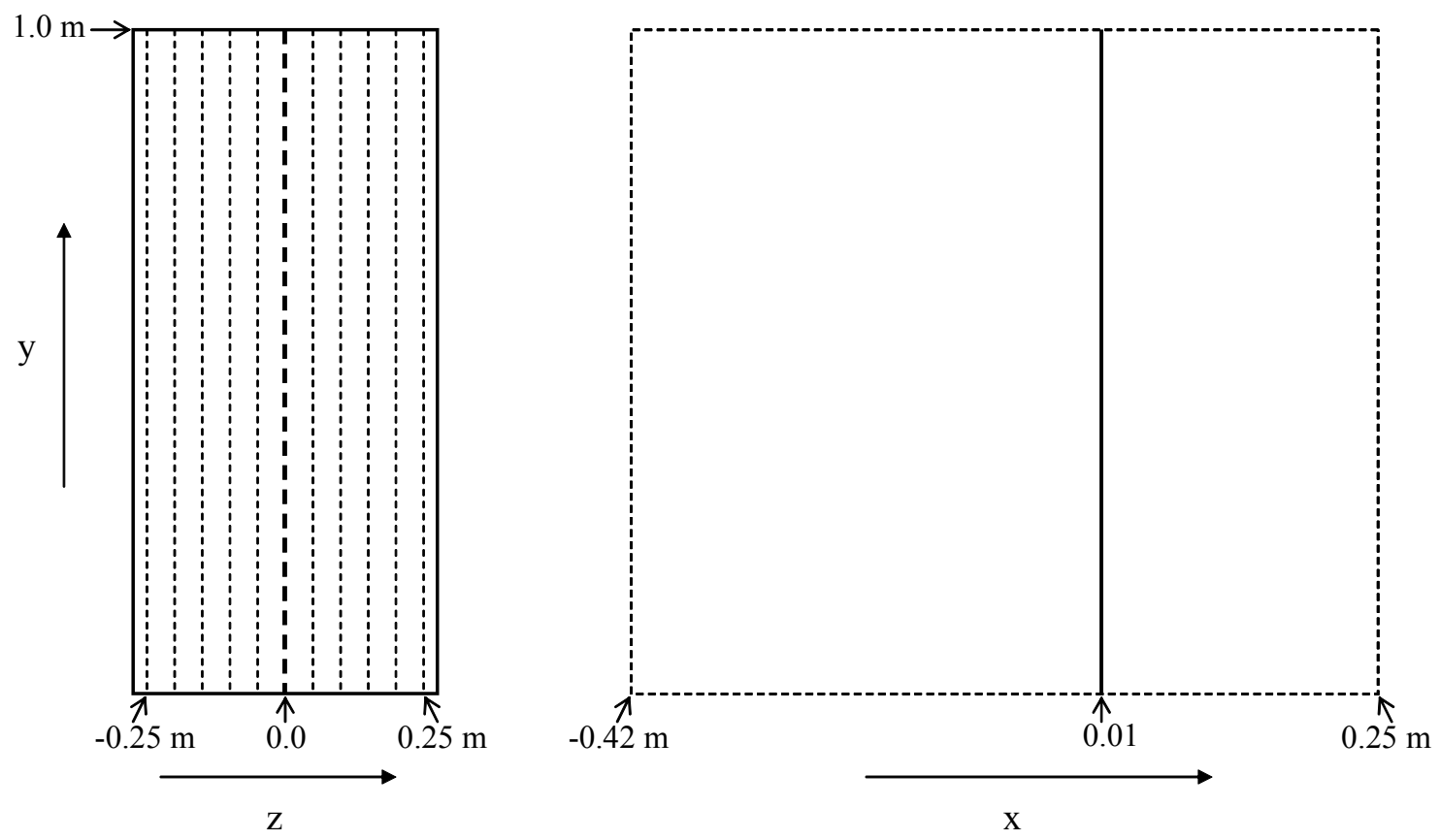

Figure 22 Doorway (solid line), measurement slice planes (dotted line).

\subsection{Flow Visualizations}

\subsubsection{Velocity Scalar Field}

Imaging measurements taken at sequential locations generate a volumetric field of information. By mapping an entire measurement volume, the uncertainty associated with assuming that a specific measurement profile applies across a region is reduced. In addition, volume mapping offers visualization and qualitative information of the flow field that is difficult to achieve with limited point measurements. Thirty three measurement slices were conducted in this study and a sample of the resulting volumetric visualization information is shown in Figure 23. The figure offers a visual representation of the velocity magnitude within the vicinity of the doorway. That vicinity includes the doorway plane and a finite amount of space on both sides of the plane. The upper and lower layer flows are clearly distinguishable; the upper layer flow having a negative sign convention for flow exiting the enclosure. It is apparent that the lower layer flow moves at a much slower velocity compared to the fastest upper layer flow which moves as fast as $1.5 \mathrm{~m} / \mathrm{s}$. 


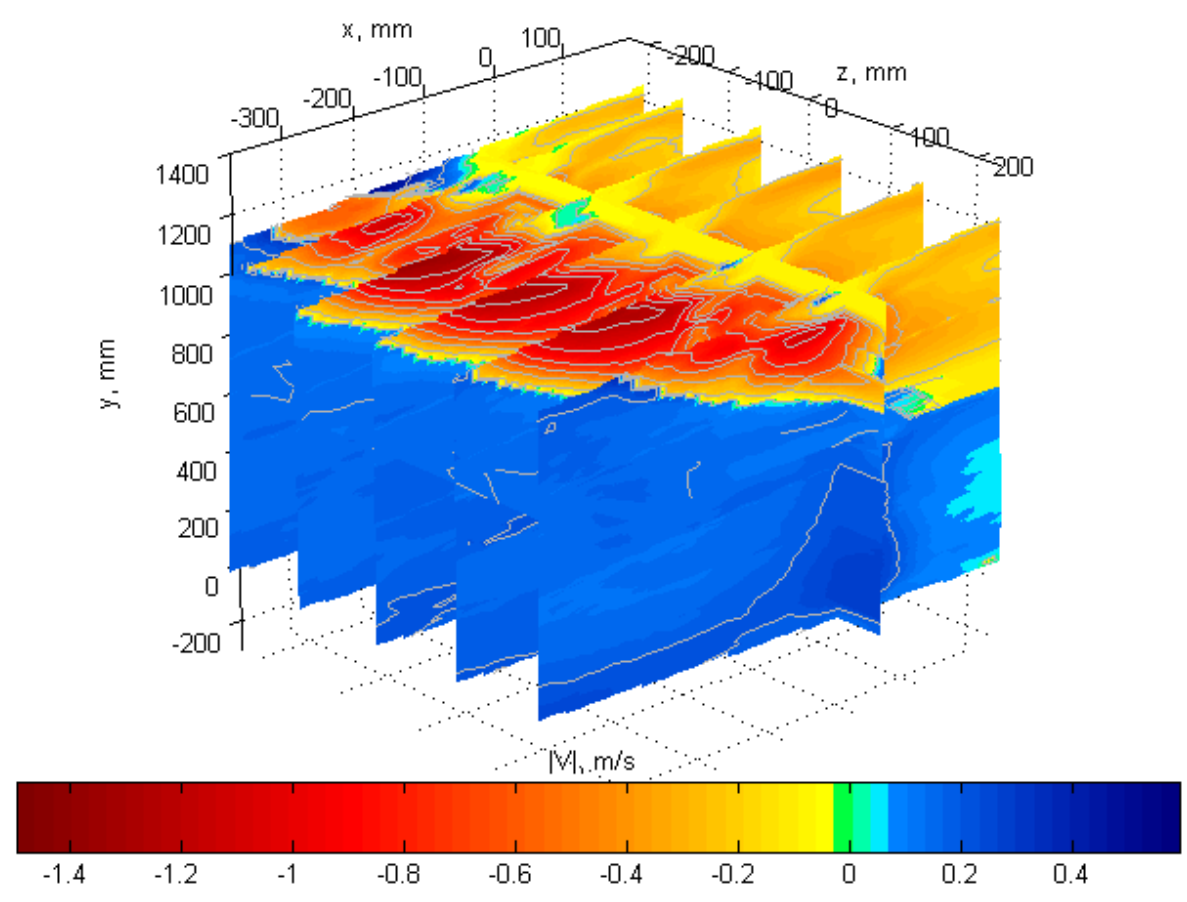

Figure 23 Velocity magnitude volume map.

A vertical profile of velocity measurements through the center of the doorway has often been used to graphically convey the concept of a fire induced doorway flow. In a likewise manner, Figure 24 displays a planar field of the velocity measurements through the center of the doorway. The image displays flow in the ambient, just outside of the doorway (located at $x=-14 \mathrm{~mm}$ ), and the flow immediately inside the enclosure. Velocity magnitude is a positive quantity, however a sign convention is necessary to properly display the counter current flows. The direction of the flow is determined by the $v_{x}$ component of the velocity vector. Therefore the $v_{x}$ velocity field was normalized by its absolute value and the product of this ratio and the velocity magnitude field results in a velocity magnitude field with directional information, Equation 28 . This sign convention is with respect to the bulk flow only, $v_{x}$, which is into and out of the enclosure.

$$
|V|_{ \pm}=|V| \frac{v_{x}}{a b s\left(v_{x}\right)}
$$




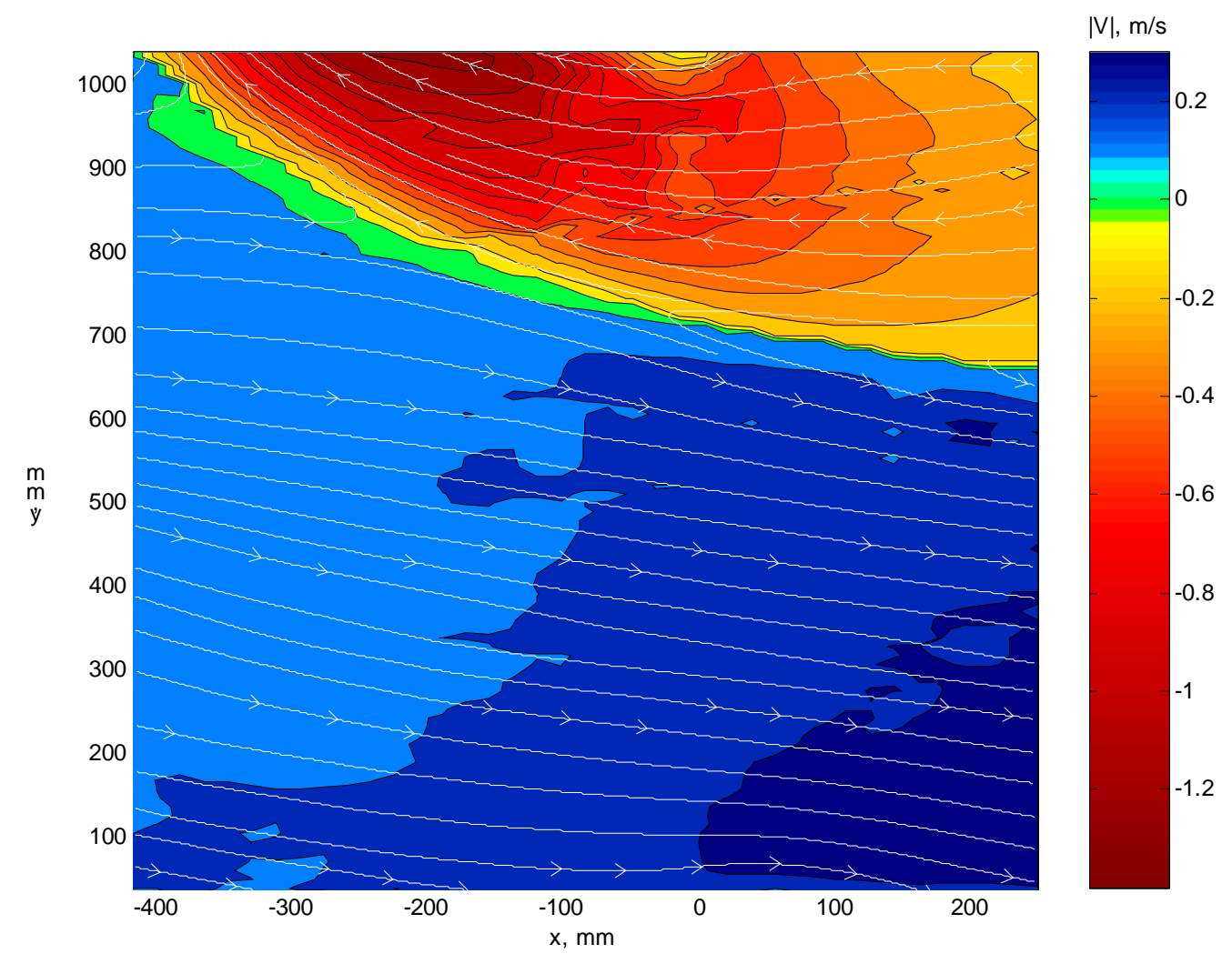

Figure 24 Vertical slice of velocity magnitude and streamlines at $z=0 \mathrm{~mm}$. Doorway plane located at $x=-14 \mathrm{~mm}$.

Figure 24 is a quantitative representation of what has been conveyed in words and sketches in the past. The ambient air outside the enclosure is sucked (left to right) into the enclosure. The flow induced in the ambient is slow and gradual along the flow direction and the vertical change in flow speed is very gradual. The helium-air mixture (low density) flow out of the enclosure (right to left) is faster and far less gradual. Distinct flow gradients are evidenced by the acceleration of the flow out of the enclosure and by the deceleration of the flow at the layer interface. This image also shows the complete shape and location of the layer interface in the vicinity of the doorway. The location of the neutral plane, defined by zero velocity, is accurately determined from data fields such as this and will be discussed later.

It should be noted that the fast and accelerating flow at the top left of the image is mostly due to the buoyancy of the low density helium-air mixture. However, an exhaust hood was placed above the enclosure and over the exit to exhaust the large amounts of helium being released in order to prevent asphyxiation. Therefore some fraction of the upward velocity outside the room may be due to the exhaust system. 


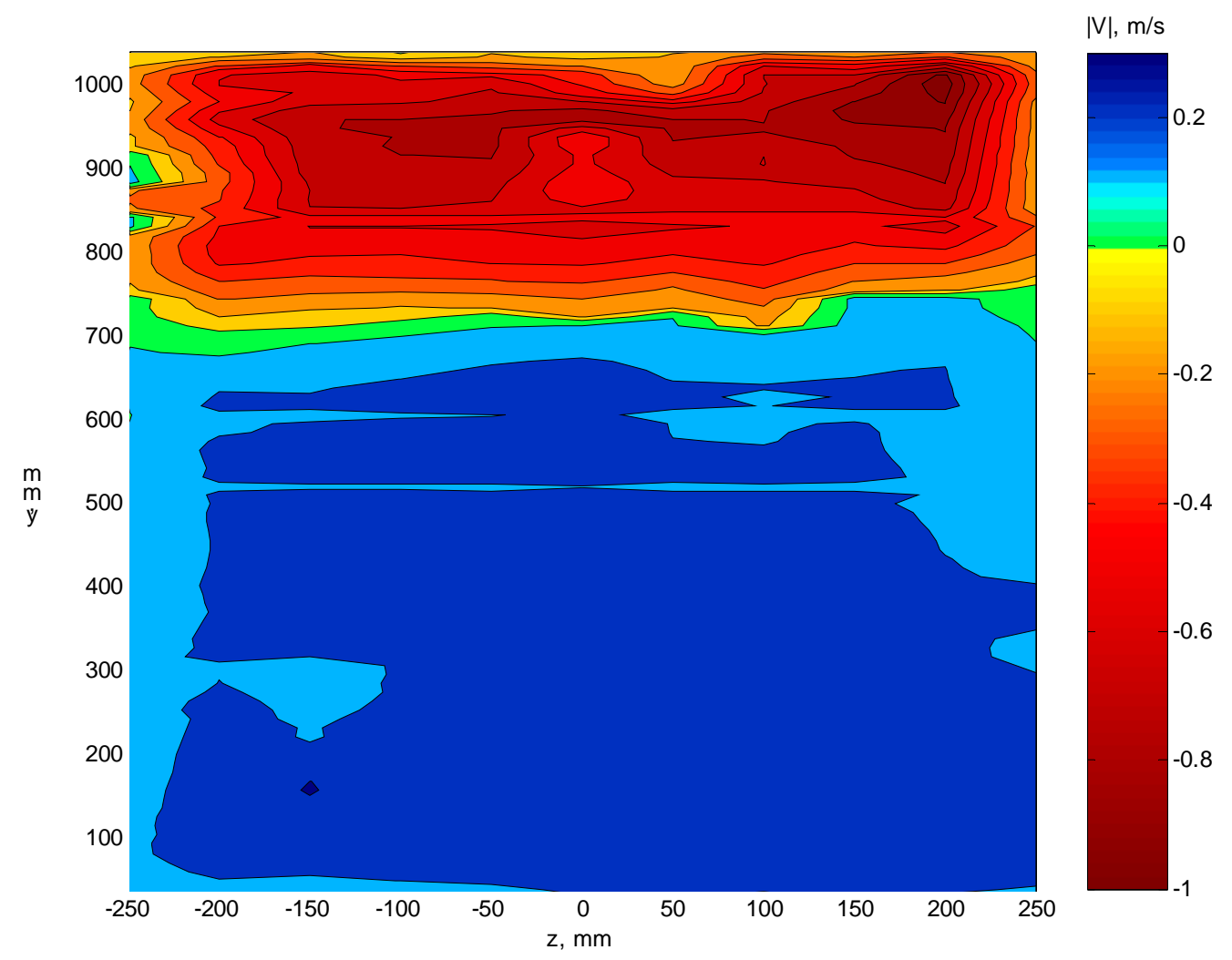

Figure 25 Velocity magnitude vertical slice across the doorway plane, $x=-14 \mathrm{~mm}$.

A cross section of the velocity data at the doorway plane is presented in Figure 25 from the point of view of the observer standing outside the enclosure looking into it. Recall that measurements were conducted at every $5 \mathrm{~cm}$ in the $z$ direction. The measurement spacing in the $y$ direction was $1.1 \mathrm{~cm}$. The color contour plot shown interpolates the color map between measurement locations. Therefore the velocity information in the $z$ direction is largely estimates (except for the $11 \mathrm{z}$ locations) and should be viewed as such, especially in regions of high gradients.

The neutral plane height slightly increases from left to right across the doorway. The increase is less than $2 \mathrm{~cm}$. A possible reason for the increase is that the flow into the enclosure was not symmetric due to flow currents within the laboratory. Another possible reason could have been unbalanced suction from the exhaust hood above. The neutral plane occurs at an average height of $730 \mathrm{~mm}$ across the doorway. This was computed by determining the two locations along a vertical line where the $v_{x}$ velocity is very low and changes sign; one location will be for positive $v_{x}$ and the other will be for negative $v_{x}$. This resulted in $22 y$ locations, two for each $z$ position. The geometric mean of these values was then computed. The image shows that flow into the enclosure occurs over $72 \%$ of the doorway area while flow out of the enclosure occurs over the remainder. 
There is a relatively uniform velocity distribution in the lower layer of the doorway (inward flow), with greater uniformity in the $z$ direction. The upper layer velocity (outward flow) is not uniform in the $y$ direction but for the portion just above the layer interface it appears to be quite uniform in the $z$ direction.

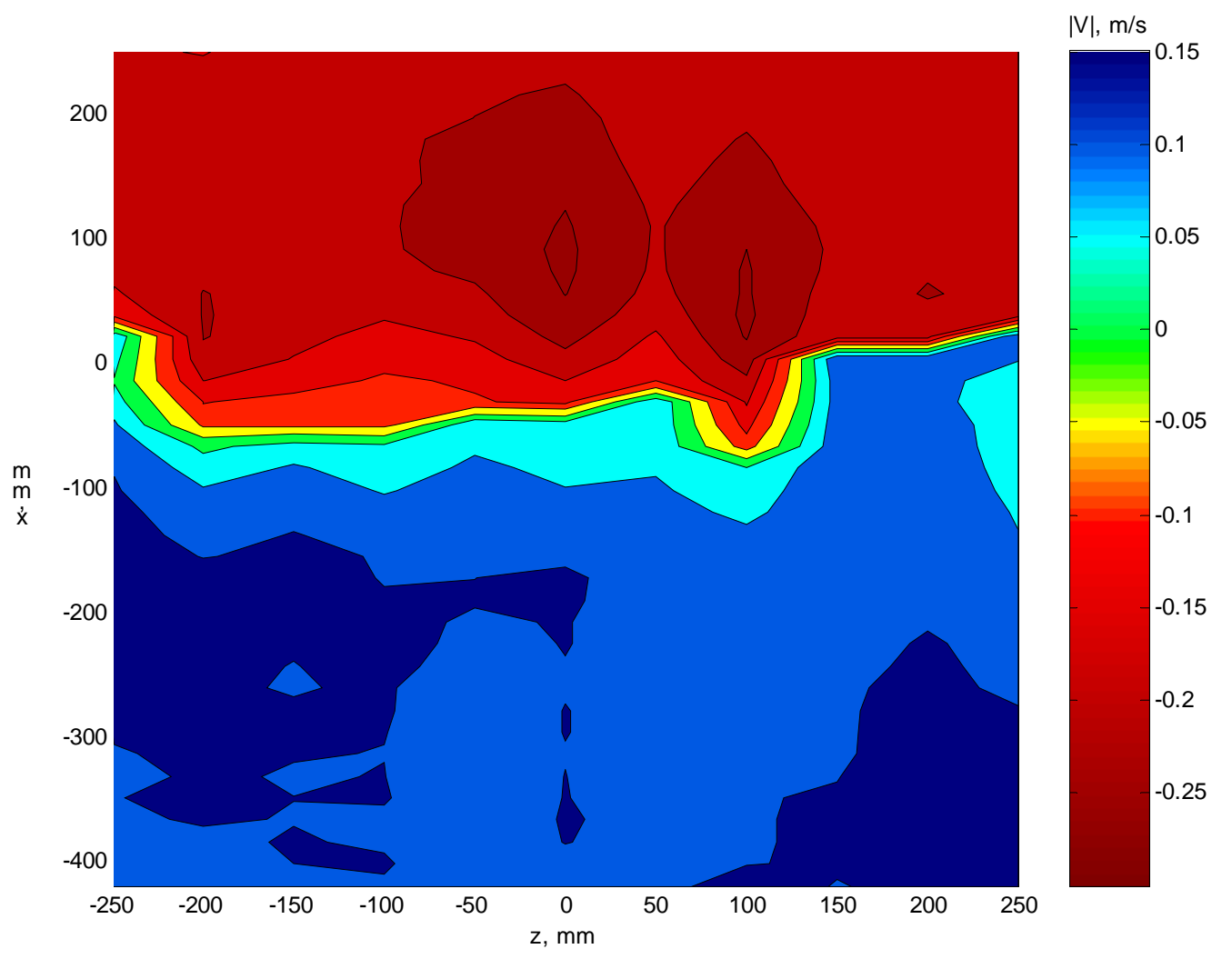

Figure 26 Horizontal slice of velocity magnitude at $y=730 \mathrm{~mm}$.

A bird's eye view of the velocity magnitude field is displayed in Figure 26. The image is a horizontal slice at the mean height of the neutral plane, $y=730 \mathrm{~mm}$. The doorway plane was located at $x=-14 \mathrm{~mm}$, therefore flow both inside and outside the enclosure is displayed. Identical to Figure 25, the color map is interpolated between measurement locations. Measurement spacing in the $x$ direction is $1.8 \mathrm{~cm}$ and $5 \mathrm{~cm}$ in the $z$ direction. Recall that positive velocity is flow into the enclosure while negative velocity is flow exiting the enclosure. Similar to Figure 25, this view shows the increase in flow gradients in the vicinity of the layer interface.

\subsubsection{Velocity Vector Field}

It is often easier to display visual information of a scalar quantity, therefore the velocity magnitude was displayed in the previous figures, Figure 23 through Figure 26. However, a wealth of information within the scalar quantity remains to be explored. It is possible 
to display the information for each velocity component as demonstrated in Figure 27. Each velocity component is displayed for a vertical slice through the doorway. The color map represents the $v_{z}$ velocity component which is normal to the plane of the page and the vectors represent the $v_{x}$ and $v_{y}$ velocity components. The doorway plane is located at $x=-14 \mathrm{~mm}$, so this image is an example of the full velocity vector field for a region inside and outside the enclosure.

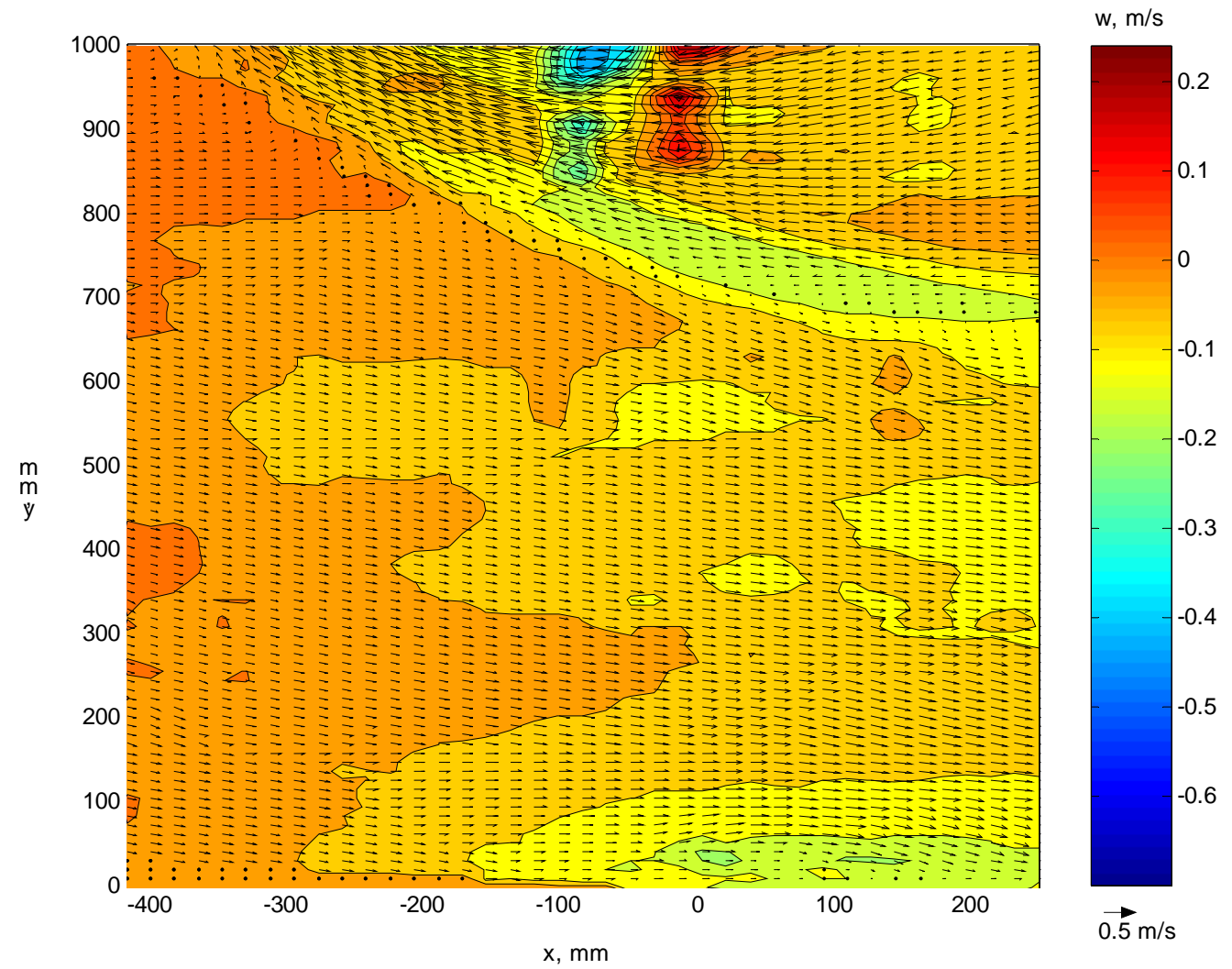

Figure 27 Vertical velocity slice through doorway centerline, $z=\mathbf{0 ~ m m}$. Color map represents $v_{z}$ velocity component, vectors represent $v_{x}$ and $v_{y}$ velocity components. The doorway plane is located at $x=-14 \mathbf{~ m m}$. 


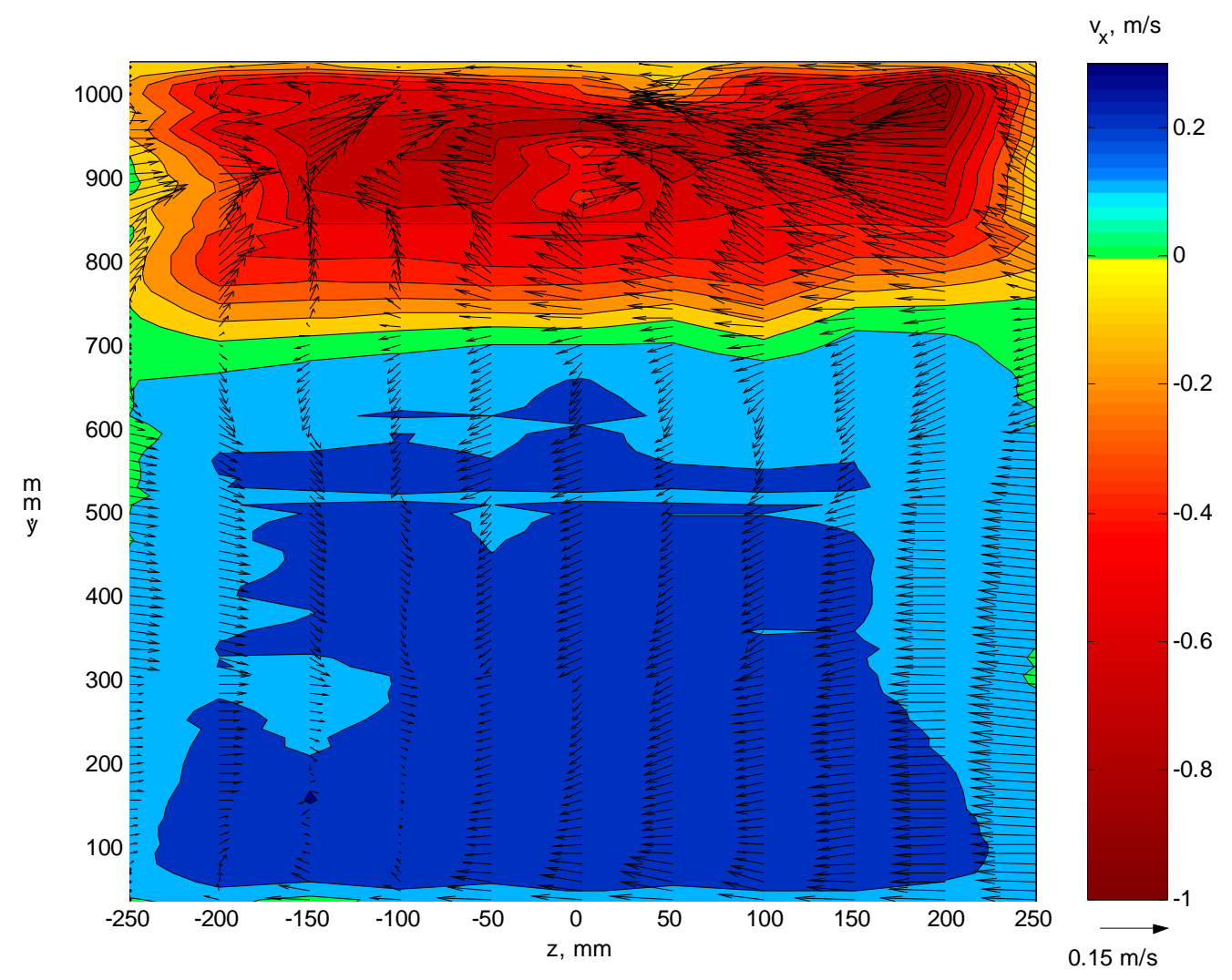

Figure 28 Vertical velocity slice across doorway plane, $x=-14 \mathrm{~mm}$. Color map represents $v_{x}$ velocity component, vectors represent $v_{y}$ and $v_{z}$ velocity components. The neutral plane lies within the green contour which ranges from (-0.05 to 0.05$)$ $\mathbf{m} / \mathbf{s}$.

Figure 28 is a vertical slice of the velocity vector field across the doorway plane. The color map represents the $v_{x}$ velocity component which is now normal to the plane of the page. The vectors represent the $v_{y}$ and $v_{z}$ velocity components parallel to the plane of the page. Figure 25 and Figure 28 have very similar color maps, therefore indicating that displaying the scalar quantity was effective in conveying much of the qualitative information for this specific slice view. Figure 28 demonstrates that, at the doorway plane, there can be portions of the flow field where the $v_{x}$ velocity component does not dominate or where it is on the same order as the $v_{z}$ velocity component. This observation is generally accepted but has not been adequately quantified for fire induced doorway flows due to the difficulty of conducting multi-component velocity measurements over large regions of interest. The availability of stereoscopic PIV measurements now increases the capability to better understand the structure and dynamics of such flows. 


\subsection{Gas Density}

In order to compute the mass flow rate through the doorway the gas density field in the doorway plane was also determined, as required by Equations 1 and 2. Since the fluid flow was non-reacting, the three gas species (oxygen, nitrogen, and helium) were conserved, and the ratio of oxygen volume fraction to nitrogen volume fraction is constant in ambient air, it was necessary to measure the volume fraction of only one gas constituent to infer the gas density field. Oxygen volume fraction, $X_{\mathrm{O}_{2}}$, was measured at a variety of elevations at the center of the doorway plane, $z=0 \mathrm{~mm}$. The experiment was allowed to run long enough to establish steady state conditions, therefore average oxygen volume fraction was computed from the time history results.

Selected time history plots of oxygen volume fraction are shown in Figure 29. Gas sampling was continuous and the helium flow was turned on 60 seconds after data acquisition began. The helium flow was maintained for 180 seconds and then shut off. Data acquisition was continued until the oxygen volume fraction returned to the ambient value. Figure 29 demonstrates the growth of the layer of helium mixed with air. At the lower elevations it takes longer for the helium-air mixture to descend.

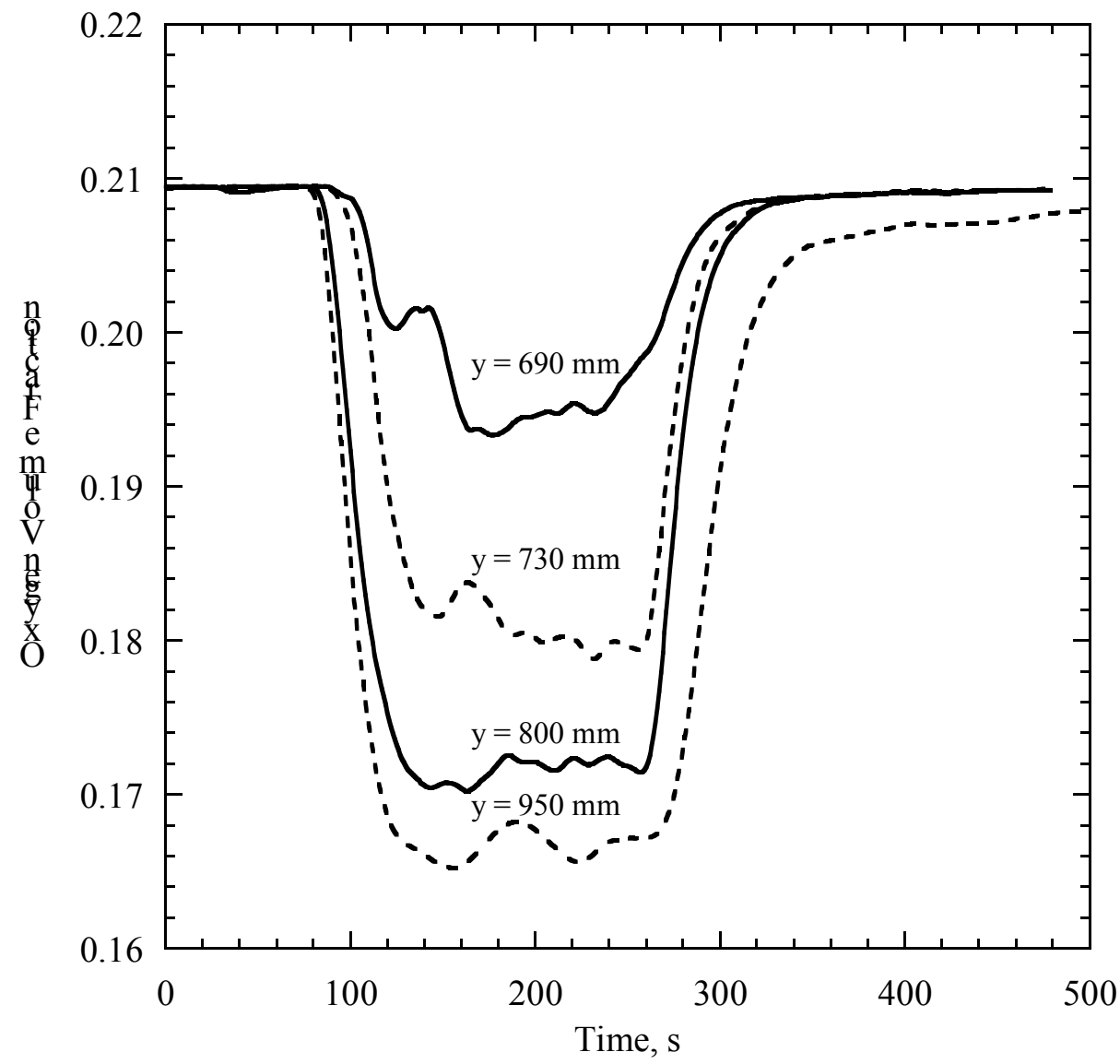

Figure 29 Time history plot of oxygen volume fraction measured at selected elevations at the centerline of the doorway plane. 
Average oxygen volume fraction is determined by integrating the time history results over the period of assumed steady state conditions. This period is defined by the full width at half the deflection height of the curve and the average oxygen volume fraction is given by the following relation.

$$
X_{O_{2}, a v g}=\frac{\int_{t_{1}}^{t_{2}} X_{O_{2}}(t) d t}{t_{2}-t_{1}}
$$

The mean volume fractions of nitrogen and helium are defined in terms of the mean oxygen volume fraction using the following relations, $X_{N_{2} \text {,avg }}=3.773 X_{\mathrm{O}_{2} \text {,avg }}$ and $X_{\mathrm{He}, \text { avg }}=1-4.773 X_{\mathrm{O}_{2}, \text { avg }}$. The gas mixture is assumed to be an ideal gas mixture with invariant temperature throughout and the variation in pressure negligible with respect to its effect on gas density. Therefore the molecular weight, $M W_{m i x}$, is the only variable in the equation of state and is given in terms of the mean oxygen volume fraction.

$$
M W_{\text {mix }}=X_{\mathrm{O}_{2}, a v g}\left(M W_{\mathrm{O}_{2}}+3.773 M W_{N_{2}}-4.773 M W_{\mathrm{He}}\right)+M W_{\mathrm{He}}
$$

Following the assumptions that the gas mixture behaves as an ideal gas and temperature and pressure are invariant, the gas density is defined with respect to the gas density of air at standard temperature and pressure $(300 \mathrm{~K}$ and $101.325 \mathrm{kPa})$.

$$
\rho_{\text {mix }}=\rho_{\text {air }, \text { STP }} \frac{M W_{\text {mix }}}{M W_{\text {air }}}
$$

The vertical profile of gas density is shown in Figure 30. Measurements were conducted in the full vertical extent of the outflow region and for a portion of the inflow region where $X_{\mathrm{O}_{2} \text {,avg }} \leq X_{\mathrm{O}_{2}, a m b}$. Measurements therefore extended from $550 \mathrm{~mm}$ to $950 \mathrm{~mm}$ in the y direction. Multiple measurements were conducted at a few locations and an interpolation of the mean values was computed as shown Figure 30 . Below $550 \mathrm{~mm}$, the gas flowing into the enclosure is purely air. The gas density field is approximated from the interpolated vertical profile, $\rho(y, z) \approx \rho_{\text {mix }}(y, 0)$. This approximation is valid since measurements of gas density in the outflow region and along a line at constant elevation varied by $1 \%$ or less. 


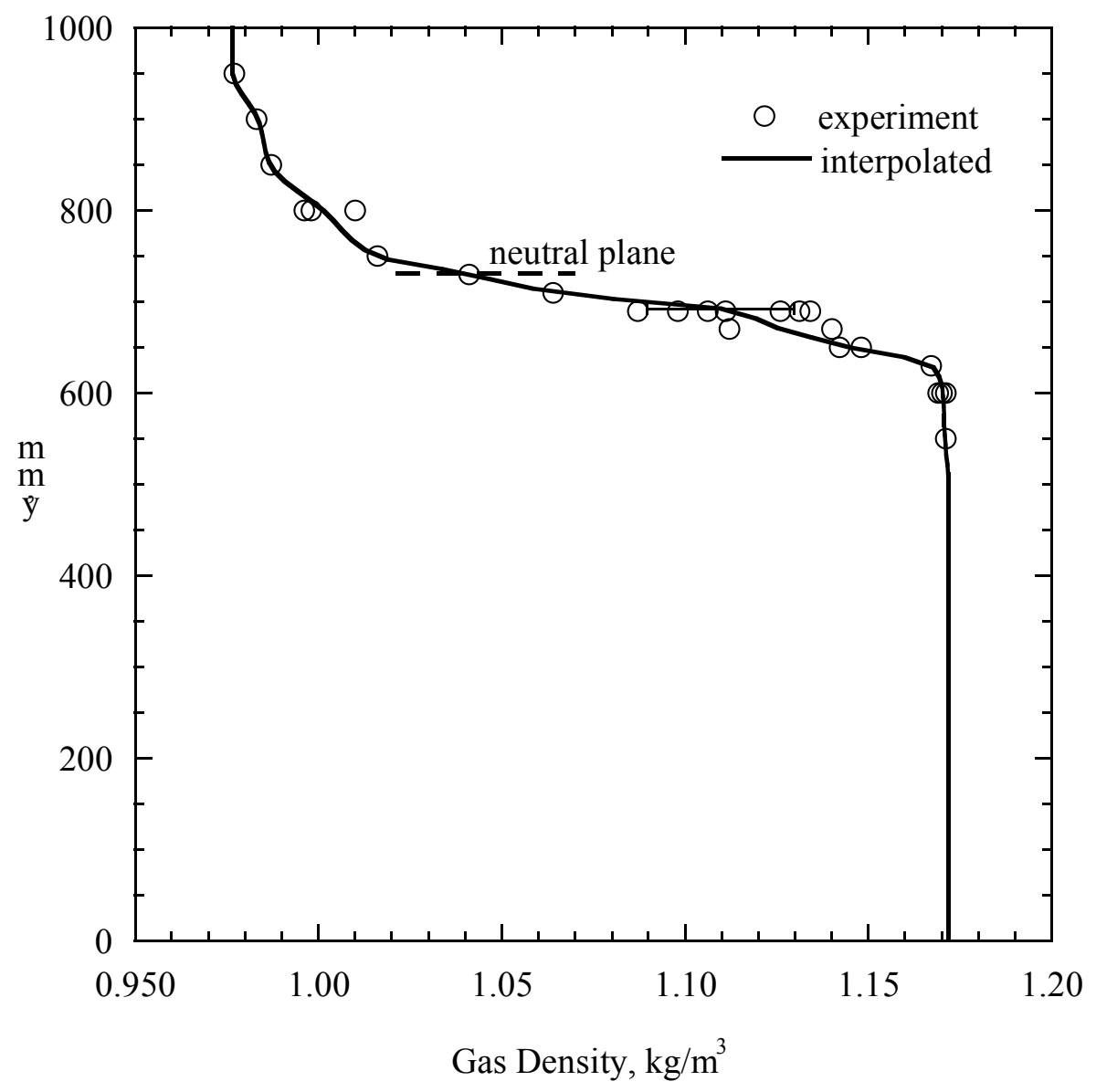

Figure 30 Vertical profile of gas density measured at $z=0 \mathbf{~ m m}$.

Note that the mean neutral plane height is $730 \mathrm{~mm}$ and there is a measurable oxygen deficit for the gas flowing into the enclosure between $550 \mathrm{~mm}$ and $730 \mathrm{~mm}$. The neutral plane location varies throughout the experiment and therefore it is expected that there will be a mean oxygen deficit below the neutral plane. However other causes of the oxygen deficit must be given consideration such as the flow mixing that occurs at the interface. Simultaneous measurements of concentration and velocity with comparable time response would offer more information to interpret these mean results.

\subsection{Mass Flow Rate}

Typical measurements of fire-induced flow in a doorway are bi-directional probe measurements. The assumption is made that the measurement is dominated by the $v_{x}$ velocity component, the component normal to the doorway plane. This component of velocity is used to compute the net mass flow rate of gas through the doorway. A goal of this work is to determine how much of a discrepancy exists between mass flow rate computations based on velocity magnitude (flow speed) and computations based on the $v_{x}$ component of the velocity vector. Figure 31 is a vertical slice of the ratio of the square of the $v_{x}$ velocity component and the square of the velocity magnitude at the doorway plane, 
$v_{x}^{2} /|V|^{2}$. The three levels of red represent ratios of $0.7,0.8$, and 0.9 with 0.9 being the darkest shade of red. The figure demonstrates that for most of the flow region the contribution to the velocity magnitude by $v_{x}$ is $70 \%$ or better. However, a $90 \%$ contribution by $v_{x}$ occurs in less than $1 / 3$ of the flow region. This study estimates the discrepancy between mass flow rate computations based on the directly measured $v_{x}$ velocity component and the velocity magnitude.

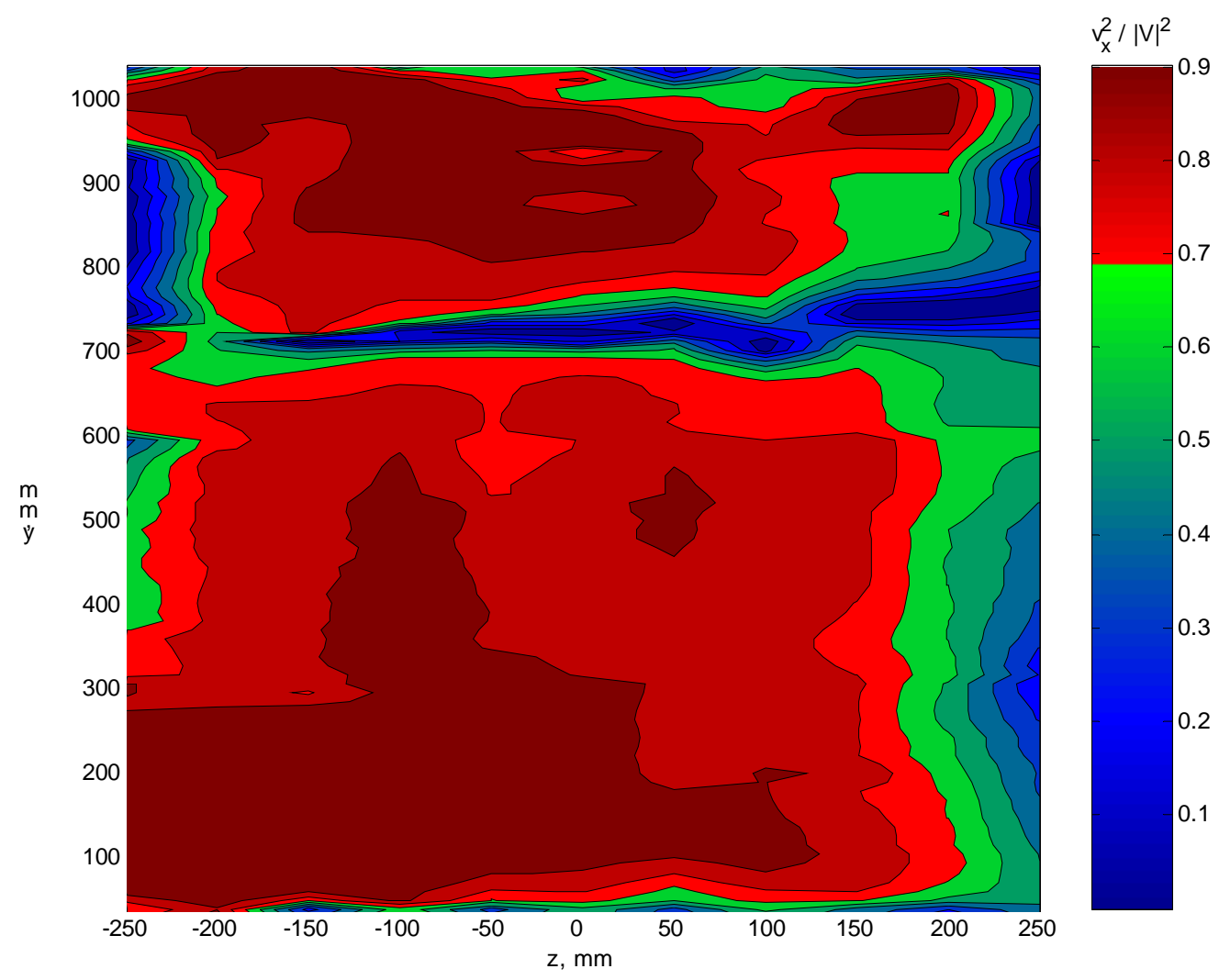

Figure 31 Vertical slice at doorway plane, $x=-14 \mathrm{~mm}$, of $v_{x}{ }^{2}$ normalized by $|V|^{2}$.

A vertical slice of the mean velocity field in the entire doorway plane is shown in Figure 28. Flow normal to the doorway plane, $v_{x}$, is displayed as a color field. The locations where the flow changes direction lie within the green contour. The neutral plane is relatively uniform across the doorway, with an average height of $730 \mathrm{~mm}$. Along with the physical boundaries of the doorway, the neutral plane location defines the limits of integration for computing gas mass flow rate into the enclosure.

Ideal mass flow rate entering and exiting the enclosure was described by Equations 1 and 2. The gas density field is approximated from a single vertical profile of gas density measured at the center of the doorway, $\rho(y, z) \approx \rho_{\text {mix }}(y, 0)$, as described in Section 6.3. Since the full density field cannot be measured at present, the experimental mass flow rates entering and exiting the enclosure are described by the following relations, where $W$ 
is the width of the doorway, $h_{N}$ is the height of the neutral plane, and $H$ is the height of the doorway.

Experimental mass flow rate in:

$$
\dot{m}_{i n, \exp }=\int_{-W / 2}^{W / 2} \int_{y_{o}}^{h_{N}} \rho(y) v_{x}(y, z) d y d z
$$

Experimental mass flow rate out:

$$
\dot{m}_{\text {out,exp }}=\int_{-W / 2}^{W / 2} \int_{h_{N}}^{H} \rho(y) v_{x}(y, z) d y d z
$$

A numerical integration using the trapezoid rule was applied to estimate the integrals described in Equations 1 and 2. The integration was performed in two parts. First the mass flux along a vertical line, $\int \rho(y) v_{x}\left(y, z_{i}\right) d y$, was computed at each of the eleven measurement locations across the doorway, $z_{i}$. Zero velocity was prescribed at each horizontal solid boundary. At the neutral plane, the velocity in either direction was approximately zero. The vertical spacing of the numerical integration, $d y$, was $10.7 \mathrm{~mm}$. Therefore the measured velocity value on the respective side of the interface was used in the integration and $h_{N}$ at each $z$ location, not the average $h_{N}$, was used to define the vertical integration limits. The horizontal spacing for the integration, $d z$, was prescribed as $10 \mathrm{~mm}$ in order to include the boundaries in the numerical integration. This required the array of line mass fluxes previously computed to be interpolated for a $10 \mathrm{~mm}$ spacing. Again, zero velocity was prescribed at the vertical boundaries and the numerical integration was performed horizontally across the doorway on the line mass fluxes to compute the mass flow rate. An example computation of mass flow rate into the enclosure is presented in the Appendix.

For a steady-state system the flow of mass into the system equals the flow of mass out of the system. Considering the enclosure as a complete system and assuming that the experiments were allowed to reach steady state, the following relation applies.

$$
\underbrace{\dot{m}_{\text {in, exp }}|+| \dot{m}_{H e} \mid}_{\dot{m}_{\text {in }}}=\underbrace{\left|\dot{m}_{\text {out,exp }}\right|}_{\dot{m}_{\text {out }}}
$$

The average mass flow rate of helium injected into the plume, $\dot{m}_{\mathrm{He}}$, was measured to be $0.0030 \mathrm{~kg} / \mathrm{s}$ for all of the experiments. The mass flow rates entering and exiting the doorway were computed by numerical integration as described above, and they are listed under the columns labeled "Doorway" in the table below along with their expanded uncertainty. 
Table 8 Experimental mass flow rates.

\begin{tabular}{ccc}
\hline \hline Mass Flow Rate In, kg/s & Mass Flow Rate Out, $\mathbf{k g} / \mathbf{s}$ \\
\hline Helium Injection & Doorway & Doorway \\
$0.0030 \pm 0.0001$ & $0.079 \pm 0.005$ & $0.061 \pm 0.008$ \\
\hline \multirow{3}{*}{ Relative Discrepancy: } & $\frac{\dot{m}_{\text {in }}-\dot{m}_{\text {out }}}{\dot{m}_{\text {in }}}=0.256$ \\
\hline
\end{tabular}

There is a relative discrepancy of $26 \%$ between the in and out mass flow rates. In an attempt to understand the source of this discrepancy, a comparison was made between the mass flow rates of helium out of the doorway and helium injected into the enclosure. Using the gas sampling data to determine $X_{\mathrm{He}}(y)$ (See Section 6.3), the helium mass flow rate out of the enclosure was computed by performing a numerical integration for the following relation.

$$
\int_{-W / 2}^{W / 2} \int_{h_{N}}^{H} \rho_{H e} X_{H e}(y) v_{x}(y, z) d y d z
$$

Employing a constant helium density in Equation $35, \rho_{\mathrm{He}}=0.1625 \mathrm{~kg} / \mathrm{m}^{3}(300 \mathrm{~K}$ and $101.325 \mathrm{kPa}$ ), the mass flow rate of helium out of the enclosure was computed to be $0.0019 \mathrm{~kg} / \mathrm{s}$. This was $37 \%$ less than the amount of helium being injected into the enclosure. Since the measured helium flow out of the enclosure was less than what was measured to flow into the enclosure, and the total out-flow was less than the total in-flow, it seems logical that the out-flow measurement may be flawed. A plausible explanation is the zero biasing of the velocity results due to laser sheet reflections causing large regions of invalid vectors. Vectors were interpolated through these regions in order to estimate the data necessary to compute the mass flow rate and to produce a smooth vector field for display purposes. Another plausible explanation is the influence of the exhaust hood when vertically translating the enclosure in order to measure the entire flow field. A check to build confidence in this hypothesis has not been performed, but the likelihood has not been dismissed. It is recommended that the entire doorway flow be measured in a single experiment. If this cannot be accomplished it is recommended to translate the optics. The latter option will require a more sophisticated setup and possibly more setup time, but the reward should be better repeatability of the experiments. Since the lower layer velocity measurements did not require the same degree of interpolation as the upper layer measurements, greater confidence is placed in the lower layer velocity measurements and they will be used in the remaining discussion.

\subsubsection{Example Comparison}

Stereoscopic PIV measures the complete velocity vector and therefore allows for a comparison of flow speed, $|V|$, and the desired velocity component, $v_{x}$. This comparison 
has significance because the response of a bi-directional probe may be more characteristic of the flow speed instead of the on-axis velocity component. The angular sensitivity of bi-directional probes is $10 \%$ or less in the range of 0 to 40 deg for off-axis flow. [7] This suggests that the probe response is not very selective with respect to flow direction and therefore exhibits an averaged response to the flow which is characteristic of the flow speed. The assumption that accompanies any bi-directional probe measurement is that the major contribution of the probe response comes from the on-axis velocity component. Figure 28 and Figure 31 are good illustrations of how velocity magnitude may have significant contribution from velocity components other than the $v_{x}$ component. In areas of the flow such as near the solid boundaries, near the neutral plane and the outflow, other velocity components, especially $v_{z}$, are competitive contributors to the flow speed measurement.

Bi-directional probe measurements were not conducted in this set of experiments. However, since the flow speed information was available from the Stereoscopic PIV results, an example comparison of the methods of determining the velocity fields could be conducted. Assuming that a single vertical array of bi-directional probes were horizontally scanned across the doorway the resulting velocity field would be $|V(y, z)|$, following the ideal introduced here that a bi-directional probe responds to flow speed. An alternate assumption is that a single vertical array of bi-directional probes were placed on the horizontal centerline, $z=0$, of the doorway and the resulting profile estimates the entire flow field. This assumes that the velocity variation with respect to $z$ is negligible and therefore the resulting velocity field would be, $|V(y, z)| \approx|V(y)|$. The latter assumption is typical of doorway flow measurements and the former is characteristic of a historical series of steady state experiments. $[5,19]$

The best mass flow rate estimate is computed using the velocity component normal to the doorway plane, $v_{x}$. Mass flow rate estimates computed using the velocity magnitude are normalized by the best mass flow rate estimate and displayed in Table 9. These estimates are for the flow into the room. The results show that if mass flow rate were computed from velocity magnitude measurements scanning the doorway or on centerline of the doorway, the computation would over-predict the best estimate. These example results are consistent with the need for a mass flow coefficient to correct mass flow rates computed from bi-directional probe measurements.

Table 9 Comparison of mass flow rate estimates.

\begin{tabular}{cc}
\hline \hline Velocity Field & Normalized Mass Flow Rate \\
\hline$V_{x}(y, z)$ & 1.00 \\
$|V(y, z)|$ & 1.11 \\
$|V(y, z)|=|V(y, 0)|$ & 1.25 \\
\hline
\end{tabular}

These results demonstrate the need to perform a direct comparison of bi-directional probe measurements and PIV measurements for fire-induced flows. Such a comparison can add to the body of data of flow coefficients for doorway flows and improve the confidence in these empirically determined constants. 


\section{Concluding Remarks}

A reduced-scale analog of a full-scale fire experiment was studied for the purpose of studying the potential use of Stereoscopic Particle Image Velocimetry to measure fire induced flows through vents. The size of the experiment was such that it was physically manageable yet large enough to challenge the performance of an off-the-shelf PIV system and therefore serve as a practice scenario prior to conducting experiments in full-scale enclosure fires.

Successful measurements of the complete velocity vector, $v_{x}, v_{y}, v_{z}$, were achieved and a mapping of the velocity field in the region of the doorway was performed to produce a volumetric field of the velocity. The vector field data shows that the buoyancy induced flow through the doorway can be highly three dimensional. Regions of the flow may exist where the velocity component normal to the doorway, $v_{x}$, may not dominate the velocity vector but may be in competition with other components such as the $v_{z}$ component. This was observed near the solid vertical boundaries of the doorway. Therefore conventional fire measurement tools such as bi-directional probes may not be reliable when used in these regions of the flow.

Since the $v_{x}$ component of the velocity vector field was directly measured, it could be combined with a vertical profile of gas density measurements that were conducted on centerline of the doorway to estimate the mass flow rate through the doorway. The experiments were assumed to be at steady state; therefore the mass flow into the enclosure should equal the mass flow out of the enclosure. The relative discrepancy between the in and out mass flow rates was $26 \%$. The cause of the discrepancy is not clear but possible reasons include a zero bias of the outflow velocity measurements due to laser sheet reflections saturating regions of the particle images or a change in experiment conditions due to the influence of the exhaust hood during the vertical translation of the enclosure. Considering the complexity of the experiment and that a large but limited number of experiments were conducted to achieve a single mass flow rate value, the discrepancy between the in and out flow is very reasonable and quantifies the success of such first ever measurements.

An example comparison of mass flow rate computed using the $v_{x}$ velocity component field and the velocity magnitude field was performed. The velocity magnitude is representative of a bi-directional probe measurement since the probe measurement lacks velocity component selectivity. The comparison demonstrates that mass flow rate will be over predicted if computed using the velocity magnitude data by as much as $25 \%$. This is consistent with mass flow rate orifice coefficients for doorway flows which suggest that mass flow rate computed from bi-directional probe measurements will be $30 \%$ greater than the actual mass flow rate.

Stereoscopic PIV measurements were successfully demonstrated for a large-scale flow field with image regions of $0.71 \mathrm{~m} \times 0.62 \mathrm{~m}(1 \mathrm{x} \mathrm{h})$. Seed particles were selected which effectively follow the flow and produce enough scattered light to produce quality images 
for vector processing. Achieving quality PIV results for such large-scale flow fields is not entirely trivial due to the need to balance the requirements for image resolution, scattering signal, and flow tracking. Conducting measurements in full-scale fire experiments will require image regions of $1 \mathrm{~m} \times 1 \mathrm{~m}$ to be imaged. Achieving the necessary requirements for quality PIV results is possible with the current and steady improvements of technology in digital imaging and solid state lasers. However, consistent seeding of such large and quiescent flow fields remains a challenge to be overcome.

PIV provides the velocity field measurement necessary for the doorway mass flow rate computation. However, the gas density field is also required. A vertical profile of gas density on centerline proved to be sufficient for the fluid analog experiment. The shortcomings of this method when applied in real fires should be quantified, especially in the high temperature outflow where the flow will be thermally stratified. 


\section{Reference List}

[1] J. Prahl and H. W. Emmons, Fire Induced Flow Through An Opening, Combustion and Flame 25 (3), 369-385 (1975).

[2] J. A. Rockett, Fire Induced Gas-Flow in An Enclosure, Combustion Science and Technology 12 (4-6), 165-175 (1976).

[3] B. J. McCaffrey and J. A. Rockett, Static Pressure Measurements of Enclosure Fires, Journal of Research of the National Bureau of Standards 82 (2), 107-117 (1977).

[4] K. Steckler, J. G. Quintiere, and W. J. Rinkinen, Flow Induced by Fire in a Compartment, Proceedings of the Combustion Institute 19 913-920 (1982).

[5] I. Nakaya, T. Tanaka, M. Yoshida, and K. Steckler, Doorway Flow Induced by A Propane Fire, Fire Safety Journal 10 (3), 185-195 (1986).

[6] H. W. Emmons, Vent Flows, The SFPE Handbook of Fire Protection Engineering, 3rd Edition (Section 2, Chap 3), National Fire Protection Association and The Society of Fire Protection Engineers, Quincy, MA, P. J. DiNenno, D. Drysdale, C. L. Beyler, and W. D. Walton, eds., 32-41 (2002).

[7] B. J. McCaffrey and G. Heskestad, Robust Bidirectional Low-Velocity Probe for Flame and Fire Application, Combustion and Flame 26 (1), 125-127 (1976).

[8] A. C. M. Braga, A Helium-Air Model for the Simulation of Fire Induced Flows, Worcester Polytechnic Institute, Worcester, MA, (1989).

[9] K. Steckler, J. G. Quintiere, and W. J. Rinkinen, Flow Induced by Fire in a Compartment, NBSIR 82-2520 National Bureau of Standards, Gaithersburg, MD, (1982).

[10] K. Kawagoe, Fire Behavior in Rooms, BRI Report 27 Building Research Institute, Ibaraki-Ken, Japan, (1958).

[11] R. J. Adrian, Particle-Imaging Techniques for Experimental Fluid-Mechanics, Annual Review of Fluid Mechanics 23 261-304 (1991).

[12] R. D. Keane and R. J. Adrian, Theory of Cross-Correlation Analysis of Piv Images, Applied Scientific Research 49 (3), 191-215 (1992).

[13] K. Steckler, J. G. Quintiere, and H. R. Baum, Fire Induced Flows Through Room Openings: Flow Coefficients, NBSIR 83-2801 National Bureau of Standards, Gaithersburg, MD, (1984). 
[14] J. G. Quintiere, Scaling Applications in Fire Research, Fire Safety Journal 15 (1), 3-29 (1989).

[15] K. Steckler, H. R. Baum, and J. G. Quintiere, Salt Water Modeling of Fire Induced Flows in Multicompartment Enclosures, Proceedings of the Combustion Institute 21 143-149 (1986).

[16] A. Hamins, K. Konishi, P. Borthwick, and T. Kashiwagi, Global Properties of Gaseous Pool Fires, Proceedings of the Combustion Institute 26 (1), 1429-1436 (1996).

[17] Guide to the Expression of Uncertainty in Measurement, International Organization for Standardization, Switzerland, (1995).

[18] C. E. Kuyatt and B. N. Taylor, Guidelines for Evaluating and Expressing the Uncertainty of NIST Measurement Results, NIST/TN-1297 NIST, Gaithersburg, MD, (1994).

[19] K. Steckler, J. G. Quintiere, and H. R. Baum, Fire Induced Flows Through Room Openings: Flow Coefficients, Proceedings of the Combustion Institute 20 15911600 (1984). 


\section{Appendix}

\section{.1 Tabulated values of the velocity and density field at the doorway plane}

Table 1 Tabulated values of $v_{x}$ velocity component $(\mathrm{m} / \mathrm{s})$ and density $\left(\mathrm{kg} / \mathrm{m}^{3}\right)$ at doorway plane, $x=-14 \mathrm{~mm}$.

\begin{tabular}{|c|c|c|c|c|c|c|}
\hline$y, \mathrm{~mm}$ & & & & & & $\mathrm{~mm}$ \\
\hline$y$, пит & -250.0 & -200.0 & -150.0 & -100.0 & -50.0 & 0.0 \\
\hline 39.7 & 0.000 & 0.000 & 0.000 & 0.000 & 0.000 & 0.000 \\
\hline 50.4 & 0.122 & 0.192 & 0.143 & 0.194 & 0.176 & 0.205 \\
\hline 61.1 & 0.159 & 0.214 & 0.212 & 0.234 & 0.209 & 0.240 \\
\hline 71.8 & 0.169 & 0.232 & 0.229 & 0.247 & 0.230 & 0.255 \\
\hline 82.5 & 0.183 & 0.239 & 0.240 & 0.255 & 0.243 & 0.266 \\
\hline 93.2 & 0.183 & 0.241 & 0.254 & 0.257 & 0.247 & 0.273 \\
\hline 103.9 & 0.179 & 0.239 & 0.251 & 0.256 & 0.245 & 0.274 \\
\hline 114.6 & 0.179 & 0.245 & 0.233 & 0.254 & 0.243 & 0.275 \\
\hline 125.3 & 0.175 & 0.251 & 0.243 & 0.251 & 0.246 & 0.279 \\
\hline 136.0 & 0.170 & 0.247 & 0.266 & 0.247 & 0.251 & 0.279 \\
\hline 146.7 & 0.172 & 0.242 & 0.292 & 0.244 & 0.249 & 0.274 \\
\hline 157.4 & 0.168 & 0.242 & 0.305 & 0.242 & 0.246 & 0.272 \\
\hline 168.1 & 0.164 & 0.235 & 0.300 & 0.236 & 0.249 & 0.271 \\
\hline 178.8 & 0.157 & 0.235 & 0.286 & 0.235 & 0.248 & 0.269 \\
\hline 189.5 & 0.154 & 0.234 & 0.255 & 0.235 & 0.246 & 0.265 \\
\hline 200.2 & 0.156 & 0.229 & 0.217 & 0.236 & 0.241 & 0.262 \\
\hline 210.9 & 0.149 & 0.228 & 0.200 & 0.234 & 0.239 & 0.258 \\
\hline 221.6 & 0.146 & 0.227 & 0.190 & 0.234 & 0.236 & 0.253 \\
\hline 232.3 & 0.143 & 0.221 & 0.192 & 0.235 & 0.236 & 0.252 \\
\hline 243.0 & 0.138 & 0.217 & 0.181 & 0.229 & 0.230 & 0.253 \\
\hline 253.7 & 0.132 & 0.239 & 0.164 & 0.223 & 0.228 & 0.249 \\
\hline 264.4 & 0.126 & 0.230 & 0.152 & 0.215 & 0.223 & 0.248 \\
\hline 275.1 & 0.114 & 0.207 & 0.147 & 0.207 & 0.220 & 0.248 \\
\hline 285.8 & 0.107 & 0.188 & 0.123 & 0.213 & 0.215 & 0.239 \\
\hline 296.5 & 0.117 & 0.182 & 0.102 & 0.211 & 0.213 & 0.234 \\
\hline 307.2 & 0.120 & 0.172 & 0.121 & 0.211 & 0.203 & 0.230 \\
\hline 317.9 & 0.144 & 0.202 & 0.189 & 0.213 & 0.220 & 0.256 \\
\hline 328.6 & 0.133 & 0.200 & 0.195 & 0.215 & 0.216 & 0.248 \\
\hline 339.3 & 0.133 & 0.203 & 0.208 & 0.227 & 0.214 & 0.245 \\
\hline 350.0 & 0.133 & 0.199 & 0.213 & 0.227 & 0.200 & 0.239 \\
\hline 360.7 & 0.135 & 0.196 & 0.210 & 0.228 & 0.200 & 0.231 \\
\hline 371.4 & 0.130 & 0.190 & 0.203 & 0.225 & 0.205 & 0.228 \\
\hline 382.1 & 0.117 & 0.192 & 0.199 & 0.221 & 0.210 & 0.227 \\
\hline 392.8 & 0.119 & 0.194 & 0.202 & 0.225 & 0.210 & 0.217 \\
\hline 403.5 & 0.117 & 0.198 & 0.208 & 0.226 & 0.208 & 0.211 \\
\hline 414.2 & 0.111 & 0.196 & 0.213 & 0.226 & 0.209 & 0.210 \\
\hline 424.9 & 0.114 & 0.191 & 0.210 & 0.221 & 0.207 & 0.214 \\
\hline 435.6 & 0.117 & 0.188 & 0.207 & 0.220 & 0.205 & 0.216 \\
\hline 446.3 & 0.118 & 0.187 & 0.205 & 0.219 & 0.201 & 0.214 \\
\hline 457.0 & 0.107 & 0.190 & 0.204 & 0.215 & 0.200 & 0.212 \\
\hline 467.7 & 0.095 & 0.197 & 0.206 & 0.216 & 0.198 & 0.212 \\
\hline 478.4 & 0.101 & 0.196 & 0.207 & 0.222 & 0.194 & 0.210 \\
\hline 489.1 & 0.108 & 0.196 & 0.204 & 0.218 & 0.194 & 0.207 \\
\hline 499.8 & 0.101 & 0.193 & 0.201 & 0.210 & 0.192 & 0.204 \\
\hline 510.5 & 0.096 & 0.198 & 0.208 & 0.213 & 0.199 & 0.212 \\
\hline 521.2 & 0.081 & 0.166 & 0.176 & 0.183 & 0.172 & 0.183 \\
\hline 531.9 & 0.091 & 0.199 & 0.212 & 0.234 & 0.217 & 0.220 \\
\hline 542.6 & 0.086 & 0.197 & 0.210 & 0.225 & 0.212 & 0.219 \\
\hline 553.3 & 0.085 & 0.205 & 0.211 & 0.218 & 0.204 & 0.219 \\
\hline 564.0 & 0.086 & 0.210 & 0.209 & 0.213 & 0.201 & 0.217 \\
\hline 574.7 & 0.082 & 0.197 & 0.200 & 0.204 & 0.198 & 0.214 \\
\hline 585.4 & 0.071 & 0.173 & 0.193 & 0.200 & 0.194 & 0.213 \\
\hline 596.1 & 0.061 & 0.168 & 0.189 & 0.201 & 0.192 & 0.213 \\
\hline 606.8 & 0.080 & 0.167 & 0.169 & 0.181 & 0.175 & 0.200 \\
\hline 617.5 & 0.101 & 0.197 & 0.194 & 0.205 & 0.200 & 0.229 \\
\hline 628.2 & 0.095 & 0.187 & 0.184 & 0.195 & 0.191 & 0.215 \\
\hline 638.9 & 0.095 & 0.176 & 0.177 & 0.191 & 0.190 & 0.211 \\
\hline 649.6 & 0.098 & 0.147 & 0.164 & 0.182 & 0.188 & 0.208 \\
\hline 660.3 & 0.098 & 0.119 & 0.147 & 0.170 & 0.183 & 0.203 \\
\hline 671.0 & 0.092 & 0.097 & 0.127 & 0.150 & 0.172 & 0.186 \\
\hline 681.7 & 0.089 & 0.073 & 0.102 & 0.127 & 0.156 & 0.162 \\
\hline 692.4 & 0.083 & 0.047 & 0.077 & 0.102 & 0.136 & 0.137 \\
\hline 703.1 & 0.076 & 0.019 & 0.043 & 0.070 & 0.101 & 0.097 \\
\hline 713.8 & 0.063 & -0.027 & -0.001 & 0.030 & 0.052 & 0.043 \\
\hline 724.5 & 0.039 & -0.076 & -0.052 & -0.024 & 0.002 & -0.013 \\
\hline 735.2 & 0.019 & -0.123 & -0.107 & -0.076 & -0.055 & -0.075 \\
\hline 745.9 & 0.006 & -0.171 & -0.160 & -0.127 & -0.127 & -0.137 \\
\hline 756.6 & -0.016 & -0.217 & -0.205 & -0.187 & -0.201 & -0.199 \\
\hline 767.3 & -0.045 & -0.265 & -0.247 & -0.253 & -0.271 & -0.261 \\
\hline 778.0 & -0.057 & -0.325 & -0.295 & -0.305 & -0.330 & -0.319 \\
\hline 788.7 & -0.050 & -0.373 & -0.338 & -0.341 & -0.376 & -0.374 \\
\hline 799.4 & -0.041 & $\begin{array}{l}-0.392 \\
\end{array}$ & -0.384 & -0.374 & -0.408 & -0.419 \\
\hline 810.1 & -0.034 & -0.395 & -0.420 & -0.411 & -0.437 & -0.455 \\
\hline 820.8 & -0.009 & -0.367 & -0.434 & -0.437 & -0.454 & -0.483 \\
\hline 831.5 & 0.019 & -0.351 & -0.470 & -0.475 & -0.495 & -0.524 \\
\hline 842.2 & 0.015 & -0.282 & -0.417 & -0.421 & -0.434 & -0.456 \\
\hline 852.9 & -0.024 & -0.279 & -0.575 & -0.577 & -0.565 & -0.515 \\
\hline 863.6 & -0.049 & -0.251 & -0.590 & -0.595 & -0.587 & -0.420 \\
\hline 874.3 & -0.036 & -0.239 & -0.582 & -0.610 & -0.603 & -0.369 \\
\hline 885.0 & 0.018 & -0.213 & -0.595 & -0.630 & -0.623 & -0.383 \\
\hline 895.7 & 0.034 & -0.218 & -0.613 & -0.656 & -0.646 & -0.435 \\
\hline 906.4 & 0.022 & -0.265 & -0.609 & -0.675 & -0.667 & -0.469 \\
\hline 917.1 & 0.017 & -0.297 & -0.613 & -0.694 & -0.691 & -0.469 \\
\hline 927.8 & -0.001 & -0.326 & -0.610 & -0.704 & -0.712 & -0.384 \\
\hline 938.5 & -0.044 & -0.375 & -0.622 & -0.692 & -0.716 & -0.348 \\
\hline 949.2 & -0.094 & -0.460 & -0.643 & -0.690 & -0.713 & -0.552 \\
\hline 959.9 & -0.113 & -0.534 & -0.645 & -0.688 & -0.713 & -0.739 \\
\hline 970.6 & -0.089 & -0.469 & -0.610 & -0.647 & -0.689 & -0.727 \\
\hline 981.3 & -0.079 & -0.384 & -0.545 & -0.578 & -0.632 & -0.603 \\
\hline 992.0 & -0.113 & -0.447 & -0.506 & -0.549 & -0.592 & -0.412 \\
\hline
\end{tabular}




\begin{tabular}{|c|c|c|c|c|c|c|c|c|c|c|c|c|}
\hline 1002.7 & $\begin{array}{c}-0.148 \\
\end{array}$ & $\begin{array}{l}-0.498 \\
\end{array}$ & $\begin{array}{l}-0.526 \\
\end{array}$ & -0.522 & -0.559 & $\begin{array}{l}-0.306 \\
\end{array}$ & $\begin{array}{l}-0.126 \\
\end{array}$ & $\begin{array}{l}-0.471 \\
\end{array}$ & -0.653 & -0.950 & $\begin{array}{c}-0.167 \\
\end{array}$ & $\overline{c 0.977}$ \\
\hline 1013.4 & -0.130 & -0.482 & -0.558 & -0.469 & -0.427 & -0.283 & -0.082 & -0.477 & -0.534 & -0.906 & -0.147 & 0.977 \\
\hline 1024.1 & -0.069 & -0.308 & -0.424 & -0.287 & -0.207 & -0.173 & -0.063 & -0.394 & -0.306 & -0.596 & -0.093 & 0.977 \\
\hline 1034.8 & -0.028 & -0.100 & -0.151 & -0.015 & -0.092 & -0.063 & -0.040 & -0.191 & -0.134 & -0.198 & -0.047 & 0.977 \\
\hline 1045.5 & 0.000 & 0.000 & 0.000 & 0.000 & 0.000 & 0.000 & 0.000 & 0.000 & 0.000 & 0.000 & 0.000 & 0.977 \\
\hline
\end{tabular}

Table 2 Tabulated values of $v_{y}$ velocity component $(\mathrm{m} / \mathrm{s})$ at doorway plane, $x=-14$ mm.

\begin{tabular}{|c|c|}
\hline$y, \mathrm{~mm}$ & \\
\hline$y, \ln$ & -250.0 \\
\hline 39.7 & 0.000 \\
\hline 50.4 & 0.022 \\
\hline 61.1 & 0.026 \\
\hline 71.8 & 0.024 \\
\hline 82.5 & 0.016 \\
\hline 93.2 & 0.014 \\
\hline 103.9 & 0.017 \\
\hline 114.6 & 0.015 \\
\hline 125.3 & 0.013 \\
\hline 136.0 & 0.009 \\
\hline 146.7 & 0.006 \\
\hline 157.4 & 0.006 \\
\hline 168.1 & 0.007 \\
\hline 178.8 & 0.008 \\
\hline 189.5 & 0.009 \\
\hline 200.2 & 0.008 \\
\hline 210.9 & 0.005 \\
\hline 221.6 & 0.001 \\
\hline 232.3 & 0.002 \\
\hline 243.0 & 0.007 \\
\hline 253.7 & 0.011 \\
\hline 264.4 & 0.005 \\
\hline 275.1 & 0.000 \\
\hline 285.8 & -0.004 \\
\hline 296.5 & -0.005 \\
\hline 307.2 & -0.012 \\
\hline 317.9 & -0.017 \\
\hline 328.6 & -0.019 \\
\hline 339.3 & -0.016 \\
\hline 350.0 & -0.019 \\
\hline 360.7 & -0.023 \\
\hline 371.4 & -0.025 \\
\hline 382.1 & -0.024 \\
\hline 392.8 & -0.022 \\
\hline 403.5 & -0.021 \\
\hline 414.2 & -0.024 \\
\hline 424.9 & $\begin{array}{l}-0.024 \\
-0.025\end{array}$ \\
\hline 435.6 & -0.027 \\
\hline 446.3 & -0.026 \\
\hline 457.0 & -0.026 \\
\hline 467.7 & $\begin{array}{l}-0.026 \\
\end{array}$ \\
\hline 478.4 & -0.025 \\
\hline 489.1 & -0.021 \\
\hline 499.8 & -0.013 \\
\hline 510.5 & -0.015 \\
\hline 521.2 & -0.017 \\
\hline 531.9 & -0.022 \\
\hline 542.6 & -0.025 \\
\hline 553.3 & -0.029 \\
\hline 564.0 & -0.033 \\
\hline 574.7 & -0.037 \\
\hline 585.4 & -0.038 \\
\hline 596.1 & -0.034 \\
\hline 606.8 & -0.035 \\
\hline 617.5 & -0.044 \\
\hline 628.2 & $\begin{array}{r}-0.0448 \\
-0.048\end{array}$ \\
\hline 638.9 & -0.054 \\
\hline 649.6 & -0.060 \\
\hline 660.3 & -0.061 \\
\hline 671.0 & -0.056 \\
\hline 681.7 & -0.056 \\
\hline 692.4 & -0.048 \\
\hline 703.1 & -0.031 \\
\hline 713.8 & -0.010 \\
\hline 724.5 & 0.014 \\
\hline 735.2 & 0.042 \\
\hline 745.9 & 0.063 \\
\hline 756.6 & 0.079 \\
\hline 767.3 & 0.096 \\
\hline 778.0 & 0.106 \\
\hline 788.7 & 0.106 \\
\hline 799.4 & 0.106 \\
\hline 810.1 & 0.112 \\
\hline 820.8 & 0.113 \\
\hline 831.5 & 0.119 \\
\hline 842.2 & 0.109 \\
\hline 852.9 & 0.143 \\
\hline 863.6 & 0.131 \\
\hline 874.3 & 0.101 \\
\hline 885.0 & 0.066 \\
\hline 895.7 & 0.040 \\
\hline 906.4 & 0.015 \\
\hline 917.1 & 0.020 \\
\hline 927.8 & 0.041 \\
\hline 938.5 & 0.019 \\
\hline 949.2 & -0.001 \\
\hline 959.9 & -0.001 \\
\hline 970.6 & -0.004 \\
\hline 981.3 & $\begin{array}{l}-0.011 \\
\end{array}$ \\
\hline 992.0 & -0.029 \\
\hline 1002.7 & -0.038 \\
\hline 1013.4 & -0.026 \\
\hline 1024.1 & 0.001 \\
\hline 1034.8 & 0.006 \\
\hline 1045.5 & 0.000 \\
\hline
\end{tabular}


Table 3 Tabulated values of $v_{z}$ velocity component $(\mathrm{m} / \mathrm{s})$ at doorway plane, $x=-14$ mm.

\begin{tabular}{|c|c|}
\hline$y, \mathrm{~mm}$ & \\
\hline$y, 1 \mathrm{~m}$ & -250.0 \\
\hline 39.7 & 0.000 \\
\hline 50.4 & -0.051 \\
\hline 61.1 & -0.013 \\
\hline 71.8 & -0.001 \\
\hline 82.5 & 0.013 \\
\hline 93.2 & 0.023 \\
\hline 103.9 & 0.024 \\
\hline 114.6 & 0.025 \\
\hline 125.3 & 0.023 \\
\hline 136.0 & 0.022 \\
\hline 146.7 & 0.023 \\
\hline 157.4 & 0.029 \\
\hline 168.1 & 0.037 \\
\hline 178.8 & 0.038 \\
\hline 189.5 & 0.038 \\
\hline 200.2 & 0.035 \\
\hline 210.9 & 0.034 \\
\hline 221.6 & 0.036 \\
\hline 232.3 & 0.038 \\
\hline 243.0 & 0.034 \\
\hline 253.7 & 0.034 \\
\hline 264.4 & 0.033 \\
\hline 275.1 & 0.038 \\
\hline 285.8 & 0.037 \\
\hline 296.5 & 0.037 \\
\hline 307.2 & 0.036 \\
\hline 317.9 & 0.081 \\
\hline 328.6 & 0.081 \\
\hline 339.3 & 0.081 \\
\hline 350.0 & 0.078 \\
\hline 360.7 & 0.075 \\
\hline 371.4 & 0.082 \\
\hline 382.1 & 0.090 \\
\hline 392.8 & 0.090 \\
\hline 403.5 & 0.093 \\
\hline 414.2 & 0.087 \\
\hline 424.9 & 0.085 \\
\hline 435.6 & 0.080 \\
\hline 446.3 & 0.077 \\
\hline 457.0 & 0.070 \\
\hline 467.7 & 0.066 \\
\hline 478.4 & 0.069 \\
\hline 489.1 & 0.073 \\
\hline 499.8 & 0.077 \\
\hline 510.5 & 0.078 \\
\hline 521.2 & 0.062 \\
\hline 531.9 & 0.073 \\
\hline 542.6 & 0.070 \\
\hline 553.3 & 0.071 \\
\hline 564.0 & 0.075 \\
\hline 574.7 & 0.075 \\
\hline 585.4 & 0.075 \\
\hline 596.1 & 0.075 \\
\hline 606.8 & 0.041 \\
\hline 617.5 & 0.036 \\
\hline 628.2 & 0.030 \\
\hline 638.9 & 0.020 \\
\hline 649.6 & 0.011 \\
\hline 660.3 & 0.008 \\
\hline 671.0 & 0.003 \\
\hline 681.7 & -0.005 \\
\hline 692.4 & -0.003 \\
\hline 703.1 & -0.001 \\
\hline 713.8 & -0.001 \\
\hline 724.5 & -0.001 \\
\hline 735.2 & -0.002 \\
\hline 745.9 & 0.003 \\
\hline 756.6 & 0.015 \\
\hline 767.3 & 0.029 \\
\hline 778.0 & 0.040 \\
\hline 788.7 & 0.046 \\
\hline 799.4 & 0.050 \\
\hline 810.1 & 0.054 \\
\hline 820.8 & 0.047 \\
\hline 831.5 & 0.047 \\
\hline 842.2 & 0.049 \\
\hline 852.9 & 0.119 \\
\hline 863.6 & 0.155 \\
\hline 874.3 & 0.158 \\
\hline 885.0 & 0.130 \\
\hline 895.7 & 0.103 \\
\hline 906.4 & 0.093 \\
\hline 917.1 & 0.079 \\
\hline 927.8 & 0.072 \\
\hline 938.5 & 0.065 \\
\hline 949.2 & 0.069 \\
\hline 959.9 & 0.063 \\
\hline 970.6 & 0.045 \\
\hline 981.3 & 0.030 \\
\hline 992.0 & 0.013 \\
\hline 1002.7 & $\begin{array}{l}-0.022 \\
-\end{array}$ \\
\hline 1013.4 & -0.044 \\
\hline 1024.1 & -0.047 \\
\hline 1034.8 & -0.032 \\
\hline 1045.5 & 0.000 \\
\hline
\end{tabular}


Table 4 Tabulated values of $|\mathrm{V}|(\mathrm{m} / \mathrm{s})$ at doorway plane, $x=-14 \mathrm{~mm}$.

\begin{tabular}{|c|c|}
\hline$y, \mathrm{~mm}$ & \\
\hline & -250.0 \\
\hline 39.7 & 0.000 \\
\hline 50.4 & 0.134 \\
\hline 61.1 & 0.162 \\
\hline 71.8 & 0.171 \\
\hline 82.5 & 0.184 \\
\hline 93.2 & 0.185 \\
\hline 103.9 & 0.181 \\
\hline 114.6 & 0.181 \\
\hline 125.3 & 0.177 \\
\hline 136.0 & 0.172 \\
\hline 146.7 & 0.173 \\
\hline 157.4 & 0.171 \\
\hline 168.1 & 0.168 \\
\hline 178.8 & 0.161 \\
\hline 189.5 & 0.159 \\
\hline 200.2 & 0.160 \\
\hline 210.9 & 0.153 \\
\hline 221.6 & 0.150 \\
\hline 232.3 & 0.148 \\
\hline 243.0 & 0.142 \\
\hline 253.7 & 0.137 \\
\hline 264.4 & 0.130 \\
\hline 275.1 & 0.121 \\
\hline 285.8 & 0.113 \\
\hline 296.5 & 0.122 \\
\hline 307.2 & 0.126 \\
\hline 317.9 & 0.166 \\
\hline 328.6 & 0.156 \\
\hline 339.3 & 0.157 \\
\hline 350.0 & 0.156 \\
\hline 360.7 & 0.156 \\
\hline 371.4 & 0.156 \\
\hline 382.1 & 0.149 \\
\hline 392.8 & 0.151 \\
\hline 403.5 & 0.151 \\
\hline 414.2 & 0.143 \\
\hline 424.9 & 0.144 \\
\hline 435.6 & 0.144 \\
\hline 446.3 & 0.143 \\
\hline 457.0 & 0.131 \\
\hline 467.7 & 0.119 \\
\hline 478.4 & 0.125 \\
\hline 489.1 & 0.132 \\
\hline 499.8 & 0.128 \\
\hline 510.5 & 0.125 \\
\hline 521.2 & 0.103 \\
\hline 531.9 & 0.118 \\
\hline 542.6 & 0.114 \\
\hline 553.3 & 0.114 \\
\hline 564.0 & 0.119 \\
\hline 574.7 & 0.117 \\
\hline 585.4 & 0.110 \\
\hline 596.1 & 0.103 \\
\hline 606.8 & 0.097 \\
\hline 617.5 & 0.116 \\
\hline 628.2 & 0.111 \\
\hline 638.9 & 0.111 \\
\hline 649.6 & 0.116 \\
\hline 660.3 & 0.116 \\
\hline 671.0 & 0.108 \\
\hline 681.7 & 0.106 \\
\hline 692.4 & 0.096 \\
\hline 703.1 & 0.082 \\
\hline 713.8 & 0.063 \\
\hline 724.5 & 0.042 \\
\hline 735.2 & 0.046 \\
\hline 745.9 & 0.064 \\
\hline 756.6 & $\begin{array}{r}-.004 \\
-0.082\end{array}$ \\
\hline 767.3 & -0.110 \\
\hline 778.0 & -0.126 \\
\hline 788.7 & -0.126 \\
\hline 799.4 & -0.124 \\
\hline 810.1 & -0.129 \\
\hline 820.8 & -0.123 \\
\hline 831.5 & 0.130 \\
\hline 842.2 & 0.120 \\
\hline 852.9 & -0.187 \\
\hline 863.6 & $\begin{array}{r}-0.101 \\
-0.209\end{array}$ \\
\hline 874.3 & -0.190 \\
\hline 885.0 & 0.147 \\
\hline 895.7 & 0.116 \\
\hline 906.4 & 0.096 \\
\hline 917.1 & 0.083 \\
\hline 927.8 & -0.083 \\
\hline 938.5 & -0.081 \\
\hline 949.2 & -0.116 \\
\hline 959.9 & -0.129 \\
\hline 970.6 & -0.100 \\
\hline 981.3 & -0.085 \\
\hline 992.0 & -0.117 \\
\hline 1002.7 & -0.154 \\
\hline 1013.4 & -0.140 \\
\hline 1024.1 & -0.083 \\
\hline 1034.8 & -0.043 \\
\hline 1045.5 & 0.000 \\
\hline
\end{tabular}




\section{$.2 \quad$ Numerical Integration}

A numerical integration of the mass flow rate at the doorway was computed using the Trapezoid Rule.

$$
\int_{a}^{b} f(x) d x=c\left(\frac{1}{2} f_{0}+f_{1}+f_{2}+\ldots .+f_{n-1}+\frac{1}{2} f_{n}\right), \quad \text { where } \quad c=(b-a) / n
$$

Mass flow rate was computed for the inflow and outflow separately and in each case the computation was performed with two steps worth describing. First a numerical integration was performed along a vertical line to compute a line mass flux at each $\mathrm{z}$ measurement location. For the inflow, the lower limit of the vertical integration, a, was $39.7 \mathrm{~mm}$. This is the location of the door sill and here velocity was forced to zero. The upper limit of integration, b, was defined by the local neutral plane. The vertical measurement spacing was $10.7 \mathrm{~mm}$, which was less than the spatial resolution of the measurement $(21.4 \mathrm{~mm})$ due to the $50 \%$ overlap in the cross correlation of vectors. Therefore assigning the location of the final positive $v_{x}$ velocity as the neutral plane location is sufficient. The velocity values are approximately zero, therefore interpolating to find the exact zero location would be of negligible benefit. For example, at $\mathrm{z}=0.0$ $\mathrm{mm}$, the last positive value is $0.043 \mathrm{~m} / \mathrm{s}$ and occurs at $713.8 \mathrm{~mm}$. This location is selected as the upper limit of integration, b, instead of some location between $713.8 \mathrm{~mm}$ and $724.5 \mathrm{~mm}$. This first step of the mass flow rate computation is demonstrated in Table 5 , where the line mass flux of the flow into the enclosure (shaded regions) is computed.

Table 5 Step 1 of the numerical integration to compute mass flow rate into the enclosure (shaded regions): Mass flux along a vertical line.

\begin{tabular}{|c|c|c|c|c|c|c|c|c|c|c|c|c|}
\hline$y, \mathrm{~mm}$ & -250.0 & -200.0 & -150.0 & -100.0 & -50.0 & $\begin{array}{r}\mathrm{z}, \mathrm{mm} \\
0.0 \\
\end{array}$ & 50.0 & 100.0 & 150.0 & 200.0 & 250.0 & $\begin{array}{l}\rho, \\
\mathrm{kg} / \mathrm{m}^{3}\end{array}$ \\
\hline \multicolumn{13}{|c|}{$v_{x}, \mathrm{~m} / \mathrm{s}$} \\
\hline 39.7 & 0.000 & 0.000 & 0.000 & 0.000 & 0.000 & 0.000 & 0.000 & 0.000 & 0.000 & 0.000 & 0.000 & 1.172 \\
\hline 50.4 & 0.122 & 0.192 & 0.143 & 0.194 & 0.176 & 0.205 & 0.186 & 0.200 & 0.201 & 0.175 & 0.155 & 1.172 \\
\hline 61.1 & 0.159 & 0.214 & 0.212 & 0.234 & 0.209 & 0.240 & 0.217 & 0.222 & 0.245 & 0.200 & 0.171 & 1.172 \\
\hline 71.8 & 0.169 & 0.232 & 0.229 & 0.247 & 0.230 & 0.255 & 0.236 & 0.240 & 0.266 & 0.216 & 0.170 & 1.172 \\
\hline 82.5 & 0.183 & 0.239 & 0.240 & 0.255 & 0.243 & 0.266 & 0.251 & 0.249 & 0.271 & 0.225 & 0.166 & 1.172 \\
\hline 93.2 & 0.183 & 0.241 & 0.254 & 0.257 & 0.247 & 0.273 & 0.258 & 0.251 & 0.268 & 0.230 & 0.167 & 1.172 \\
\hline 103.9 & 0.179 & 0.239 & 0.251 & 0.256 & 0.245 & 0.274 & 0.261 & 0.250 & 0.268 & 0.227 & 0.171 & 1.172 \\
\hline .. & .. & .. & .. & .. & .. & .. & .. & .. & .. & .. & .. & .. \\
\hline .. & .. & .. & .. & .. & .. & .. & .. & .. & .. & .. & .. & .. \\
\hline .. & & .. & .. & .. & .. & .. & .. & .. & .. & 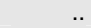 & .. & .. \\
\hline 692.4 & 0.083 & 0.047 & 0.077 & 0.102 & 0.136 & 0.137 & 0.131 & 0.068 & 0.144 & 0.129 & 0.069 & 1.110 \\
\hline 703.1 & 0.076 & 0.019 & 0.043 & 0.070 & 0.101 & 0.097 & 0.106 & 0.026 & 0.132 & 0.119 & 0.065 & 1.080 \\
\hline 713.8 & 0.063 & -0.027 & -0.001 & 0.030 & 0.052 & 0.043 & 0.078 & -0.017 & 0.115 & 0.106 & 0.058 & 1.059 \\
\hline 724.5 & 0.039 & -0.076 & -0.052 & -0.024 & 0.002 & -0.013 & 0.034 & -0.066 & 0.087 & 0.080 & 0.044 & 1.047 \\
\hline 735.2 & 0.019 & -0.123 & -0.107 & -0.076 & -0.055 & -0.075 & -0.011 & -0.123 & 0.051 & 0.049 & 0.032 & 1.034 \\
\hline 745.9 & 0.006 & -0.171 & -0.160 & -0.127 & -0.127 & -0.137 & -0.064 & -0.181 & 0.005 & 0.017 & 0.023 & 1.019 \\
\hline 756.6 & -0.016 & -0.217 & -0.205 & -0.187 & -0.201 & -0.199 & -0.126 & -0.235 & -0.045 & -0.024 & 0.008 & 1.013 \\
\hline 767.3 & -0.045 & -0.265 & -0.247 & -0.253 & -0.271 & -0.261 & -0.186 & -0.282 & -0.104 & -0.074 & -0.011 & 1.009 \\
\hline 778.0 & -0.057 & -0.325 & -0.295 & -0.305 & -0.330 & -0.319 & -0.252 & -0.324 & -0.164 & -0.130 & -0.034 & 1.006 \\
\hline \\
\hline$\rho v_{x} d y$ & 0.096 & 0.151 & 0.152 & 0.166 & 0.163 & 0.178 & 0.166 & 0.159 & 0.168 & 0.136 & 0.097 & \\
\hline
\end{tabular}


The second step of the mass flow rate computation was a numerical integration across the width of the doorway, therefore integrating the line mass fluxes. The numerical integration routine required a constant spacing $(\mathrm{dx})$, therefore in order to include the solid boundaries which occurred at $-270 \mathrm{~mm}$ and $270 \mathrm{~mm}$ the line mass fluxes computed at 50 $\mathrm{mm}$ intervals were used to interpolate values at $10 \mathrm{~mm}$ intervals. The numerical integration across the width of the doorway to complete the mass flow rate computation is demonstrated in Table 6.

Table 6 Step 2 of the numerical integration to compute mass flow rate into the enclosure: Integration across the width of the doorway of the interpolated line mass fluxes.

\begin{tabular}{ccccccccc}
\hline \hline $\boldsymbol{z}, \mathbf{m m}$ & $\mathbf{- 2 7 0 . 0}$ & $\mathbf{- 2 6 0 . 0}$ & $\mathbf{- 2 5 0 . 0}$ &.. &.. & $\mathbf{2 5 0 . 0}$ & $\mathbf{2 6 0 . 0}$ & $\mathbf{2 7 0 . 0}$ \\
\hline$\left[\int_{y_{0}}^{h_{N}} \rho v_{x} d y\right]_{\text {Interpolated }}$ & 0.000 & 0.048 & 0.096 &.. &.. & 0.097 & 0.049 & 0.000 \\
& $\dot{m}_{\text {in,exp }}=\int_{-W / 2}^{W / 2}\left[\int_{y_{0}}^{h_{N}} \rho v_{x} d y\right]_{\text {Interpolated }} d z=0.079 \mathrm{~kg} / \mathrm{s}$ & & \\
\hline
\end{tabular}

The same process is followed to compute mass flow rate out of the enclosure. In this case the lower limit of integration, a, is variable and is defined by the location of the first negative velocity value. The upper limit, b, is the door soffit, located at $1045.5 \mathrm{~mm}$. Velocity at this boundary is forced to zero. 